Supprorting Information for:

\title{
A Tandem Semipinacol/Schmidt Reaction Leading to Versatile and Efficient Approach to Azaquaternary Alkaloid Skeletons
}

\author{
Peiming Gu, Yu-Ming Zhao, Yong Qiang Tu,* Yufei Ma, and Fumin Zhang
}

State Key Laboratory of Applied Organic Chemistry and Department of Chemistry, Lanzhou

University, Lanzhou 730000, P R China

E-mail: tuyq@lzu.edu.cn

Table of Contents:

General Procedure for the Synthesis Substrates 1a-1g and Spectral Data

Procedure for the Synthesis Substrates $1 \mathrm{i}$ and $1 \mathrm{j}$ and Spectral Data

Procedure for the Synthesis Substrates 1i and 1j and Spectral Data

Procedure for the Tandem Semipinacol/intramolecular Aube's type Schmidt reaction

Spectral Data for Tandem Products

Synthesis of azaquaternary 5-7-5 ring skeleton from $2 \mathrm{~h}$ 
Note: ${ }^{1} \mathrm{H}$ and ${ }^{13} \mathrm{C}$ NMR spectra were recorded in $\mathrm{CDCl}_{3}$ solution on Varian Mercury-300 or $400 \mathrm{MHz}$. The MS data were obtained with EI $(70 \mathrm{eV})$. High-resolution mass spectral analysis (HRMS) data were measured on the Bruker ApexII by means of the ESI technique.

\section{General Procedure for the Synthesis Substrates 1a-1g and Spectral Data}<smiles>C=C(Br)CCCOCCCC(=C)Br</smiles><smiles>[R]C([R])(O)C(=C)CCCO</smiles>

5

1. $\mathrm{MsCl}, \mathrm{Et}_{3} \mathrm{~N}$

2. $\mathrm{NaN}_{3}, \mathrm{DMSO}$

3. TMSCl, imidazole, DMF

4. $\mathrm{m}-\mathrm{CPBA}, \mathrm{NaHCO} 3$

Methods A

1. $\mathrm{MsCl}, \mathrm{Et}_{3} \mathrm{~N}$

2. TMSCl, HMDS, pyridine

5
3. $\mathrm{NaN}_{3}$, DMF

4. $\mathrm{m}-\mathrm{CPBA}, \mathrm{NaHCO} 3$<smiles>[R]C([R])(OS(C)(=O)=O)C1(CCCN)CO1</smiles>

1<smiles>[R]C([R])(OS(C)(=O)=O)C1(CCCN)CO1</smiles>

1

All these substrates were synthesized by normal chemical transformations. Generally vinyl lithium with a transferable function was added to a ketone. Then the transferable function was changed into azido and epoxided following protecting by TMS (Methods A). Alternatively the allylic alcohol was protected by TMS following change to azide, then it was epoxided (Methods B). A Typical Procedure for the Synthesis Substrate 1b was listed as a model (Methods A).<smiles>C=C(Br)CCC[OH+]</smiles>

A solution of known compound vinyl bromide $3^{1}(2.00 \mathrm{~g}, 12.1 \mathrm{mmol})$ in $100 \mathrm{ml}$ of DMF was treated with tert-butylchlorodimethylsilane (TBSCl, $2.22 \mathrm{~g}, 14.7 \mathrm{mmol})$, imidazole $(4.18 \mathrm{~g}, 61.5$ mmol) at room temperature. After $1 \mathrm{~h}$, the reaction mixture was poured into $400 \mathrm{~mL}$ ether and washed with water, dried over $\mathrm{Na}_{2} \mathrm{SO}_{4}$, filtered and concentrated. Flash chromatography on silica gel afforded $3.30 \mathrm{~g}$ of a new vinyl bromide $\mathbf{4}$ as a clear oil (98\% yield): ${ }^{1} \mathrm{H}$ NMR $\left(300 \mathrm{MHz}, \mathrm{CDCl}_{3}\right.$ ) $\delta 0.04(\mathrm{~s}, 6 \mathrm{H}), 0.89(\mathrm{~s}, 9 \mathrm{H}), 1.74 \sim 1.79(\mathrm{~m}, 2 \mathrm{H}), 2.50(\mathrm{t}, 2 \mathrm{H}), 3.63(\mathrm{t}, 2 \mathrm{H}), 5.40(\mathrm{~s}, 1 \mathrm{H}), 5.58(\mathrm{t}, 1 \mathrm{H})$; 
A Tandem Semipinacol/Schmidt Reaction Leading to Versatile and Efficient Approach to Azaquaternary Alkaloid Skeletons $\quad$ Peiming Gu, Yu-Ming Zhao, Yong Qiang Tu, ${ }^{*}$ Yufei Ma, and Fumin Zhang

${ }^{13} \mathrm{C}$ NMR $\left(75 \mathrm{MHz}, \mathrm{CDCl}_{3}\right) \delta-5.3,18.3,25.9,31.0,37.8,61.4,116.7,134.3 ; \mathrm{MS}$ (EI) m/e 265, $263,223,221,169,167,139,75,67$.

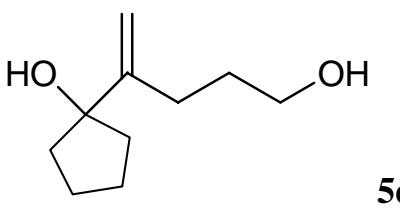

$5 e$

A solution of the vinyl bromide $4(2.1 \mathrm{~g}, 7.53 \mathrm{mmol})$ in $12 \mathrm{~mL}$ of THF was treated dropwise with n-butyllithium at $-78{ }^{\circ} \mathrm{C}(4.2 \mathrm{~mL}, 1.8 \mathrm{M}$ in hexane $7.56 \mathrm{mmol})$, followed by addition of cyclopentanone $(0.80 \mathrm{~mL}, 9.04 \mathrm{mmol})$ after $20 \mathrm{~min}$. The reaction mixture was allowed to warm to ambient temperature, quenched by addition of water $(5 \mathrm{~mL})$, extracted with ether $(2 \times 70 \mathrm{~mL})$, dried over anhydrous $\mathrm{MgSO}_{4}$, filtered and concentrated. The siloxyl ether was treated with tetrabutylammonium fluoride trihydrate $(10 \mathrm{~g}, 31.7 \mathrm{mmol})$ at room temperature. After $2 \mathrm{~h}$, ether $(200 \mathrm{~mL})$ was added, and the mixture was washed with water and brine and then dried $\left(\mathrm{Na}_{2} \mathrm{SO}_{4}\right)$ and concentrated. Chromatography gave $0.77 \mathrm{~g}$ of the desired compound $5 \mathbf{e}$ (60\% yield): ${ }^{1} \mathrm{H}$ NMR $\left(300 \mathrm{MHz}, \mathrm{CDCl}_{3}\right) \delta 1.66 \sim 1.91(\mathrm{~m}, 10 \mathrm{H}), 2.04(\mathrm{bs}, 2 \mathrm{H}), 2.22(\mathrm{t}, 2 \mathrm{H}, \mathrm{J}=7.5 \mathrm{~Hz}), 3.69(\mathrm{t}, 2 \mathrm{H}, \mathrm{J}=6 \mathrm{~Hz})$, 4.85(d, $1 \mathrm{H}, \mathrm{J}=0.9 \mathrm{~Hz}), 5.14(\mathrm{~s}, 1 \mathrm{H}) ;{ }^{13} \mathrm{C}$ NMR $\left(75 \mathrm{MHz}, \mathrm{CDCl}_{3}\right) \delta 23.2,27.6,31.6,38.4,61.9,84.5$, 107.8, 153.1; MS (EI) m/e 170, 152, 137, 125, 111, 85, 67, 55.<smiles>C=C(CCCN)C1(O)CCCC1</smiles>

Methanesulfonyl chloride $(0.55 \mathrm{~g}, 4.75 \mathrm{mmol})$ was added to a solution of compound $5 \mathbf{e}(0.77 \mathrm{~g}$, $4.53 \mathrm{mmoL})$ and triethylamine $(0.95 \mathrm{~mL}, 6.83 \mathrm{mmol})$ in $\mathrm{CH}_{2} \mathrm{Cl}_{2}$ at $0{ }^{\circ} \mathrm{C}$. After $10 \mathrm{~min}$, the mixture was diluted with $\mathrm{CH}_{2} \mathrm{Cl}_{2}$ and washed with brine, and then dried $\left(\mathrm{Na}_{2} \mathrm{SO}_{4}\right)$ and concentrated to give the crude mesylate, which was diluted in dimehyl sulfoxide (DMSO, $5 \mathrm{~mL}$ ) and treated with $\mathrm{NaN}_{3}$. The reaction mixture was stirred for $18 \mathrm{~h}$ and $25 \mathrm{~mL}$ of $\mathrm{H}_{2} \mathrm{O}$ was added. After dilution with ether $(150 \mathrm{~mL})$, the organic layer was separated, and washed with water, brine, dried over $\mathrm{Na}_{2} \mathrm{SO}_{4}$ and concentrated in vacuum, chromatographed to give a clear oil 6e (478 $\mathrm{mg}$, $84 \%$ yield): ${ }^{1} \mathrm{H}$ NMR $\left(300 \mathrm{MHz}, \mathrm{CDCl}_{3}\right) \delta 1.33(\mathrm{~s}, 1 \mathrm{H}), 1.63 \sim 1.89(\mathrm{~m}, 10 \mathrm{H}), 2.20(\mathrm{t}, 2 \mathrm{H}, \mathrm{J}=7.8 \mathrm{~Hz})$, $3.33(\mathrm{t}, 2 \mathrm{H}, \mathrm{J}=6.9 \mathrm{~Hz}), 4.82(\mathrm{~s}, 1 \mathrm{H}), 5.16(\mathrm{~s}, 1 \mathrm{H}) ;{ }^{13} \mathrm{C} \mathrm{NMR}\left(75 \mathrm{MHz}, \mathrm{CDCl}_{3}\right) \delta 23.4(2 \mathrm{C}), 28.0,28.7$, 38.7(2C), 51.2, 84.5, 108.4, 152.5; IR 2096.3, $1640.3 \mathrm{~cm}^{-1}$.

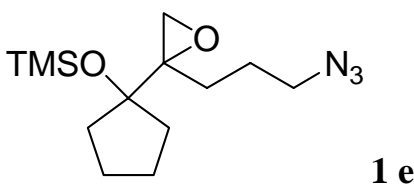

The azide 6e $(478 \mathrm{mg}, 2.45 \mathrm{mmol})$ was diluted into $\mathrm{CH}_{2} \mathrm{Cl}_{2}(25 \mathrm{~mL})$. $\mathrm{NaHCO}_{3}(247 \mathrm{mg}, 2.94$ $\mathrm{mmol}$ ) was added and then treated with 3-chloroperoxybenzoic acid (m-CPBA, $509 \mathrm{mg}, 2.94$ mmol). After $2.5 \mathrm{~h}$, the reaction mixture was diluted with $\mathrm{CH}_{2} \mathrm{Cl}_{2}$ and washed with saturated $\mathrm{K}_{2} \mathrm{CO}_{3}$ solution, dried over $\mathrm{Na}_{2} \mathrm{SO}_{4}$, concentrated. The crude product was diluted into DMF $(5 \mathrm{~mL})$ and treated trimethylchlorosilane (TMSCl, 400 $\mathrm{mg}, 3.68 \mathrm{mmol}$ ) and imidazole $(833 \mathrm{mg}, 12.25$ mmol) at $0{ }^{\circ} \mathrm{C}$. After $2 \mathrm{~h}$, the mixture was quenched with water and then extracted with ether. The organic phase was washed with water, brine, and then dried over $\mathrm{Na}_{2} \mathrm{SO}_{4}$ and concentrated. The residue was chromatographed to give the desired substrate 1e (548 mg, 79\% yield): ${ }^{1} \mathrm{H}$ NMR: (300 $\left.\mathrm{MHz}, \mathrm{CDCl}_{3}\right) \delta 0.09(\mathrm{~s}, 9 \mathrm{H}), 1.51 \sim 1.84(\mathrm{~m}, 11 \mathrm{H}), 1.86 \sim 2.02(\mathrm{~m}, 1 \mathrm{H}), 2.62(\mathrm{~d}, 1 \mathrm{H}, \mathrm{J}=4.2 \mathrm{~Hz}), 2.68(\mathrm{~d}$, 
A Tandem Semipinacol/Schmidt Reaction Leading to Versatile and Efficient Approach to Azaquaternary Alkaloid Skeletons $\quad$ Peiming Gu, Yu-Ming Zhao, Yong Qiang Tu, " Yufei Ma, and Fumin Zhang

$1 \mathrm{H}, \mathrm{J}=4.2 \mathrm{~Hz}), 3.22 \sim 3.30(\mathrm{~m}, 2 \mathrm{H}) ;{ }^{13} \mathrm{C}$ NMR $\left(75 \mathrm{MHz}, \mathrm{CDCl}_{3}\right) \delta$ 2.1, 23.3, 23.4, 23.8, 26.8, 34.9, 36.9, 49.4, 51.6, 60.8, 87.1; HMRS (ESI, $\mathrm{M}^{+}+\mathrm{Na}, \mathrm{C}_{13} \mathrm{H}_{25} \mathrm{~N}_{3} \mathrm{O}_{2} \mathrm{NaSi}$ ) Calcd. : 306.1608; Found: 306.1611; Error: 1.0 ppm; IR $2096.3 \mathrm{~cm}^{-1}$.

A Typical Procedure for the Synthesis Substrate $1 \mathrm{f}$ was listed as another model (Methods B).<smiles>COc1cccc(C(C)(O[Na])C2(CCCN)CO2)c1</smiles>

Methanesulfonyl chloride $(0.128 \mathrm{~mL}, 1.65 \mathrm{mmol})$ was added to a solution of compound $5 \mathrm{c}$<smiles>C=C(CCCO)C(C)(O)c1cccc(OC)c1</smiles>

(prepared as for $5 \mathrm{e}, 1.347 \mathrm{~g}, 5.7 \mathrm{mmoL})$ and triethylamine $(1.2 \mathrm{~mL}$, $8.6 \mathrm{mmol})$ in $\mathrm{CH}_{2} \mathrm{Cl}_{2}(50 \mathrm{~mL})$ at $0{ }^{\circ} \mathrm{C}$. After $5 \mathrm{~min}$, the mixture was diluted with $\mathrm{CH}_{2} \mathrm{Cl}_{2}$ and washed with brine, and then dried $\left(\mathrm{Na}_{2} \mathrm{SO}_{4}\right)$ and concertrated to give the crude mesylate, which was diluted in dry pyridine $(20 \mathrm{~mL})$ and treated with hexamethyl disilazane (HMDS, $2.4 \mathrm{~mL}, 11.4$ mmol) and TMSCl $(0.98 \mathrm{~mL}, 7.7 \mathrm{mmoL})$ subsequently. The reaction mixture was stirred for 30 min and $\mathrm{H}_{2} \mathrm{O}(10 \mathrm{~mL})$ was added carefully. After dilution with ether $(150 \mathrm{~mL})$, the organic layer was separated, and washed with saturated $\mathrm{CuSO}_{4}$ solution, water, brine, dried over $\mathrm{Na}_{2} \mathrm{SO}_{4}$ and concentrated in vacuum, chromatographed gave a clear oil $(1.122 \mathrm{~g}, 51 \%)$.

The oil (1.122g $2.9 \mathrm{mmol})$ was dissolved in DMF $(5 \mathrm{~mL})$ and treated with sodium azide (470 $\mathrm{mg}$, $2.7 \mathrm{mmol})$ at room temperature. After $48 \mathrm{~h}$, the mixture was diluted with water $(10 \mathrm{~mL})$ and then extracted with ether $(150 \mathrm{~mL})$. The organic layer was washed with water, brine, and then dried over $\mathrm{Na}_{2} \mathrm{SO}_{4}$, concentrated. The residue (688 mg) was diluted into $\mathrm{CH}_{2} \mathrm{Cl}_{2}, \mathrm{NaHCO}_{3}(344 \mathrm{mg}, 4.1$ mmol) was added and then treated with $\mathrm{m}$-CPBA $(430 \mathrm{mg}, 2.5 \mathrm{mmol})$. After $2.5 \mathrm{~h}$, the reaction mixture was diluted with $\mathrm{CH}_{2} \mathrm{Cl}_{2}(100 \mathrm{~mL})$ and washed with saturated $\mathrm{K}_{2} \mathrm{CO}_{3}$ solution, dried over $\mathrm{Na}_{2} \mathrm{SO}_{4}$, concentrated and chromatographed to give the desired Siloxy-Epoxy-Azide 1c (649 mg, $64 \%$ yield). ${ }^{1} \mathrm{H}$ NMR: (300 MHz, $\left.\mathrm{CDCl}_{3}\right) \delta 0.08 \sim 0.19(9 \mathrm{H}), 1.30 \sim 1.71(7 \mathrm{H}), 2.59 \sim 2.67(1 \mathrm{H})$, 2.89 3.13(3H), 3.81(s, 3H), 6.78 7.27(3H); ${ }^{13} \mathrm{C}$ NMR (75 MHz, $\mathrm{CDCl}_{3}$ ) major $\delta$ 2.3, 23.9, 26.7, 47.7, 51.3, 55.1, 63.1, 77.8, 111.8, 112.2, 118.4, 128.8, 147.6, 159.2; HMRS (ESI, $\mathrm{M}^{+}+\mathrm{Na}$, $\mathrm{C}_{17} \mathrm{H}_{27} \mathrm{~N}_{3} \mathrm{O}_{3} \mathrm{NaSi}$ ) Calcd. : 372.1714 , Found: 372.1705 ; Error: $1.2 \mathrm{ppm}$; IR $2096.3 \mathrm{~cm}^{-1}$.<smiles>CC(O[Na])(c1ccccc1)C1(CCCN)CO1</smiles>

1a was prepared as for 1e, as a mixture of diastereomers: ${ }^{1} \mathrm{H}$ NMR $\left(300 \mathrm{MHz}, \mathrm{CDCl}_{3}\right)$ diastereoisomer mixture $(1: 1) \delta 0.08 \sim 0.17(9 \mathrm{H}), 1.27 \sim 1.72(7 \mathrm{H}), 2.60 \sim 2.68(1 \mathrm{H}), 2.89 \sim 3.14(3 \mathrm{H})$, 7.25 4.47(5H); ${ }^{13} \mathrm{C}$ NMR (75 MHz, $\mathrm{CDCl}_{3}$ ) diastereoisomer mixture (1:1) $\delta: 2.2,2.3,23.7,23.86$, 23.92, 25.0, 26.0, 26.7, 47.8, 48.9, 51.3, 51.4, 63.2, 63.3, 77.8, 78.9, 125.6, 126.0, 127.0, 127.1, 127.87, 127.95, 145.7, 146.0; HMRS (ESI, $\mathrm{M}^{+}+\mathrm{Na}, \mathrm{C}_{16} \mathrm{H}_{25} \mathrm{~N}_{3} \mathrm{O}_{2} \mathrm{NaSi}$ ) Calcd. : 342.1608 , Found: 342.1603, Error: 1.5 ppm; IR: $2095.5 \mathrm{~cm}^{-1}$. 
<smiles>COC(c1ccccc1)C1(CCCN)CO1</smiles>

1b was prepared as for 1c, as a mixture of diastereomers: ${ }^{1} \mathrm{H}$ NMR $\left(300 \mathrm{MHz}, \mathrm{CDCl}_{3}\right)$ diastereoisomer mixture $(1: 3) \delta 0.07 \sim 0.10(9 \mathrm{H}), 1.43 \sim 1.73(4 \mathrm{H}), 2.67 \sim 2.70(1 \mathrm{H}), 2.80 \sim 2.85(1 \mathrm{H})$, 3.13 3.19(2H), 4.49 4.51(1H), 7.26 7.39(5H); ${ }^{13} \mathrm{C} \mathrm{NMR} \mathrm{(75} \mathrm{MHz}, \mathrm{CDCl}_{3}$ ), diastereoisomer mixture, major $\delta$ 0.04, 23.7, 26.7, 50.1, 51.3, 61.0, 76.6, 126.7, 127.7, 128.1, 140.9; HMRS (ESI, $\mathrm{M}^{+}+\mathrm{Na}, \mathrm{C}_{15} \mathrm{H}_{23} \mathrm{~N}_{3} \mathrm{O}_{2} \mathrm{NaSi}$ ) Calcd. : 328.1452, Found: 328.1458, Error: $1.8 \mathrm{ppm}$; IR $2095.8 \mathrm{~cm}^{-1}$.<smiles>COC1(C2(CCCN)CO2)CCC1</smiles>

$1 \mathrm{~d}$

1d was prepared as for 1e: ${ }^{1} \mathrm{H}$ NMR $\left(300 \mathrm{MHz}, \mathrm{CDCl}_{3}\right) \delta 0.14(\mathrm{~s}, 9 \mathrm{H}), 1.48 \sim 1.58(\mathrm{~m}, 3 \mathrm{H})$, $1.74 \sim 1.80(\mathrm{~m}, 2 \mathrm{H}), 1.87(\mathrm{t}, 1 \mathrm{H}, \mathrm{J}=6.0 \mathrm{~Hz}), 1.98 \sim 2.08(\mathrm{~m}, 3 \mathrm{H}), 2.18(\mathrm{bs}, 1 \mathrm{H}), 2.66(\mathrm{~d}, 1 \mathrm{H}, \mathrm{J}=4.8 \mathrm{~Hz})$, $2.72(\mathrm{~d}, 1 \mathrm{H}, \mathrm{J}=4.8 \mathrm{~Hz}), 3.22 \sim 3.28(\mathrm{~m}, 2 \mathrm{H}) ;{ }^{13} \mathrm{C} \mathrm{NMR}\left(75 \mathrm{MHz}, \mathrm{CDCl}_{3}\right) \delta 1.9,13.2,23.9,25.5,31.9$, 33.1, 48.2, 51.6, 60.1, 79.4; HMRS (ESI, $\mathrm{M}^{+}+\mathrm{Na}, \mathrm{C}_{12} \mathrm{H}_{23} \mathrm{~N}_{3} \mathrm{O}_{2} \mathrm{NaSi}$ ), Calcd. : 292.1452, Found: 292.1448, Error: 1.4 ppm; IR $2096.3 \mathrm{~cm}^{-1}$.<smiles>COC1(C2(CCCN)CO2)CCCCC1</smiles>

1f was prepared as for 1e: ${ }^{1} \mathrm{H}$ NMR $\left(300 \mathrm{MHz}, \mathrm{CDCl}_{3}\right) \delta 0.13(\mathrm{~s}, 9 \mathrm{H}), 1.09 \sim 1.15(\mathrm{~m}, 1 \mathrm{H})$, $1.22 \sim 1.37(\mathrm{~m}, 2 \mathrm{H}), 1.42 \sim 1.65(\mathrm{~m}, 9 \mathrm{H}), 1.86 \sim 1.94(\mathrm{~m}, 2 \mathrm{H}), 2.55(\mathrm{~d}, 1 \mathrm{H}, \mathrm{J}=4.5 \mathrm{~Hz}), 2.80(\mathrm{~d}, 1 \mathrm{H}$, $\mathrm{J}=4.5 \mathrm{~Hz}), 3.21 \sim 3.33(\mathrm{~m}, 2 \mathrm{H}) ;{ }^{13} \mathrm{C} \mathrm{NMR}\left(75 \mathrm{MHz}, \mathrm{CDCl}_{3}\right) \delta$ 2.3, 21.48, 21.53, 23.6, 25.6, 25.7, 33.0, 33.6, 47.6, 51.7, 63.0, 76.3; HMRS (ESI, $\mathrm{M}^{+}+\mathrm{Na}, \mathrm{C}_{14} \mathrm{H}_{27} \mathrm{~N}_{3} \mathrm{O}_{2} \mathrm{NaSi}$ ) Calcd. : 320.1765, Found: 320.1764, Error: $0.3 \mathrm{ppm}$; IR $2096.6 \mathrm{~cm}^{-1}$.

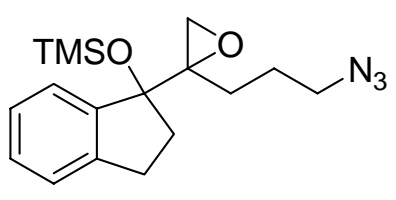

$1 \mathrm{~g}$ was prepared as for 1c, as a mixture of diastereomers: ${ }^{1} \mathrm{H}$ NMR $\left(300 \mathrm{MHz}, \mathrm{CDCl}_{3}\right)$ diastereoisomer mixture $\delta \quad-0.05(9 \mathrm{H}), \quad 1.48 \sim 2.23(6 \mathrm{H}), \quad 2.26 \sim 2.54(2 \mathrm{H}), \quad 2.79 \sim 3.35(4 \mathrm{H})$, 7.15 7.43(4H); ${ }^{13} \mathrm{C}$ NMR $\left(75 \mathrm{MHz}, \mathrm{CDCl}_{3}\right.$ ) diastereoisomer mixture (2:1) $\delta 1.6,23.9,26.4,29.8$, 38.3, 48.1, 51.6, 62.7, 87.3, 124.5, 126.0, 126.2, 128.6, 143.2, 143.8; HMRS (ESI, $\mathrm{M}^{+}+\mathrm{Na}$, $\mathrm{C}_{17} \mathrm{H}_{25} \mathrm{~N}_{3} \mathrm{O}_{2} \mathrm{NaSi}$ ) Calcd. : 354.1608; Found: 354.1595, Error: 3.7 ppm; IR $2095.8 \mathrm{~cm}^{-1}$.

\section{Procedure for the Synthesis Substrates $1 \mathrm{~h}$ and Spectral Data for $1 \mathrm{~h}$}

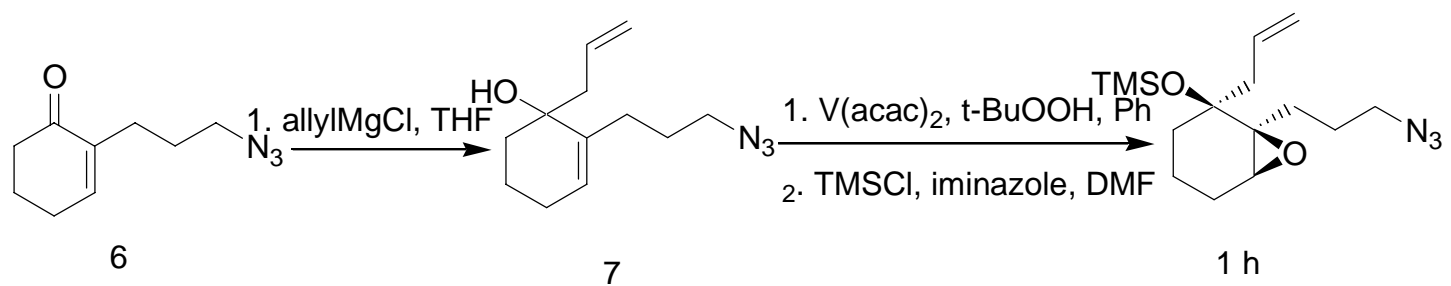


<smiles>C=CCC1(O)CCCC=C1CCCN</smiles>

Allylmagnesium chloride solution $(3.5 \mathrm{~mL}, 2 \mathrm{M}$ in THF, $7 \mathrm{mmol}$ ) was added to the known compoud $6^{2}(1.25 \mathrm{~g}, 7 \mathrm{mmol})$ in dry THF at $0{ }^{\circ} \mathrm{C}$. After $30 \mathrm{~min}$, the reaction mixture was diluted with water, and then extracted with ether. The organic phase was washed with $\mathrm{NH}_{4} \mathrm{Cl}$, water, brine, and then dried over $\mathrm{Na}_{2} \mathrm{SO}_{4}$ and concetrated. The residue was chromatographed to give the desired allyl alcohol 7 (773 mg, 50\% yield): ${ }^{1} \mathrm{H}$ NMR (300 MHz, $\left.\mathrm{CDCl}_{3}\right) \delta 1.60 \sim 1.64(\mathrm{~m}, 4 \mathrm{H})$, $1.73 \sim 1.82(\mathrm{~m}, 3 \mathrm{H}), 1.92 \sim 2.11(\mathrm{~m}, 3 \mathrm{H}), 2.21 \sim 2.32(\mathrm{~m}, 1 \mathrm{H}), 2.38(\mathrm{~d}, 2 \mathrm{H}, \mathrm{J}=6.9 \mathrm{~Hz}), 3.31(\mathrm{t}, 2 \mathrm{H}$, $\mathrm{J}=6.3 \mathrm{~Hz}), 5.10 \sim 5.15(\mathrm{~m}, 2 \mathrm{H}), 5.55(\mathrm{~s}, 1 \mathrm{H}), 5.69 \sim 5.84(\mathrm{~m}, 1 \mathrm{H}) ;{ }^{13} \mathrm{C} \mathrm{NMR}\left(75 \mathrm{MHz}, \mathrm{CDCl}_{3}\right) \delta 18.9$, 25.5, 27.4, 28.0, 36.1, 43.6, 51.4, 71.9, 118.6, 125.6, 133.7, 139.8 .<smiles>C=CC[C@]1(O)CCC[C@@H]2O[C@]21CCCN</smiles>

The allyl alcohol $7(773 \mathrm{mg}, 3.5 \mathrm{mmol})$, was diluted with benzene $(30 \mathrm{~mL})$, Vanadyl(IV) acetylacetonate $(50 \mathrm{mg}, 0.19 \mathrm{mmol})$ was added, following tert-butyl hydroperoxide $(t-\mathrm{BuOOH}$, $1.75 \mathrm{~mL}, 4.0 \mathrm{M}$ in pentane, $7.0 \mathrm{mmol}$ ), the reaction mixture was diluted with water, then extracted with ether. The organic phase was washed with saturated $\mathrm{NaHSO}_{3}$ solution, water, brine, and then dried over $\mathrm{Na}_{2} \mathrm{SO}_{4}$ and concentrated. The residue was chromatographed to give the $\mathrm{a}-$ hydroxy epoxide 8h (904 mg, 87\% yield): ${ }^{1} \mathrm{H}$ NMR $\left(300 \mathrm{MHz}, \mathrm{CDCl}_{3}\right) \delta 1.23 \sim 1.45(\mathrm{~m}, 5 \mathrm{H}), 1.47 \sim 1.98(\mathrm{~m}$, $4 \mathrm{H}), 2.06 \sim 2.16(\mathrm{~m}, 1 \mathrm{H}), 2.21(\mathrm{~s}, 1 \mathrm{H}), 2.28 \sim 2.35(\mathrm{~m}, 1 \mathrm{H}), 2.49 \sim 2.56(\mathrm{~m}, 1 \mathrm{H}), 3.21 \sim 3.34(\mathrm{~m}, 3 \mathrm{H})$, 5.01 5.15(m, 2H), 5.83 5.97(m, 1H); ${ }^{13} \mathrm{C}$ NMR $\left(75 \mathrm{MHz}, \mathrm{CDCl}_{3}\right) \delta 17.0,22.9,23.5,26.9$, 32.6, 40.4, 51.6, 60.9, 64.3, 73.2, 118.1, 133.2.<smiles>C=CC[C@]1(OS(C)(=O)=O)CCC[C@@H]2O[C@]21CCCN</smiles>

The a - Hydroxy epoxide $\mathbf{8 h}(626 \mathrm{mg}, 2.64 \mathrm{mmol})$ was treated with TMSCl $(0.578 \mathrm{~mL}, 5.28$ $\mathrm{mmol}$ ) and imidazole ( $854 \mathrm{mg}, 13.2 \mathrm{mmol}$ ) in $15 \mathrm{~mL}$ of DMF at room temperature. After $4 \mathrm{~h}$, the mixture was diluted with ether, and washed with water twice, and dried over $\mathrm{Na}_{2} \mathrm{SO}_{4}$, concentrated, and chromatographed to give the desired Siloxy-Epoxy-Azide $\mathbf{1 h}$ (750 mg, 92\% yield): ${ }^{1} \mathrm{H}$ NMR $\left(300 \mathrm{MHz}, \mathrm{CDCl}_{3}\right) \delta 0.12(\mathrm{~s}, 9 \mathrm{H}), 1.21 \sim 1.53(\mathrm{~m}, 4 \mathrm{H}), 1.56 \sim 1.90(\mathrm{~m}, 5 \mathrm{H}), 2.00 \sim 2.11(\mathrm{~m}, 1 \mathrm{H})$, $2.21 \sim 2.29(\mathrm{~m}, 1 \mathrm{H}), 2.41 \sim 2.47(\mathrm{dd}, 1 \mathrm{H}, \mathrm{J}=13.5 \mathrm{~Hz}, 3.3 \mathrm{~Hz}), 3.02(\mathrm{~s}, 1 \mathrm{H}), 3.18 \sim 3.33(\mathrm{~m}, 2 \mathrm{H})$, 5.01 5.09(m, 2H), 5.77 5.90(m, 1H); ${ }^{13} \mathrm{C}$ NMR $\left(75 \mathrm{MHz}, \mathrm{CDCl}_{3}\right) \delta$ 2.5, 18.3, 21.7, 23.3, 25.7, 29.9, 41.3, 51.7, 58.9, 64.1, 78.0, 117.1, 133.8; HRMS (ESI, $\mathrm{M}^{+}+\mathrm{Na}, \mathrm{C}_{15} \mathrm{H}_{27} \mathrm{~N}_{3} \mathrm{O}_{2} \mathrm{NaSi}$ ) Calcd. : 332.1765, Found: 332.1771, Error: 1.8 ppm; IR $2096.1 \mathrm{~cm}^{-1}$. 
A Tandem Semipinacol/Schmidt Reaction Leading to Versatile and Efficient Approach to Azaquaternary Alkaloid Skeletons $\quad$ Peiming Gu, Yu-Ming Zhao, Yong Qiang Tu, ${ }^{*}$ Yufei Ma, and Fumin Zhang

\section{Procedure for the Synthesis Substrates $1 \mathrm{i}$ and $1 \mathrm{j}$ and Spectral Data}

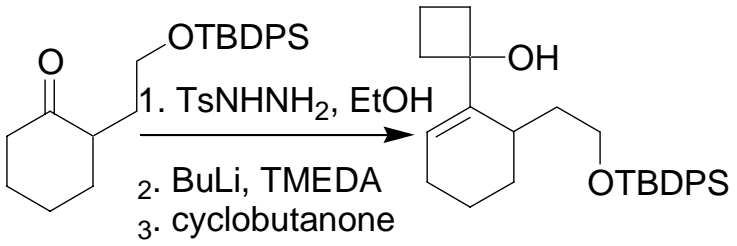

8<smiles></smiles>

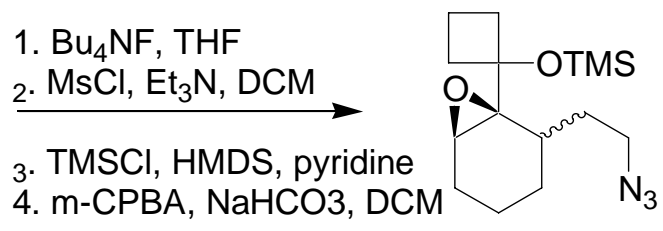

$1 \mathrm{i}$

The substituted cyclohexanone 8 ( $7.4 \mathrm{~g} 19.4 \mathrm{mmol})$ was added to a stirred solution of $\mathrm{TsNHNH}_{2}$ ( $3.8 \mathrm{~g}, 20.4 \mathrm{mmol}$ ) in $50 \mathrm{~mL}$ of ethanol. The solution was stirred for $5 \mathrm{~h}$ at room temperature, and $5 \mathrm{~mL}$ of water was added and the mixture was cooled for $12 \mathrm{~h}$ at $-20{ }^{\circ} \mathrm{C}$. the white solid was collected by filtration, washed with cold ethanol and then dried in vacuo ( $8.5 \mathrm{~g}, 80 \%$ yield). $n$-Butyllithium $(12.5 \mathrm{~mL}, 1.4 \mathrm{M}$ in hexane, $17.5 \mathrm{mmol})$ was slowly added to a stirred solution of tosylhydrazone $(2.7 \mathrm{~g}, 5 \mathrm{mmol})$ in $35 \mathrm{~mL}$ of TMEDA cooled at $-60{ }^{\circ} \mathrm{C}$ for $15 \mathrm{~min}$. The reaction mixture was warmed up to $10{ }^{\circ} \mathrm{C}$ and stirred for $10 \mathrm{~min}$, then cooled to $-40{ }^{\circ} \mathrm{C}$. cyclobutanone $(0.96 \mathrm{~mL}, 12.5 \mathrm{mmol})$ was added. The resulting mixture was warmed to room temperature for additional $30 \mathrm{~min}$ and poured into a mixture of water $(10 \mathrm{~mL})$-ether $(100 \mathrm{~mL})$. The organic layer was separated and washed with brine dried over $\mathrm{Na}_{2} \mathrm{SO}_{4}$, filtered and finally evaporated under reduced pressure, flash chromatographed to give allylic alcohol 9 (1.28 g, 57\% yield): ${ }^{1} \mathrm{H}$ NMR (300 MHz, d-acetone) $\delta 1.05(\mathrm{~s}, 9 \mathrm{H}), 1.41 \sim 1.62(\mathrm{~m}, 6 \mathrm{H}), 1.87 \sim 2.16(\mathrm{~m}, 5 \mathrm{H}), 2.17 \sim 2.40(\mathrm{~m}, 3 \mathrm{H})$, $2.59(\mathrm{bd}, 1 \mathrm{H}, \mathrm{J}=8.7 \mathrm{~Hz}), 3.74 \sim 3.80(\mathrm{~m}, 3 \mathrm{H}), 5.71(\mathrm{~d}, 1 \mathrm{H}, \mathrm{J}=3.6 \mathrm{~Hz}), 7.40 \sim 7.74(\mathrm{~m}, 10 \mathrm{H}) ;{ }^{13} \mathrm{C} \mathrm{NMR}$ (75 MHz, d-acetone) $\delta 13.8,18.2,19.1,25.2,26.6,26.9,30.2,35.3,35.9,37.4,62.8,78.2,120.8$, 128.0, 129.9, 134.1, 135.7, 145.6.<smiles>OCCC1CCCC=C1C1(O)CCC1</smiles>

The siloxyl ether 9 (1.2 g, $2.62 \mathrm{mmol})$ was treated with tetrabutylammonium fluoride trihydrate $(1.8 \mathrm{~g}, 5.71 \mathrm{mmol})$ at room temperature. After $3 \mathrm{~h}$, ether $(300 \mathrm{~mL})$ was added, and the mixture was washed with water and brine and then dried $\left(\mathrm{Na}_{2} \mathrm{SO}_{4}\right)$ and concentrated. Chromatography gave $514 \mathrm{mg}$ of the desired compound 10 (100\% yield): ${ }^{1} \mathrm{H}$ NMR (300 MHz, d-acetone) $\delta 1.40 \sim 1.68(\mathrm{~m}$, $6 \mathrm{H}), \quad 1.84 \sim 2.36(\mathrm{~m}, 6 \mathrm{H}), 2.50 \sim 2.52(\mathrm{~m}, 2 \mathrm{H}), 2.94(\mathrm{bs}, 1 \mathrm{H}), 3.54 \sim 3.64(\mathrm{~m}, 3 \mathrm{H}), 4.01(\mathrm{~s}, 1 \mathrm{H})$, 5.67 5.69(m, 1H); ${ }^{13} \mathrm{C}$ NMR (75 MHz, d-acetone) $\delta 13.7,18.2,25.1,27.5,30.4,35.3,36.5,37.4$, 60.7, 78.2, 120.4, 145.7; MS (EI) m/e 151, 135, 125, 109, 77.

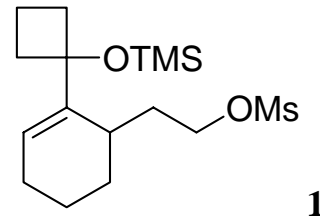


A Tandem Semipinacol/Schmidt Reaction Leading to Versatile and Efficient Approach to Azaquaternary Alkaloid Skeletons Peiming Gu, Yu-Ming Zhao, Yong Qiang Tu, ${ }^{*}$ Yufei Ma, and Fumin Zhang

Methanesulfonyl chloride $(0.198 \mathrm{~mL}, 2.55 \mathrm{mmol})$ was added to a solution of the compound $\mathbf{1 0}$ (500 mg, $2.55 \mathrm{mmol})$ and triethylamine $(0.355 \mathrm{~mL}, 2.55 \mathrm{mmol})$ in $\mathrm{CH}_{2} \mathrm{Cl}_{2}$ at $0{ }^{\circ} \mathrm{C}$. After $10 \mathrm{~min}$, the mixture was diluted with ether and washed with brine, and then dried $\left(\mathrm{Na}_{2} \mathrm{SO}_{4}\right)$ and concertrated to give the crude mesylate, which was diluted in dry pyridine $(15 \mathrm{~mL})$ and treated with hexamethyl disilazane (HMDS, $1.2 \mathrm{~mL}, 5.7 \mathrm{mmol})$ and TMSCl $(0.50 \mathrm{~mL}, 3.9 \mathrm{mmoL})$ subsequently. The reaction mixture was stirred for $30 \mathrm{~min}$ and $\mathrm{H}_{2} \mathrm{O}(10 \mathrm{~mL})$ was added carefully. After dilution with ether $(150 \mathrm{~mL})$, the organic layer was separated, and washed with saturated $\mathrm{CuSO}_{4}$ solution, water, brine, dried over $\mathrm{Na}_{2} \mathrm{SO}_{4}$ and concentrated in vacuum, gave a clear oil $\mathbf{1 1}$ (560 mg, 64\% yield): ${ }^{1} \mathrm{H}$ NMR (300 MHz, $\left.\mathrm{CDCl}_{3}\right) \delta 0.08(\mathrm{~s}, 9 \mathrm{H}), 1.43 \sim 1.61(\mathrm{~m}, 6 \mathrm{H}), 1.75 \sim 1.81(\mathrm{~m}$, $1 \mathrm{H}), 2.04 \sim 2.31(\mathrm{~m}, 7 \mathrm{H}), 2.43(\mathrm{bd}, 1 \mathrm{H}, \mathrm{J}=7.5 \mathrm{~Hz}), 2.98(\mathrm{~s}, 3 \mathrm{H}), 4.21 \sim 4.26(\mathrm{~m}, 2 \mathrm{H}), 5.71 \sim 5.74(\mathrm{~m}, 1 \mathrm{H})$; ${ }^{13} \mathrm{C}$ NMR $\left(75 \mathrm{MHz}, \mathrm{CDCl}_{3}\right) \delta 1.9,13.7,18.3,25.0,27.1,30.4,32.2,35.5,37.3,37.7,68.9,80.0$, 121.7, 142.6; MS (EI) m/e 256, 219, 145, 131, 117, 91, 79.<smiles>CS(=O)(=O)OC1(C2=CCCCC2CCN)CCC1</smiles>

12

The oil 11 (560 mg, $1.62 \mathrm{mmol}$ ) was dissolved in DMF and treated with sodium azide (263 $\mathrm{mg}$, $4.05 \mathrm{mmol}$ ) at room temperature. After $48 \mathrm{~h}$, the mixture was diluted with water and then extracted with ether. The organic phase was washed with water, brine, and then dried over $\mathrm{Na}_{2} \mathrm{SO}_{4}$ and concentrated. The residue was chromatographed to give the siloxy azide 12 (342 $\mathrm{mg}, 72 \%$ yield): ${ }^{1} \mathrm{H}$ NMR $\left(300 \mathrm{MHz}, \mathrm{CDCl}_{3}\right) \delta 0.11(\mathrm{~s}, 9 \mathrm{H}), 1.44 \sim 1.57(\mathrm{~m}, 6 \mathrm{H}), 1.61 \sim 1.85(\mathrm{~m}, 1 \mathrm{H}), 2.07 \sim 2.39(\mathrm{~m}$, $8 \mathrm{H}), 3.23 \sim 3.35(\mathrm{~m}, 2 \mathrm{H}), 5.73(\mathrm{~d}, 1 \mathrm{H}, \mathrm{J}=3.6 \mathrm{~Hz}),{ }^{13} \mathrm{C} \mathrm{NMR}\left(75 \mathrm{MHz}, \mathrm{CDCl}_{3}\right) \delta 1.9,13.8,18.4,25.1$, 27.0, 31.4, 32.0, 35.5, 37.8, 50.0, 80.1, 121.4, 143.2.<smiles>CS(=O)(=O)C1CC2OC1C21CCCCC1CCN</smiles>

The Siloxy-Azide $12(282 \mathrm{mg}, 0.96 \mathrm{mmol})$ was diluted into $\mathrm{CH}_{2} \mathrm{Cl}_{2}, \mathrm{NaHCO}_{3}(162 \mathrm{mg}, 1.93$ mmol) was added and then treated with m-CPBA $(85 \%, 197 \mathrm{mg}, 0.96 \mathrm{mmol})$. After 2.5h, the reaction mixture was diluted with $\mathrm{CH}_{2} \mathrm{Cl}_{2}$ and washed with saturated $\mathrm{K}_{2} \mathrm{CO}_{3}$ solution, dried over $\mathrm{Na}_{2} \mathrm{SO}_{4}$, concentrated and chromatographed to give the desired Sily-Epoxy-Azide 1i (271 mg, 91\% yield): ${ }^{1} \mathrm{H}$ NMR (300 MHz, $\mathrm{CDCl}_{3}$ ) diastereoisomer mixture (6:1) $\delta \quad 0.08 \sim 0.18(9 \mathrm{H})$, $1.13 \sim 1.58(5 \mathrm{H}), \quad 1.59 \sim 1.76(2 \mathrm{H}), 1.78 \sim 2.31(8 \mathrm{H}), 3.07 \sim 3.08(1 \mathrm{H}), 3.18 \sim 3.39(2 \mathrm{H}) ;{ }^{13} \mathrm{C}$ NMR $(75$ $\mathrm{MHz}, \mathrm{CDCl}_{3}$ ) diastereoisomer mixture, major $\delta$ 2.0, 13.8, 23.4, 25.0, 25.3, 28.6, 29.1, 31.8, 32.5, 49.8, 55.3, 63.2, 80.4; HRMS (ESI, $\mathrm{M}^{+}+\mathrm{Na}, \mathrm{C}_{15} \mathrm{H}_{27} \mathrm{~N}_{3} \mathrm{O}_{2} \mathrm{SiNa}$ ) Calcd. : 332.1765, Found: 332.1755, Error: 3.0 ppm; IR $2094.5 \mathrm{~cm}^{-1}$.

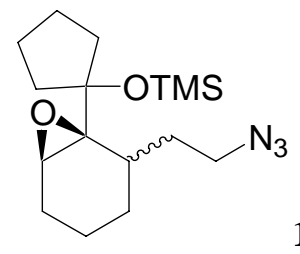

$1 \mathbf{j}$

1j was prepared as for $\mathbf{1 i}$, as a mixture of diastereomers: ${ }^{1} \mathrm{H}$ NMR $\left(300 \mathrm{MHz}, \mathrm{CDCl}_{3}\right)$ 
A Tandem Semipinacol/Schmidt Reaction Leading to Versatile and Efficient Approach to Azaquaternary Alkaloid Skeletons Peiming Gu, Yu-Ming Zhao, Yong Qiang Tu, ${ }^{*}$ Yufei Ma, and Fumin Zhang diastereoisomer mixture $(8: 1) \quad \delta \quad 0.09(9 \mathrm{H}), 1.11 \sim 1.21(1 \mathrm{H}), 1.24 \sim 1.38(2 \mathrm{H}), 1.42 \sim 1.48(1 \mathrm{H})$, $1.54 \sim 1.75(10 \mathrm{H}), \quad 1.83 \sim 1.93(1 \mathrm{H}), 2.05(\mathrm{bd}, 1 \mathrm{H}, \mathrm{J}=13.5 \mathrm{~Hz}), 2.39 \sim 2.44(1 \mathrm{H}), 2.93 \sim 3.03(1 \mathrm{H}$, $3.20 \sim 3.38(2 \mathrm{H}) ;{ }^{13} \mathrm{C}$ NMR $\left(75 \mathrm{MHz}, \mathrm{CDCl}_{3}\right) \delta 2.1,14.3,22.9,23.6,25.0,25.1,28.0,28.7,33.8$, 36.8, 49.9, 56.4, 63.8, 87.4; HRMS (ESI, $\mathrm{M}^{+}+\mathrm{Na}, \mathrm{C}_{16} \mathrm{H}_{29} \mathrm{~N}_{3} \mathrm{O}_{2} \mathrm{SiNa}$ ) Calcd. : 346.1921 , Found: 346.1922, Error: $0.3 \mathrm{ppm}$; IR $2094.4 \mathrm{~cm}^{-1}$.

\section{Procedure for the Tandem Semipinacol/intramolecular Aube's type Schmidt reaction}

To a cooled $\left(-78^{\circ} \mathrm{C}\right)$ solution of the Siloxy-Epoxy-Azide $1(0.3 \mathrm{~m} \mathrm{~mol})$ in $\mathrm{CH}_{2} \mathrm{Cl}_{2}(3 \mathrm{ml})$ under argon was added $\mathrm{TiCl}_{4}\left(0.66 \mathrm{ml}, 1 \mathrm{M}\right.$ in $\left.\mathrm{CH}_{2} \mathrm{Cl}_{2}\right)$. The result mixture was stirred $-78{ }^{\circ} \mathrm{C}$ for 15 minutes. Then the mixture was warmed slowly to room temperature $\left(25^{\circ} \mathrm{C}\right)$ for additional times, then it was quenched with $1 \mathrm{ml}$ water. The mixture was partitioned between water and $50 \mathrm{ml}$ $\mathrm{CH}_{2} \mathrm{Cl}_{2}$. The organic layer was collected, dried $\left(\mathrm{Na}_{2} \mathrm{SO}_{4}\right)$, filtered, and concentrated to give a solid 2. Chromatography afforded the desired products.

\section{Spectral Data for Tandem Products}<smiles>CC(=O)N1CCCC1(CO)c1ccccc1</smiles>

2a: Siloxy-Epoxy-Azide 1a run in the general procedure. The mixture was stirred at room temperature for $1.5 \mathrm{~h}$, and it afforded $2 \mathrm{a}$ as a white solid in $95 \%$ yield: ${ }^{1} \mathrm{H} \mathrm{NMR}\left(300 \mathrm{MHz}, \mathrm{CDCl}_{3}\right.$ ) $\delta 1.66 \sim 1.94(\mathrm{~m}, 2 \mathrm{H}), 1.96 \sim 2.01(\mathrm{~m}, 2 \mathrm{H}), 2.24(\mathrm{~s}, 3 \mathrm{H}), 3.60 \sim 3.78(\mathrm{~m}, 2 \mathrm{H}), 3.90(\mathrm{~d}, 1 \mathrm{H}, \mathrm{J}=12.3 \mathrm{~Hz})$, 4.26(d, $1 \mathrm{H}, \mathrm{J}=12.0 \mathrm{~Hz}), 7.13 \sim 7.37(\mathrm{~m}, 5 \mathrm{H}) ;{ }^{13} \mathrm{C} \mathrm{NMR}\left(75 \mathrm{MHz}, \mathrm{CDCl}_{3}\right) \delta 21.2,23.9,39.8,50.7$, 68.3, 74.2, 125.9, 126.7, 128.3, 140.4, 171.5; MS (EI) m/e 219, 201, 188, 146; HMRS (ESI, $\mathrm{M}^{+}+\mathrm{Na}, \mathrm{C}_{13} \mathrm{H}_{17} \mathrm{NO}_{2} \mathrm{Na}$ ) Calcd. : 242.1151, Found: 242.1148, Error: $1.2 \mathrm{ppm}$; IR $1626.0 \mathrm{~cm}^{-1}$.<smiles>O=CN1CCCC1(CO)c1ccccc1</smiles>

2b: Siloxy-Epoxy-Azide $\mathbf{1 b}$ run in the general procedure. The mixture was stirred at room temperature for $0.5 \mathrm{~h}$, and it afforded $2 \mathbf{b}$ as a white solid in $80 \%$ yield: ${ }^{1} \mathrm{H}$ NMR (300 MHz, $\left.\mathrm{CDCl}_{3}\right) \delta 1.65 \sim 1.75(\mathrm{~m}, 1 \mathrm{H}), \quad 1.83 \sim 1.92(\mathrm{~m}, 1 \mathrm{H}), \quad 2.00 \sim 2.10(\mathrm{~m}, 1 \mathrm{H}), 2.30 \sim 2.39(\mathrm{~m}, 1 \mathrm{H})$, $3.54 \sim 3.64(\mathrm{~m}, 1 \mathrm{H}), 3.69 \sim 3.78(\mathrm{~m}, 1 \mathrm{H}), 3.92(\mathrm{~d}, 1 \mathrm{H}, \mathrm{J}=12.3 \mathrm{~Hz}), 4.25(\mathrm{~d}, 1 \mathrm{H}, \mathrm{J}=12.3 \mathrm{~Hz})$, 7.18 7.38(m, 5H), 8.18(s, 1H); ${ }^{13} \mathrm{C}$ NMR $\left(75 \mathrm{MHz}, \mathrm{CDCl}_{3}\right) \delta 21.0,38.0,45.6,66.2,70.3,125.7$, 127.4, 128.7, 142.9, 163.0; MS (EI) m/e 174, 146, 104; HMRS (ESI, $\mathrm{M}^{+}+\mathrm{H}, \mathrm{C}_{12} \mathrm{H}_{16} \mathrm{NO}_{2}$ ) Calcd. : 206.1176, Found: 206.1176, Error: 0 ppm; IR $1647.3 \mathrm{~cm}^{-1}$. 
<smiles>COc1cccc(C2(CO)CCCN2C(C)=O)c1</smiles>

2c: Siloxy-Epoxy-Azide 1c run in the general procedure. The mixture was stirred at room temperature for $10 \mathrm{~h}$, and it afforded $2 \mathrm{c}$ as a white solid in $77 \%$ yield: ${ }^{1} \mathrm{H}$ NMR $\left(300 \mathrm{MHz}, \mathrm{CDCl}_{3}\right.$ ) $\delta 1.66 \sim 1.84(\mathrm{~m}, 2 \mathrm{H}), 1.88 \sim 2.05(\mathrm{~m}, 2 \mathrm{H}), 2.21(\mathrm{~s}, 3 \mathrm{H}), 3.57 \sim 3.76(\mathrm{~m}, 2 \mathrm{H}), 3.78(\mathrm{~s}, 3 \mathrm{H}), 3.87(\mathrm{~d}, 1 \mathrm{H}$, $\mathrm{J}=12.3 \mathrm{~Hz}), 4.20(\mathrm{t}, 1 \mathrm{H}, \mathrm{J}=10.5 \mathrm{~Hz}), 5.65(\mathrm{bd}, 1 \mathrm{H}), 6.66(\mathrm{t}, 1 \mathrm{H}), 6.74 \sim 6.78(\mathrm{~m}, 2 \mathrm{H}), 7.26(\mathrm{~m}, 2 \mathrm{H}),{ }^{13} \mathrm{C}$ $\operatorname{NMR}\left(75 \mathrm{MHz}, \mathrm{CDCl}_{3}\right) \delta 21.1,23.9,39.7,50.6,55.0,68.3,74.2,111.0,112.9,118.4,129.3,142.4$, 159.4, 171.3; MS (EI) m/e 249, 231, 218, 176; HMRS (ESI, $\mathrm{M}^{+}+\mathrm{Na}, \mathrm{C}_{14} \mathrm{H}_{19} \mathrm{NO}_{3} \mathrm{Na}$ ) Calcd. : 272.1257, Found: 272.1250, Error: 2.6 ppm; IR $1626.4 \mathrm{~cm}^{-1}$.<smiles>O=C1CCCC2(CO)CCCN12</smiles>

2d: Siloxy-Epoxy-Azide 1d run in the general procedure. The mixture was stirred at room temperature for $3 \mathrm{~h}$, and it afforded $\mathbf{2 d}$ as a white solid in $77 \%$ yield. ${ }^{1} \mathrm{H}$ NMR $\left(300 \mathrm{MHz}, \mathrm{CDCl}_{3}\right)$ $\delta 1.28 \sim 1.41(\mathrm{~m}, 1 \mathrm{H}), 1.47 \sim 1.58(\mathrm{~m}, 1 \mathrm{H}), 1.72 \sim 1.82(\mathrm{~m}, 1 \mathrm{H}), 1.86 \sim 2.00(\mathrm{~m}, 3 \mathrm{H}), 2.20 \sim 2.45(\mathrm{~m}, 4 \mathrm{H})$, $2.55(\mathrm{bs}, 1 \mathrm{H}), 3.34 \sim 3.44(\mathrm{~m}, 2 \mathrm{H}), 3.51(\mathrm{~d}, 1 \mathrm{H}, 11.1 \mathrm{~Hz}), 3.72 \sim 3.81(\mathrm{~m}, 1 \mathrm{H}) ;{ }^{13} \mathrm{C} \mathrm{NMR}(75 \mathrm{MHz}$, $\left.\mathrm{CDCl}_{3}\right) \delta 17.5,20.1,30.2,30.3,35.6,44.8,63.6,64.8,170.4$; MS (EI) m/e 169, 138, 110, 84, 55; HMRS (ESI, $\mathrm{M}^{+}+\mathrm{H}, \mathrm{C}_{9} \mathrm{H}_{16} \mathrm{NO}_{2}$ ) Calcd. : 170.1176, Found: 170.1173, Error: 1.8 ppm; IR 1607.8 $\mathrm{cm}^{-1}$.<smiles>O=C1CCCCC2(CO)CCCN12</smiles>

2e: Siloxy-Epoxy-Azide 1e run in the general procedure. The mixture was stirred at room temperature for $0.5 \mathrm{~h}$, and it afforded $2 \mathrm{e}$ as a white solid in $67 \%$ yield. ${ }^{1} \mathrm{H}$ NMR $\left(400 \mathrm{MHz}, \mathrm{CDCl}_{3}\right)$ $\delta 1.44 \sim 1.55(\mathrm{~m}, 2 \mathrm{H}), 1.63 \sim 1.79(\mathrm{~m}, 5 \mathrm{H}), 1.80 \sim 1.89(\mathrm{~m}, 1 \mathrm{H}), 2.01 \sim 2.06(\mathrm{dt}, \mathrm{J}=10.8 \mathrm{~Hz}, 2.4 \mathrm{~Hz}, 1 \mathrm{H})$, $2.25 \sim 2.30(\mathrm{~m}, 1 \mathrm{H}), 2.41 \sim 2.47(\mathrm{ddd}, \mathrm{J}=10.8 \mathrm{~Hz}, 3.9 \mathrm{~Hz}, 1.5 \mathrm{~Hz}, 1 \mathrm{H}), 2.63 \sim 2.70(\mathrm{td}, \mathrm{J}=10.8 \mathrm{~Hz}, 2.1 \mathrm{~Hz}$, $1 \mathrm{H}), 3.50 \sim 3.58(\mathrm{~m}, 2 \mathrm{H}), 3.63(\mathrm{~d}, 1 \mathrm{H}, \mathrm{J}=11.6 \mathrm{~Hz}), 3.82(\mathrm{~d}, 1 \mathrm{H}, \mathrm{J}=11.6 \mathrm{~Hz}), 4.50(\mathrm{bs}, 1 \mathrm{H}) ;{ }^{13} \mathrm{C}$ NMR $\left(100 \mathrm{MHz}, \mathrm{CDCl}_{3}\right) \delta 20.9,23.6(2 \mathrm{C}), 35.8,37.0,38.9,49.4,63.9,65.6,173.9 ; \mathrm{MS}$ (EI) m/e 183, 152; HMRS (ESI, $\mathrm{M}^{+}+\mathrm{Na}, \mathrm{C}_{10} \mathrm{H}_{17} \mathrm{NO}_{2} \mathrm{Na}$ ) Calcd. : 206.1151, Found: 206.1148, Error: 1.5 ppm; IR $1600.5 \mathrm{~cm}^{-1}$. 


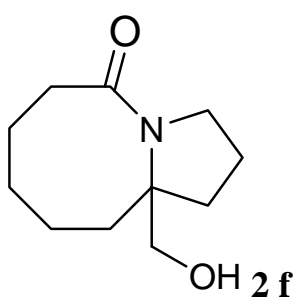

2f: Siloxy-Epoxy-Azide 1f run in the general procedure. The mixture was stirred at room temperature for $8 \mathrm{~h}$, and it afforded $\mathbf{2 f}$ as a white solid in $66 \%$ yield: ${ }^{1} \mathrm{H}$ NMR $\left(300 \mathrm{MHz}, \mathrm{CDCl}_{3}\right.$ ) $\delta 1.42 \sim 1.68(\mathrm{~m}, 4 \mathrm{H}), 1.72 \sim 1.96(\mathrm{~m}, 7 \mathrm{H}), 2.17 \sim 2.25(\mathrm{~m}, 2 \mathrm{H}), 2.92 \sim 3.02(\mathrm{~m}, 1 \mathrm{H}), 3.48 \sim 3.64(\mathrm{~m}, 4 \mathrm{H})$, 4.60(bs, $1 \mathrm{H}) ;{ }^{13} \mathrm{C}$ NMR $\left(75 \mathrm{MHz}, \mathrm{CDCl}_{3}\right) \delta 19.7,21.5,25.0,25.3,33.9,34.0,35.6,48.7,67.3$, 68.2, 174.2; MS (EI) m/e 197, 166; HMRS (ESI, $\mathrm{M}^{+}+\mathrm{H}, \mathrm{C}_{11} \mathrm{H}_{20} \mathrm{NO}_{2}$ ) Calcd. : 198.1489, Found: 198.1485, Error: $2.0 \mathrm{ppm}$; IR $1599.5 \mathrm{~cm}^{-1}$.<smiles>O=C1CCc2ccccc2C2(CO)CCCN12</smiles><smiles>N#CCCC1(CO)CCc2ccccc2C1=O</smiles>

2g: Siloxy-Epoxy-Azide $\mathbf{1 g}$ run in the general procedure. The mixture was stirred at room temperature for $3 \mathrm{~h}$, and it afforded $\mathbf{2 g}$ as a white solid in $61 \%$ yield and a byproduct $\mathbf{2 g}$ ' in $20 \%$ yield: $2 \mathrm{~g}{ }^{1} \mathrm{H}$ NMR $\left(300 \mathrm{MHz}, \mathrm{CDCl}_{3}\right) \delta 1.73 \sim 1.82(\mathrm{~m}, 1 \mathrm{H}), 2.11 \sim 2.24(\mathrm{~m}, 1 \mathrm{H}), 2.34 \sim 2.44(\mathrm{dt}$, $\mathrm{J}=15.0 \mathrm{~Hz}, 7.8 \mathrm{~Hz}, 1 \mathrm{H}), 2.53 \sim 2.63(\mathrm{~m}, 1 \mathrm{H}), 2.84 \sim 3.19(\mathrm{~m}, 4 \mathrm{H}), 3.48 \sim 3.58(\mathrm{dt}, \mathrm{J}=11.7 \mathrm{~Hz}, 8.7 \mathrm{~Hz}, 1 \mathrm{H})$, $3.76(\mathrm{~d}, 1 \mathrm{H}, \mathrm{J}=12.3 \mathrm{~Hz}), 3.88 \sim 3.96(\mathrm{td}, \mathrm{J}=9.3 \mathrm{~Hz}, 3.0 \mathrm{~Hz}, 1 \mathrm{H}), 4.11(\mathrm{~d}, 1 \mathrm{H}, \mathrm{J}=12.3 \mathrm{~Hz}), 4.96(\mathrm{bs}, 1 \mathrm{H})$, 7.08 7.26(m, 4H); ${ }^{13} \mathrm{C}$ NMR (75 MHz, $\left.\mathrm{CDCl}_{3}\right) \delta 20.6,30.8,35.3,41.4,49.5,70.5,71.4,126.3$, 126.9, 129.2, 130.9, 137.9, 139.4, 174.7; MS (EI) m/e 231, 200, 156, 111; HMRS (ESI, ${ }^{+}+\mathrm{Na}$, $\mathrm{C}_{14} \mathrm{H}_{17} \mathrm{NO}_{2} \mathrm{Na}$ ) Calcd. : 254.1151, Found: 254.1157, Error: $2.4 \mathrm{ppm}$; IR $1617.6 \mathrm{~cm}^{-1} .2 \mathrm{~g}{ }^{1} \mathrm{H}$ NMR $\left(300 \mathrm{MHz}, \mathrm{CDCl}_{3}\right) \delta 1.57 \sim 1.81(\mathrm{~m}, 4 \mathrm{H}), 1.89 \sim 1.97(\mathrm{~m}, 1 \mathrm{H}), 2.03 \sim 2.13(\mathrm{~m}, 1 \mathrm{H}), 2.90 \sim 2.98(\mathrm{~m}, 2 \mathrm{H})$, 3.04 3.39(m, 3H), 3.71(s, 2H), 7.17(d, 1H), 7.26(t, 1H), 7.47 7.53(m, 1H), 8.01(d, $1 \mathrm{H}) ;{ }^{13} \mathrm{C} \mathrm{NMR}$ $\left(75 \mathrm{MHz}, \mathrm{CDCl}_{3}\right) \delta 23.3,24.7,27.3,28.0,48.6,51.6,66.1,126.8,127.8,128.8,131.3,133.8$, 143.2, 203.7.<smiles>C=CC[C@]12CCCN1C(=O)CCC[C@H]2O</smiles>

2h: Siloxy-Epoxy-Azide $\mathbf{1 h}$ run in the general procedure. The mixture was stirred at room temperature for $1 \mathrm{~h}$, and it afforded $2 \mathbf{h}$ as a white solid in $92 \%$ yield: ${ }^{1} \mathrm{H} \mathrm{NMR}\left(300 \mathrm{MHz}, \mathrm{CDCl}_{3}\right)$ $\delta \quad 1.57 \sim 1.92(\mathrm{~m}, 5 \mathrm{H}), 1.97 \sim 2.06(\mathrm{~m}, 2 \mathrm{H}), 2.31 \sim 2.66(\mathrm{~m}, 6 \mathrm{H}), 3.54 \sim 3.59(\mathrm{~m}, 2 \mathrm{H}), 3.76(\mathrm{bs}, 1 \mathrm{H})$, 5.11 5.16(m, 2H), 5.62 5.76(m, 1H); ${ }^{13} \mathrm{C}$ NMR: $\left(75 \mathrm{MHz}, \mathrm{CDCl}_{3}\right), \delta: 16.6,21.5,30.7,36.3,37.3$, 40.6, 50.2, 67.7, 71.3, 119.3, 132.3, 173.5; MS (EI) m/e 209, 180, 168, 110; HRMS (ESI, $\mathrm{M}^{+}+\mathrm{Na}$, $\mathrm{C}_{12} \mathrm{H}_{19} \mathrm{NO}_{2} \mathrm{Na}$ ) Calcd. : 232.1308, Found: 232.1312, Error: 1.7 ppm; IR $1601.4 \mathrm{~cm}^{-1}$. 
<smiles>CCCN1C(=O)CCCC12CCCC2</smiles>

2i: Siloxy-Epoxy-Azide 1i (mixture $\alpha / \beta=1: 6$ ) run in the general procedure. The mixture was stirred at room temperature for $3 \mathrm{~h}$, and it afforded respectively $2 \mathbf{i}$ (mixture $\alpha / \beta=1: 7, \beta$ isomer was confirmed by $\mathrm{X}$ ray analysis) as white solids in $68 \%$ and $10 \%$ yields: $2 \mathrm{i} 1{ }^{1} \mathrm{H}$ NMR $(300 \mathrm{MHz}$, $\left.\mathrm{CDCl}_{3}\right) \delta 1.24 \sim 1.34(\mathrm{~m}, \quad 2 \mathrm{H}), \quad 1.40 \sim 1.76(\mathrm{~m}, \quad 5 \mathrm{H}), \quad 1.83 \sim 1.94(\mathrm{~m}, \quad 4 \mathrm{H}), \quad 1.98 \sim 2.07(\mathrm{~m}, \quad 1 \mathrm{H})$, $2.18 \sim 2.43(\mathrm{~m}, 3 \mathrm{H}), 3.52 \sim 3.57(\mathrm{~m}, 2 \mathrm{H}), 3.98(\mathrm{~s}, 1 \mathrm{H}), 4.66(\mathrm{bs}, 1 \mathrm{H}) ;{ }^{13} \mathrm{C} \mathrm{NMR}\left(75 \mathrm{MHz}, \mathrm{CDCl}_{3}\right) \delta$ 13.9, 17.8, 24.5, 25.7, 29.8, 29.9, 31.1, 45.9, 46.4, 64.4, 69.6, 170.4; MS (EI) m/e 209, 150, 137, 122, 84; HRMS (ESI, $\mathrm{M}^{+}+\mathrm{Na}, \mathrm{C}_{12} \mathrm{H}_{19} \mathrm{NO}_{2} \mathrm{Na}$ ) Calcd. : 232.1308, Found: 232.1311, Error: 1.3 ppm; IR $1621.4 \mathrm{~cm}^{-1} ; .2 \mathrm{i} 2 \mathrm{HNMR}\left(400 \mathrm{MHz}, \mathrm{CDCl}_{3}\right) \delta 1.18 \sim 1.31(\mathrm{~m}, 2 \mathrm{H}), 1.19 \sim 1.51(\mathrm{~m}, 2 \mathrm{H})$, $1.55 \sim 1.79(\mathrm{~m}, 6 \mathrm{H}), 1.70 \sim 1.91(\mathrm{~m}, 4 \mathrm{H}), 2.28 \sim 2.38(\mathrm{~m}, 1 \mathrm{H}), 2.43 \sim 2.52(\mathrm{~m}, 1 \mathrm{H}), 2.66 \sim 2.73(\mathrm{~m}, 1 \mathrm{H})$, 3.04 3.12(m, 1H), 3.97 4.02(m, 1H), 4.26(s, 1H); CNMR (100 MHz, $\left.\mathrm{CDCl}_{3}\right) \delta$ 15.0, 21.1, 24.67, $24.74,26.3,28.6,29.0,41.2,48.3,67.2,68.3,174.3$.

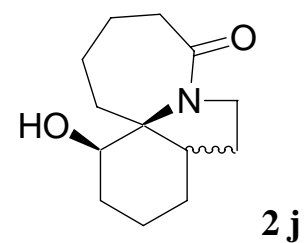

2j: Siloxy-Epoxy-Azide $\mathbf{1 j}$ (mixture $\alpha / \beta=1: 8$ ) run in the general procedure. The mixture was stirred at room temperature for $1 \mathrm{~h}$, and it afforded $2 \mathrm{j}$ with a minor diastereoisomer mixture $(\alpha / \beta=1: 8)$ as white solids in combined $93 \%$ yields. ${ }^{1} \mathrm{H}$ NMR $\left(300 \mathrm{MHz}, \mathrm{CDCl}_{3}\right) \delta 1.23 \sim 1.32(\mathrm{~m}$, $1 \mathrm{H}), 1.37 \sim 1.72(\mathrm{~m}, 9 \mathrm{H}), 1.77 \sim 2.02(\mathrm{~m}, 4 \mathrm{H}), 2.24 \sim 2.37(\mathrm{~m}, 2 \mathrm{H}), 2.55 \sim 2.65(\mathrm{~m}, 1 \mathrm{H}), 3.29 \sim 3.39(\mathrm{~m}$, $1 \mathrm{H}), 3.61 \sim 3.70(\mathrm{~m}, 1 \mathrm{H}), 4.81(\mathrm{~s}, 1 \mathrm{H}), 4.92(\mathrm{bs}, 1 \mathrm{H}) ;{ }^{13} \mathrm{C} \mathrm{NMR}\left(75 \mathrm{MHz}, \mathrm{CDCl}_{3}\right) \delta 13.8,22.2,23.4$, 24.5, 25.5, 34.5, 35.6, 45.7, 47.7, 65.7, 68.1, 173.4; MS (EI) m/e 223, 164, 151, 136, 122, 95; HRMS (ESI, $\mathrm{M}^{+}+\mathrm{Na}, \mathrm{C}_{13} \mathrm{H}_{21} \mathrm{NO}_{2} \mathrm{Na}$ ) Calcd. : 246.1465, Found: 246.1468, Error: 1.2 ppm; IR $1613.9 \mathrm{~cm}^{-1}$. 


\section{Synthesis of azaquaternary 5-7-5 ring skeleton from $2 \mathrm{~h}$}

$2 \mathrm{~h}$

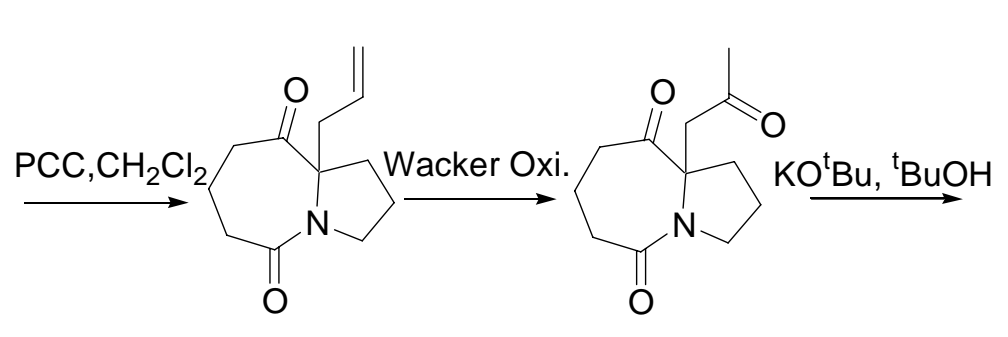

$3 \mathrm{~h}$
$4 \mathrm{~h}$

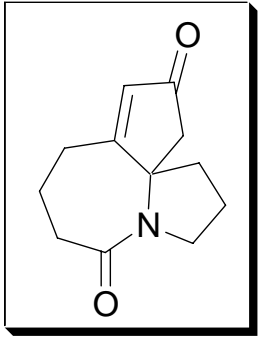

$5 \mathrm{~h}$

Procedure for Synthesis the Tricyclic Lactam $5 \mathbf{h}$ :<smiles>C=CCC12CCCN1C(=O)CCCC2=O</smiles>

PCC (250 mg, $1.16 \mathrm{mmol})$, silica (250 mg), and NaOAc (100 mg, $1.22 \mathrm{mmol})$ were well mixed, then added into the solution of $\mathbf{2 h}(132 \mathrm{mg}, 0.63 \mathrm{mmol})$ in $\mathrm{CH}_{2} \mathrm{Cl}_{2}$. After $18 \mathrm{~h}$, the mixture was subjected to a column of $\mathrm{Al}_{2} \mathrm{O}_{3}$, washed with $\mathrm{CHCl}_{3}$, the organic solvent was evaporated and chromatographed to give the ketone $8 \mathrm{c}\left(115 \mathrm{mg}, 88 \%\right.$ yield): ${ }^{1} \mathrm{H} \mathrm{NMR}\left(300 \mathrm{MHz}, \mathrm{CDCl}_{3}\right) \delta$ $1.87 \sim 2.29(\mathrm{~m}, 6 \mathrm{H}), 2.30 \sim 2.39(\mathrm{~m}, 2 \mathrm{H}), 2.44 \sim 2.57(\mathrm{~m}, 2 \mathrm{H}), 2.61 \sim 2.68(\mathrm{~m}, 1 \mathrm{H}), 2.79 \sim 2.89(\mathrm{~m}, 1 \mathrm{H})$, 3.57 3.64(m, 1H), 3.68 3.77(m, 1H), 5.07 5.13(m, 2H), 5.71 5.85(m, $1 \mathrm{H}) ;{ }^{13} \mathrm{C}$ NMR $(75 \mathrm{MHz}$, $\left.\mathrm{CDCl}_{3}\right) \delta 21.1,22.9,33.3,35.2,37.2,39.1,47.3,73.9,119.6,132.6,170.9,211.7$; MS (EI) m/e 207, 166, 138, 109, 96, 83, 70, 57, 41; HRMS (ESI, $\mathrm{M}^{+}+\mathrm{Na}, \mathrm{C}_{12} \mathrm{H}_{17} \mathrm{NO}_{2} \mathrm{Na}$ ) Calcd. : 230.1151, Found: 230.1157, Error: $2.6 \mathrm{ppm}$; IR 1645.8, $1714.0 \mathrm{~cm}^{-1}$.<smiles>CC(=O)CC12CCCN1C(=O)CCCC2=O</smiles>

$4 \mathrm{~h}$

$\mathrm{PdCl}_{2}(12 \mathrm{mg}, 0.07 \mathrm{mmol})$ and $\mathrm{Cu}_{2} \mathrm{Cl}_{2}(32 \mathrm{mg}, 0.16 \mathrm{mmol})$ were added into a mixture of DMF and water $(\mathrm{v} / \mathrm{v}=7: 1,2 \mathrm{~mL})$. Then saturated with oxygen for $2 \mathrm{~h}$. $3 \mathbf{h}(50 \mathrm{mg}, 0.24 \mathrm{mmol})$ was added to the mixture, and the mixture was heated to $60{ }^{\circ} \mathrm{C}$ for $3 \mathrm{~h}$. the solution was acidified to litmus with $10 \%$ hydrochloric acid $(5 \mathrm{~mL})$ and extracted 3 times with $20 \mathrm{~mL} \mathrm{CHCl} 3$, dried over $\mathrm{Na}_{2} \mathrm{SO}_{4}$, concentrated, and chromatographed to give the diketone $4 \mathbf{h}$ (45 mg, 84\%): ${ }^{1} \mathrm{H}$ NMR (300 MHz, $\left.\mathrm{CDCl}_{3}\right) \delta 1.88 \sim 2.20(\mathrm{~m}, 5 \mathrm{H}), 2.24(\mathrm{~s}, 2 \mathrm{H}), 2.29 \sim 2.40(\mathrm{~m}, 1 \mathrm{H}), 2.43 \sim 2.55(\mathrm{~m}, 3 \mathrm{H}), 2.64 \sim 2.69(\mathrm{~m}$, 1H), 2.82 2.95(m, 2H), 3.62 3.74(m, 2H); ${ }^{13} \mathrm{C} \mathrm{NMR}\left(75 \mathrm{MHz}, \mathrm{CDCl}_{3}\right) \delta 21.3,22.9,32.8,33.3$, 34.7, 35.2, 44.6, 47.1, 74.2, 170.4, 205.4, 210.8; MS (EI) m/e 223, 195, 180, 167, 152, 138, 126, 96; HRMS (ESI, $\mathrm{M}^{+}+\mathrm{Na}, \mathrm{C}_{12} \mathrm{H}_{17} \mathrm{NO}_{3} \mathrm{Na}$ ) Calcd. : 246.1101, Found: 246.1102, Error: 0.4 ppm; IR $1646.0,1714.5 \mathrm{~cm}^{-1}$. 
A Tandem Semipinacol/Schmidt Reaction Leading to Versatile and Efficient Approach to

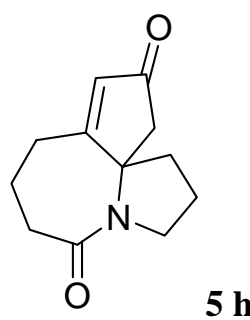

4h (45 mg, $0.20 \mathrm{mmol})$ was treated with $\mathrm{KO}{ }^{\mathrm{t}} \mathrm{Bu}(28 \mathrm{mg}, 0.24 \mathrm{mmol})$ in $2 \mathrm{ml}$ of ${ }^{\mathrm{t}} \mathrm{BuOH}$ at $40^{\circ} \mathrm{C}$. After 30 min, water diluted and extracted with $\mathrm{CH}_{2} \mathrm{Cl}_{2}$, dried over over $\mathrm{Na}_{2} \mathrm{SO}_{4}$, concentrated, and chromatographed to give the tricyclic lactam $5 \mathbf{h}\left(28 \mathrm{mg}, 68 \%\right.$ yield): ${ }^{1} \mathrm{H}$ NMR $\left(300 \mathrm{MHz}, \mathrm{CDCl}_{3}\right)$ $\delta \quad 1.61 \sim 1.67(\mathrm{~m}, 1 \mathrm{H}), 1.84 \sim 1.99(\mathrm{~m}, 2 \mathrm{H}), 2.00 \sim 2.11(\mathrm{~m}, 3 \mathrm{H}), 2.15 \sim 2.58(\mathrm{~m}, 5 \mathrm{H}), 2.69(\mathrm{~s}, 2 \mathrm{H})$, $2.82(\mathrm{dd}, 1 \mathrm{H}, \mathrm{J}=12.0 \mathrm{~Hz}, 8.7 \mathrm{~Hz}), 3.55 \sim 3.71(\mathrm{~m}, 2 \mathrm{H}), 6.01(\mathrm{~s}, 1 \mathrm{H}) ;{ }^{13} \mathrm{C} \mathrm{NMR}\left(75 \mathrm{MHz}, \mathrm{CDCl}_{3}\right) \delta$ 21.4, 24.2, 25.2, 32.5, 40.9, 47.0, 50.3, 70.7, 129.2, 170.8, 180.2, 202.7; MS: 205, 150, 134, 122; HMRS (ESI, $\mathrm{M}^{+}+\mathrm{H}, \mathrm{C}_{12} \mathrm{H}_{16} \mathrm{NO}_{2}$ ) Calcd. : 206.1176, Found: 206.1177, Error: 0.5 ppm; IR 1639.3, $1712.1 \mathrm{~cm}^{-1}$.

\section{References:}

(1) Miwako M.; Yumiko W.; Takao U.; Kagari Y.; Katsumi C.; Yoshio B., J. Org. Chem. 1983, 48, 4058-4067.

(2) Milligan, G.; Mossman C. J.; Aubé, J. J. Am. Chem. Soc. 1995, 117, 10449-10459. 
A Tandem Semipinacol/Schmidt Reaction Leading to Versatile and Efficient Approach to Azaquaternary Alkaloid Skeletons Peiming Gu, Yu-Ming Zhao, Yong Qiang Tu, ${ }^{*}$ Yufei Ma, and Fumin Zhang

X-Ray El I i psoi d PI ot s of $2 \mathrm{~h}$ and $2 \mathrm{i}$ have been deposi ted at the Canbr i dge Cryst al I ogr aphi $\mathrm{c}$ Dat a Centre and al l ocat ed the depositi on number s CCDC 616555 \& 616556<smiles>C=CC[C@]1(O)CCCN2O[C@@H]1CCCC2=O</smiles>

It is complicated of C10-C12 in the single crystalloid. It is explained that in the crystalloid two conformation exist with the ratio of about 3:1. And it does not influence the relative stereochemistry.

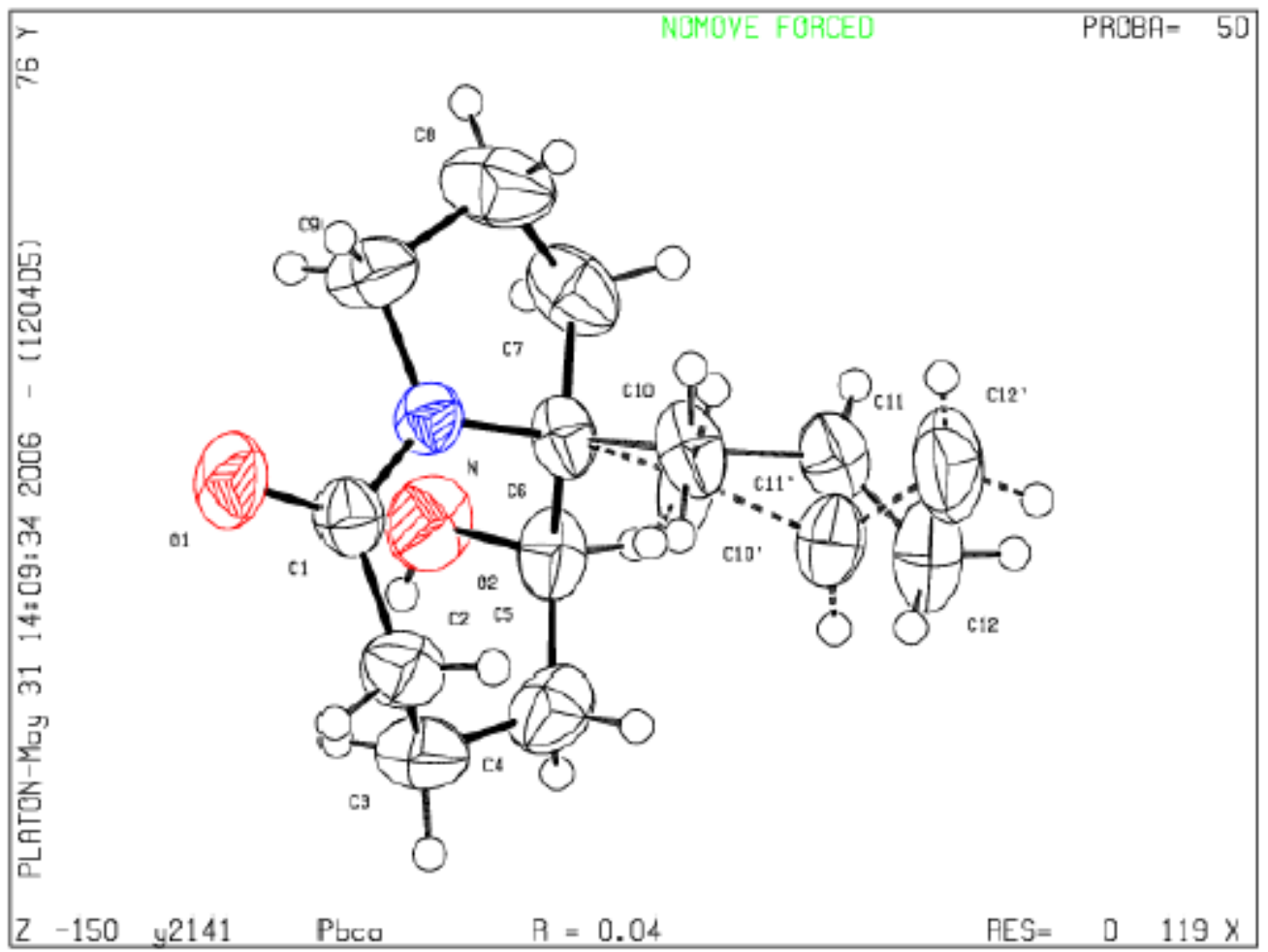


A Tandem Semipinacol/Schmidt Reaction Leading to Versatile and Efficient Approach to<smiles>O=C1CCC[C@]23CCC[C@@H](O)[C@H]2CCN13</smiles>

The major product $2 \mathrm{i} 1$ was confirmed by $\mathrm{X}$-ray crystallography analysis.

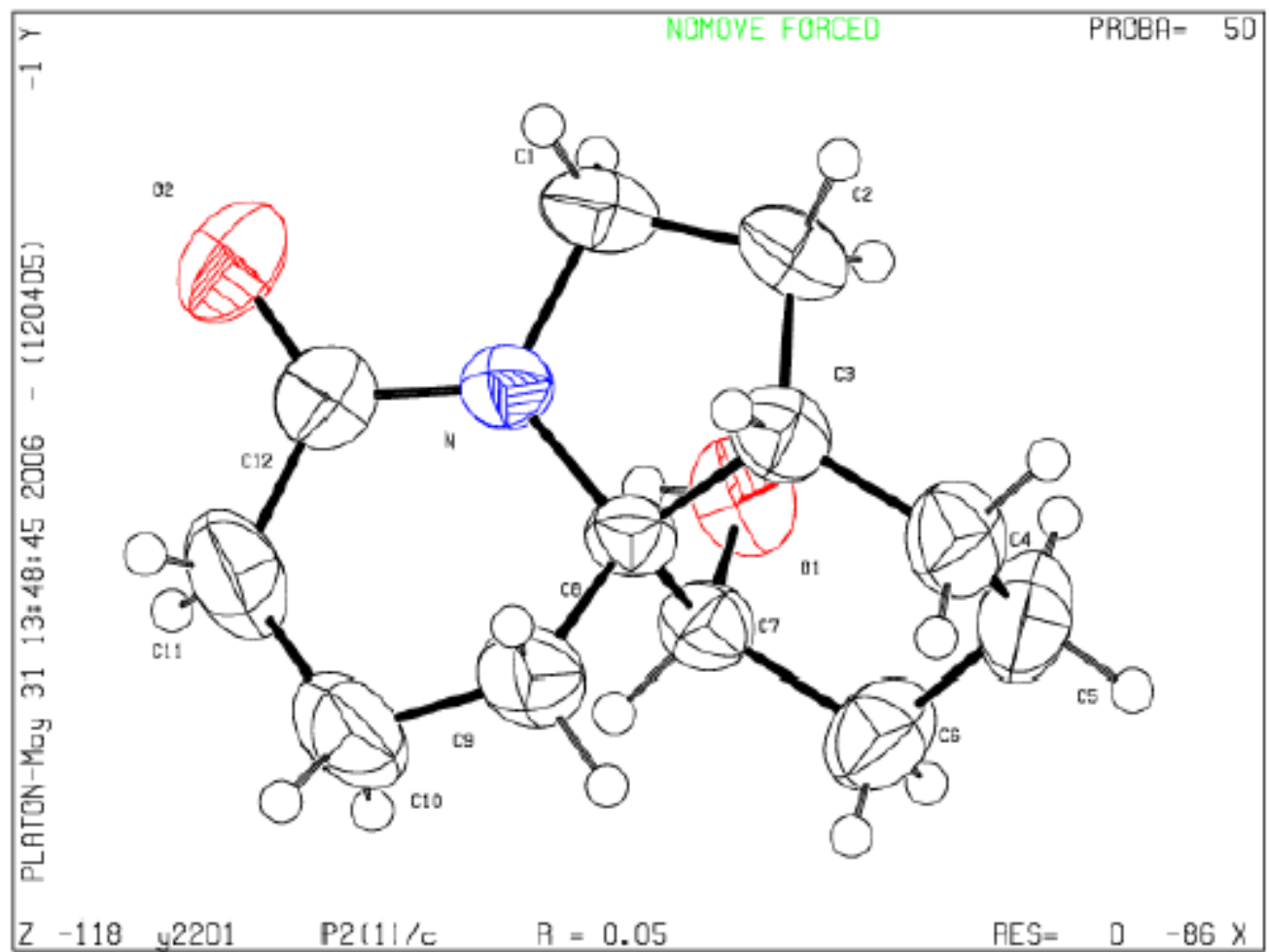


A Tandem Semipinacol/Schmidt Reaction Leading to Versatile and Efficient Approach to Azaquaternary Alkaloid Skeletons Peiming Gu, Yu-Ming Zhao, Yong Qiang Tu, ${ }^{*}$ Yufei Ma, and Fumin Zhang

\section{Copies of ${ }^{1} \mathrm{H}$ NMR and ${ }^{13} \mathrm{C}$ NMR of Substrates and Products}
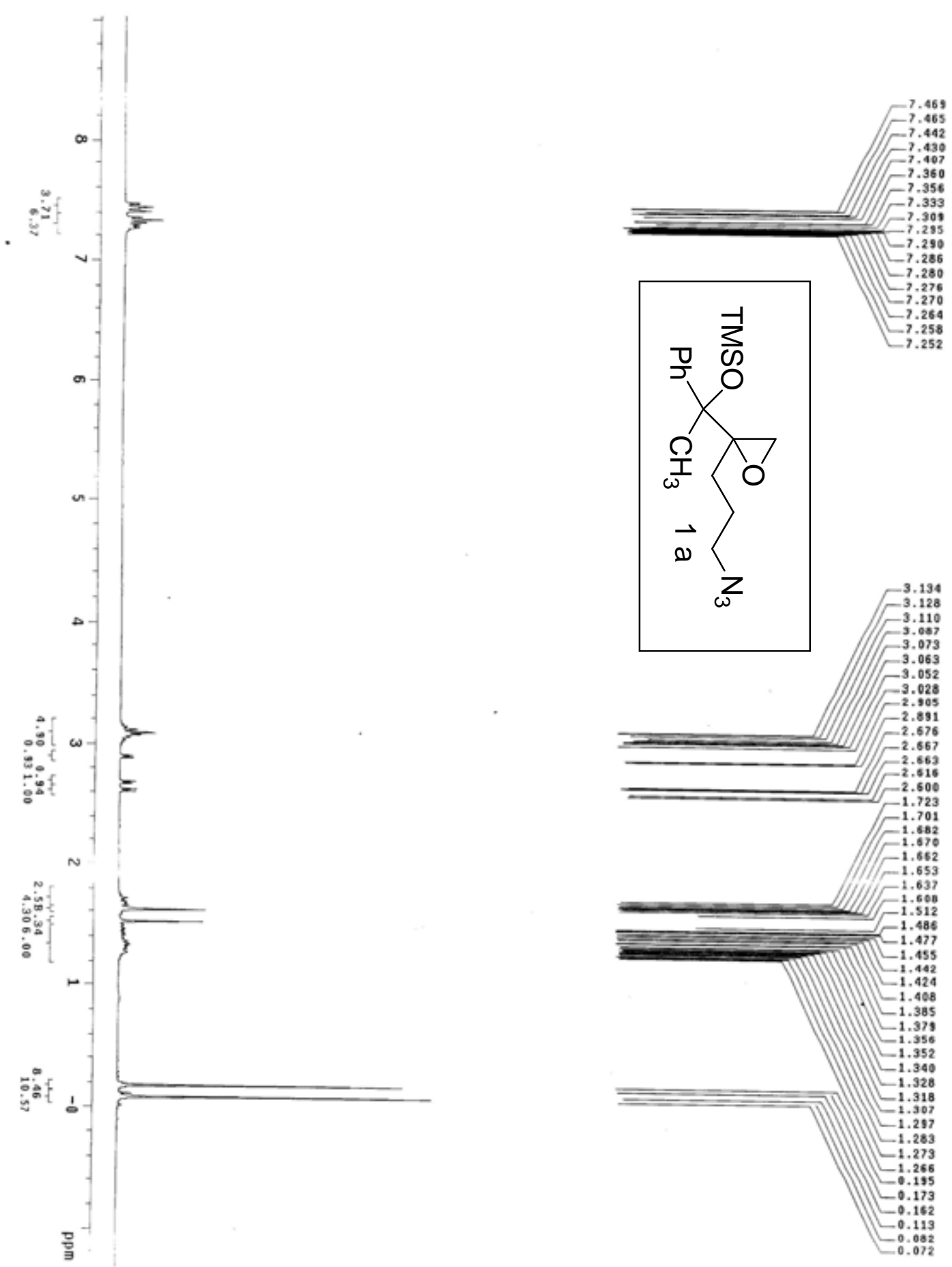
A Tandem Semipinacol/Schmidt Reaction Leading to Versatile and Efficient Approach to
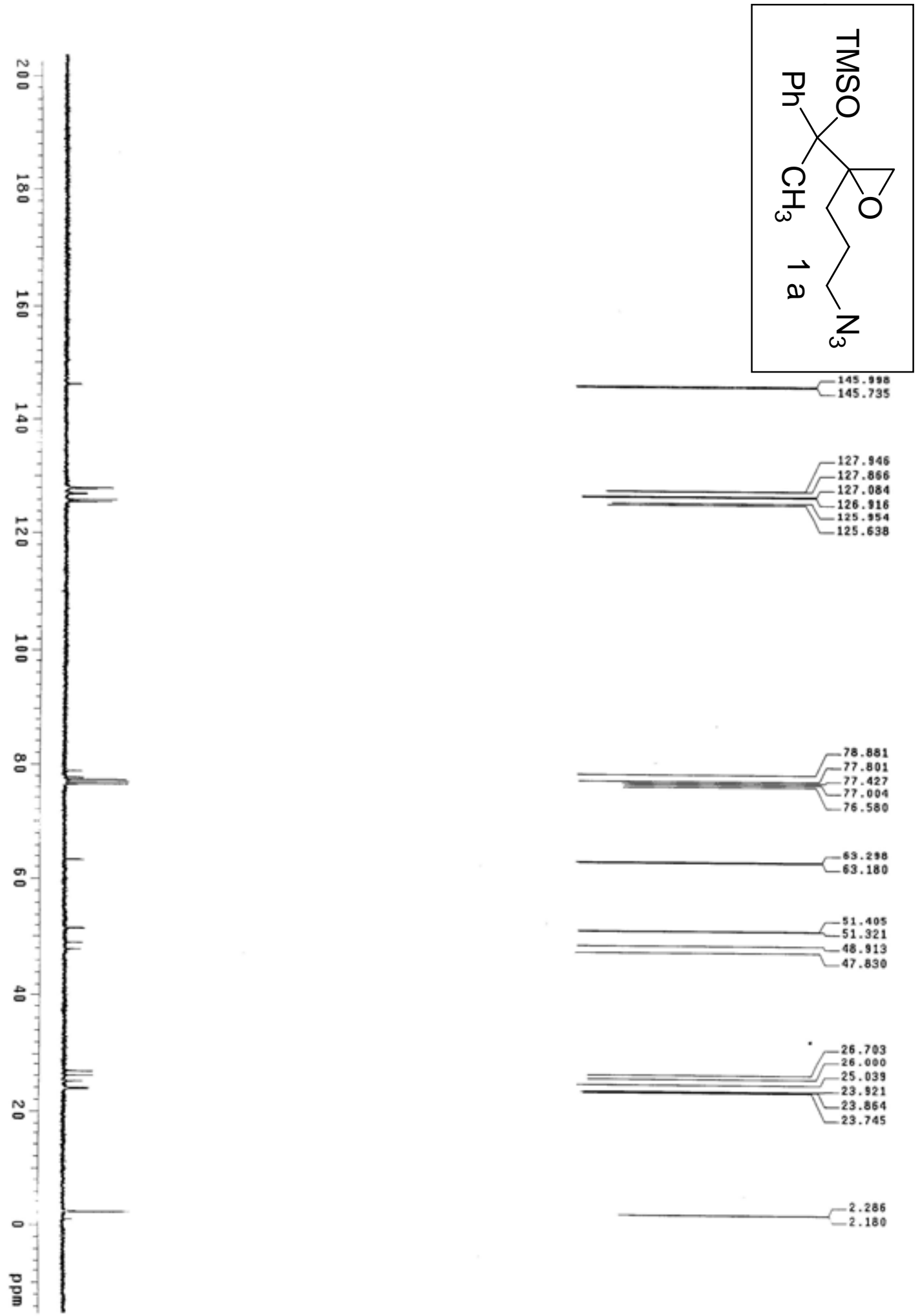

$-2.286$ 
A Tandem Semipinacol/Schmidt Reaction Leading to Versatile and Efficient Approach to
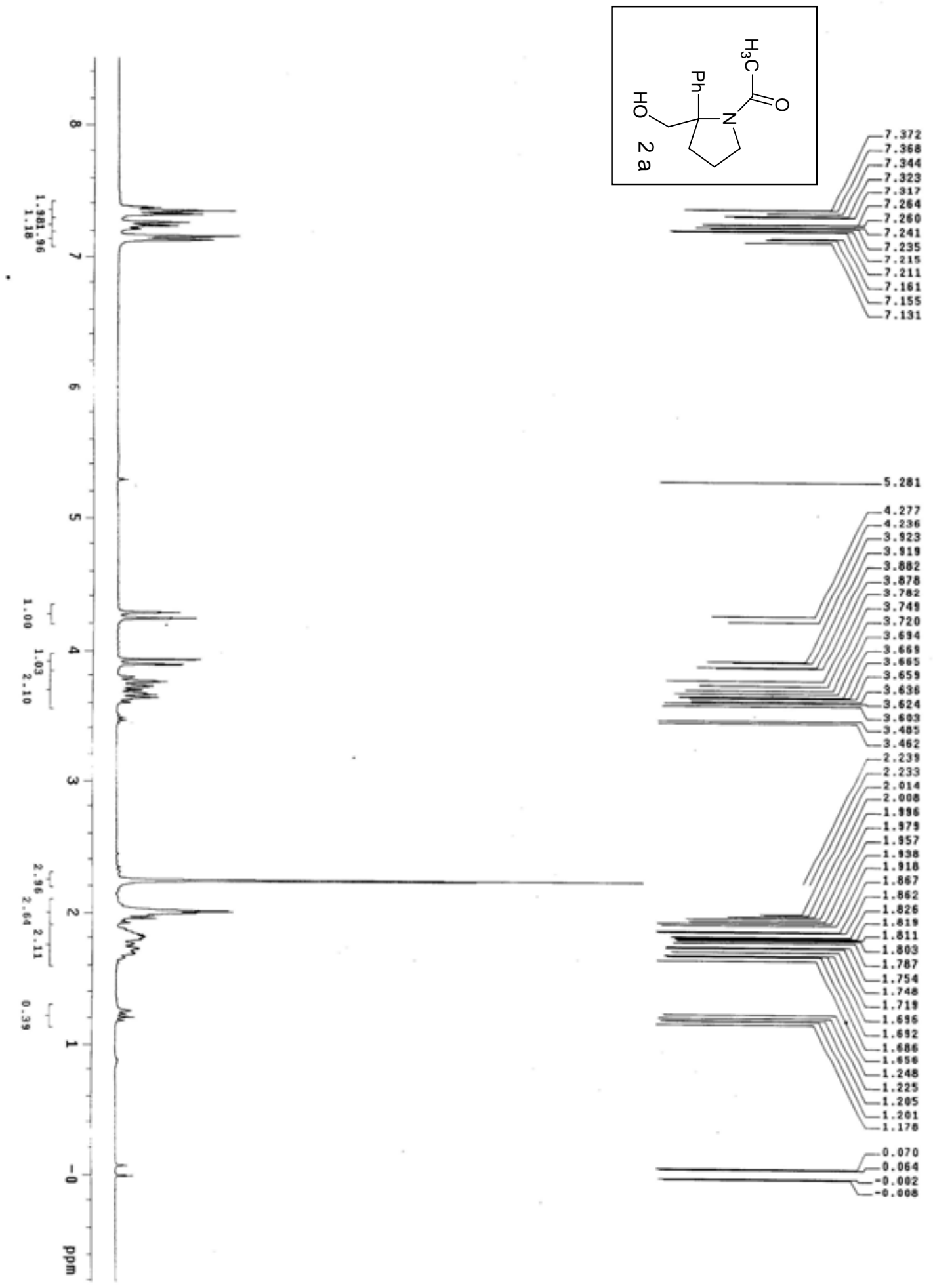
A Tandem Semipinacol/Schmidt Reaction Leading to Versatile and Efficient Approach to
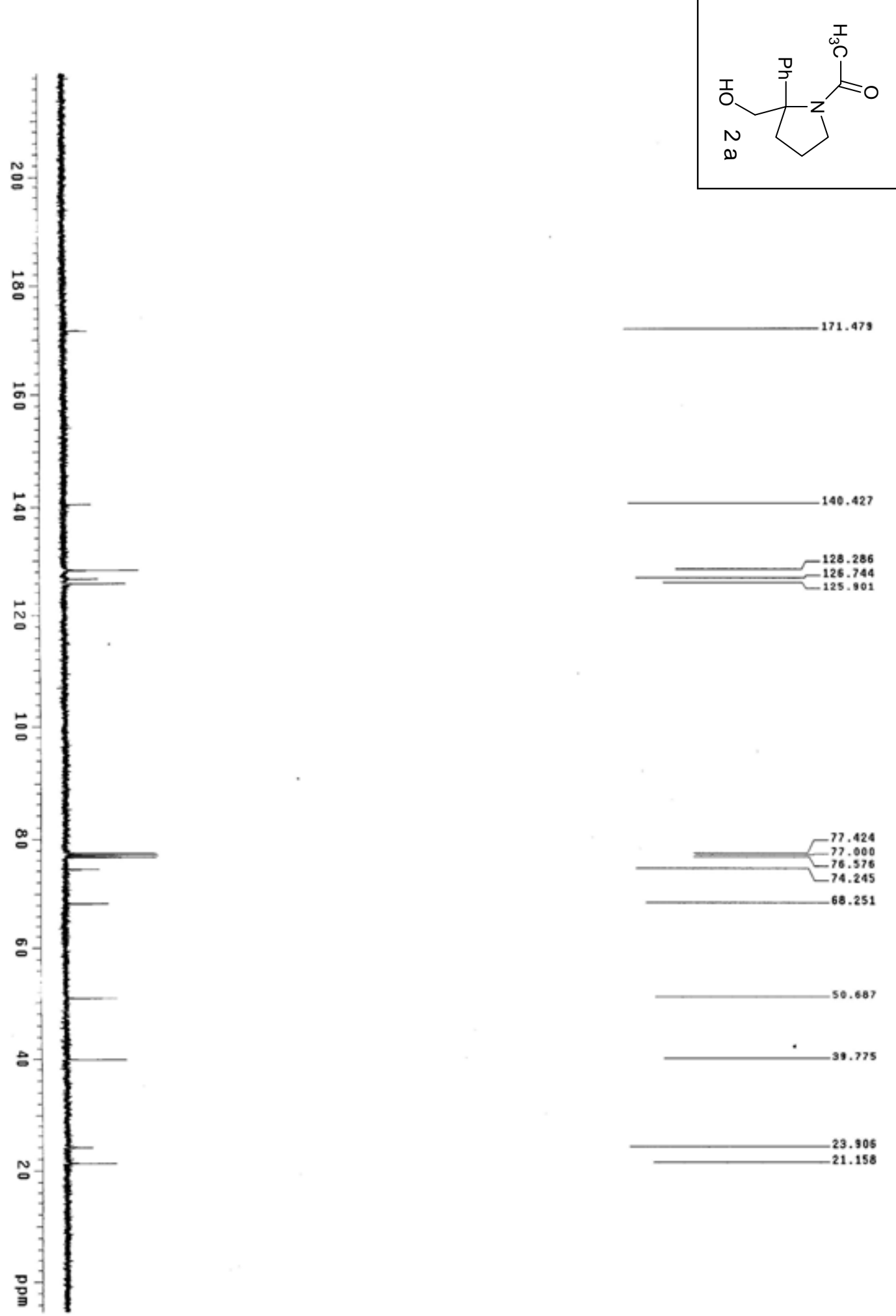

171.479
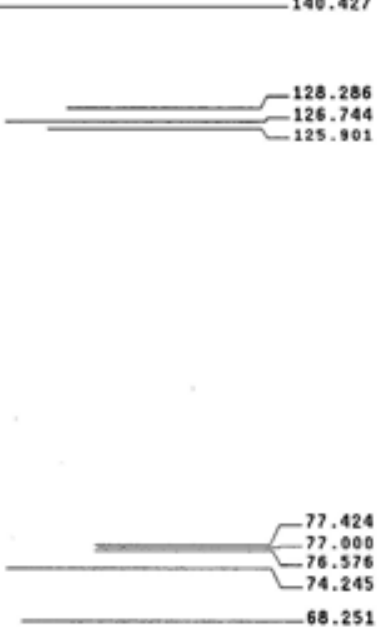

$-50.687$

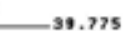

23.905

21.158 
A Tandem Semipinacol/Schmidt Reaction Leading to Versatile and Efficient Approach to

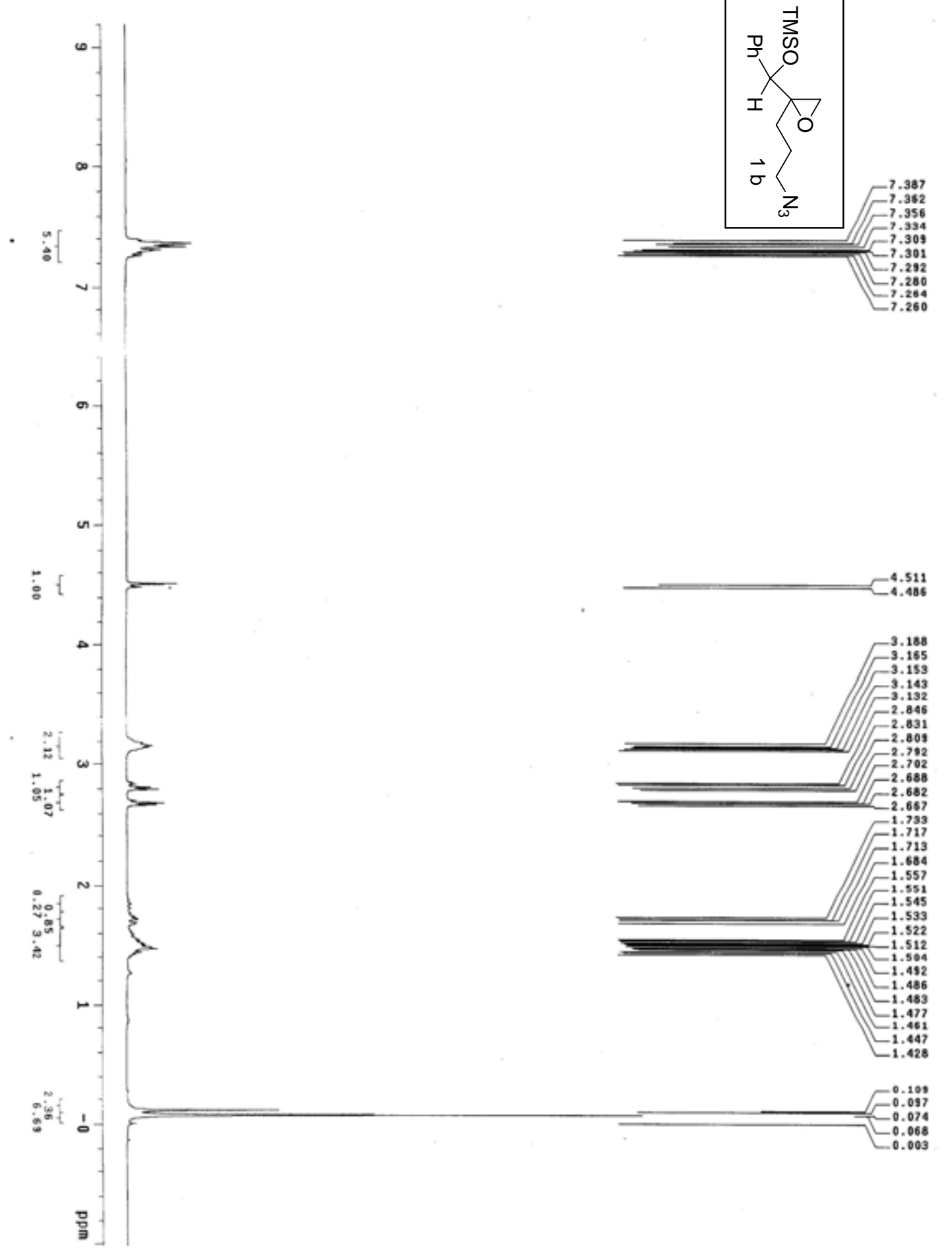


A Tandem Semipinacol/Schmidt Reaction Leading to Versatile and Efficient Approach to
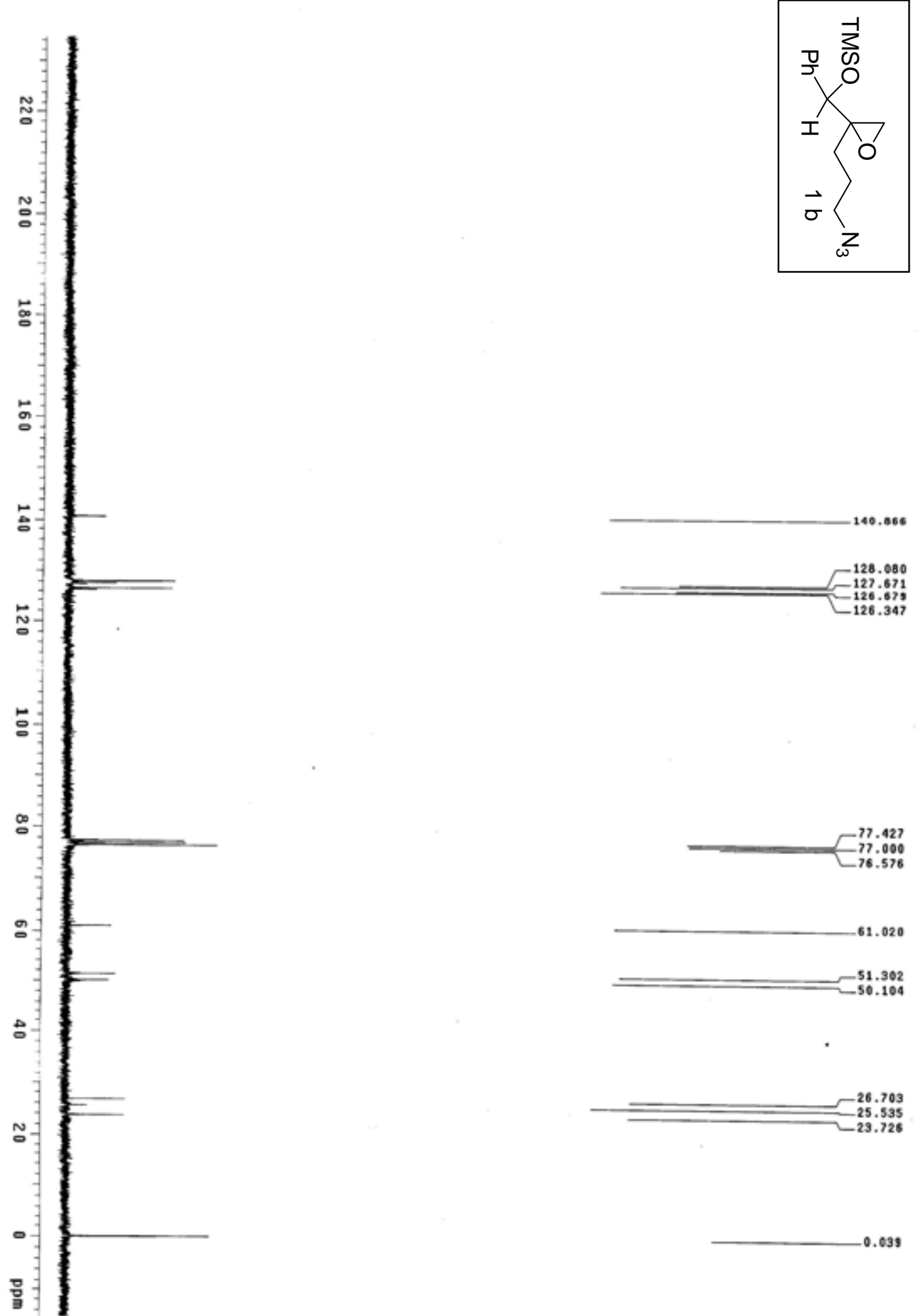
A Tandem Semipinacol/Schmidt Reaction Leading to Versatile and Efficient Approach to
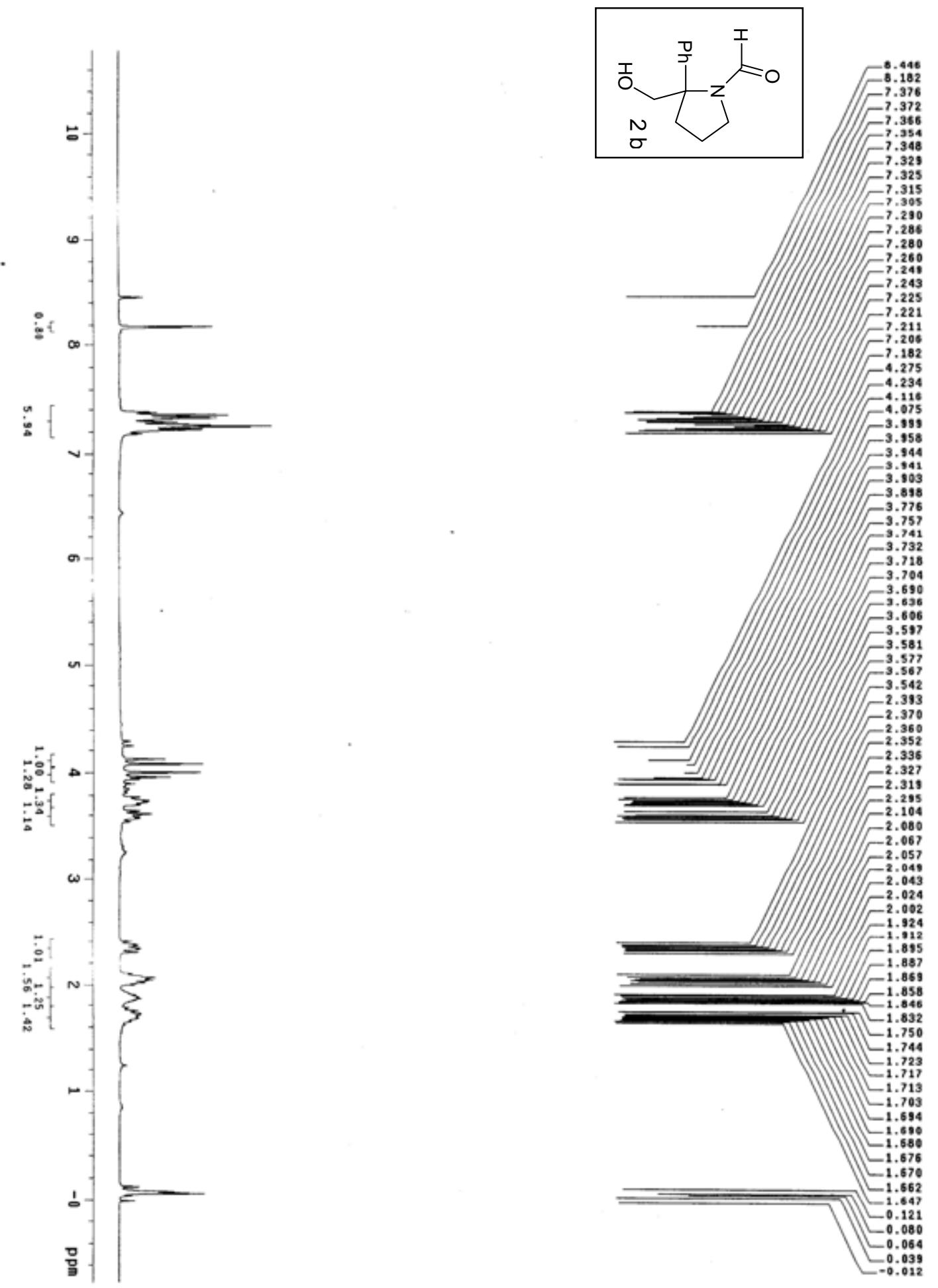
A Tandem Semipinacol/Schmidt Reaction Leading to Versatile and Efficient Approach to
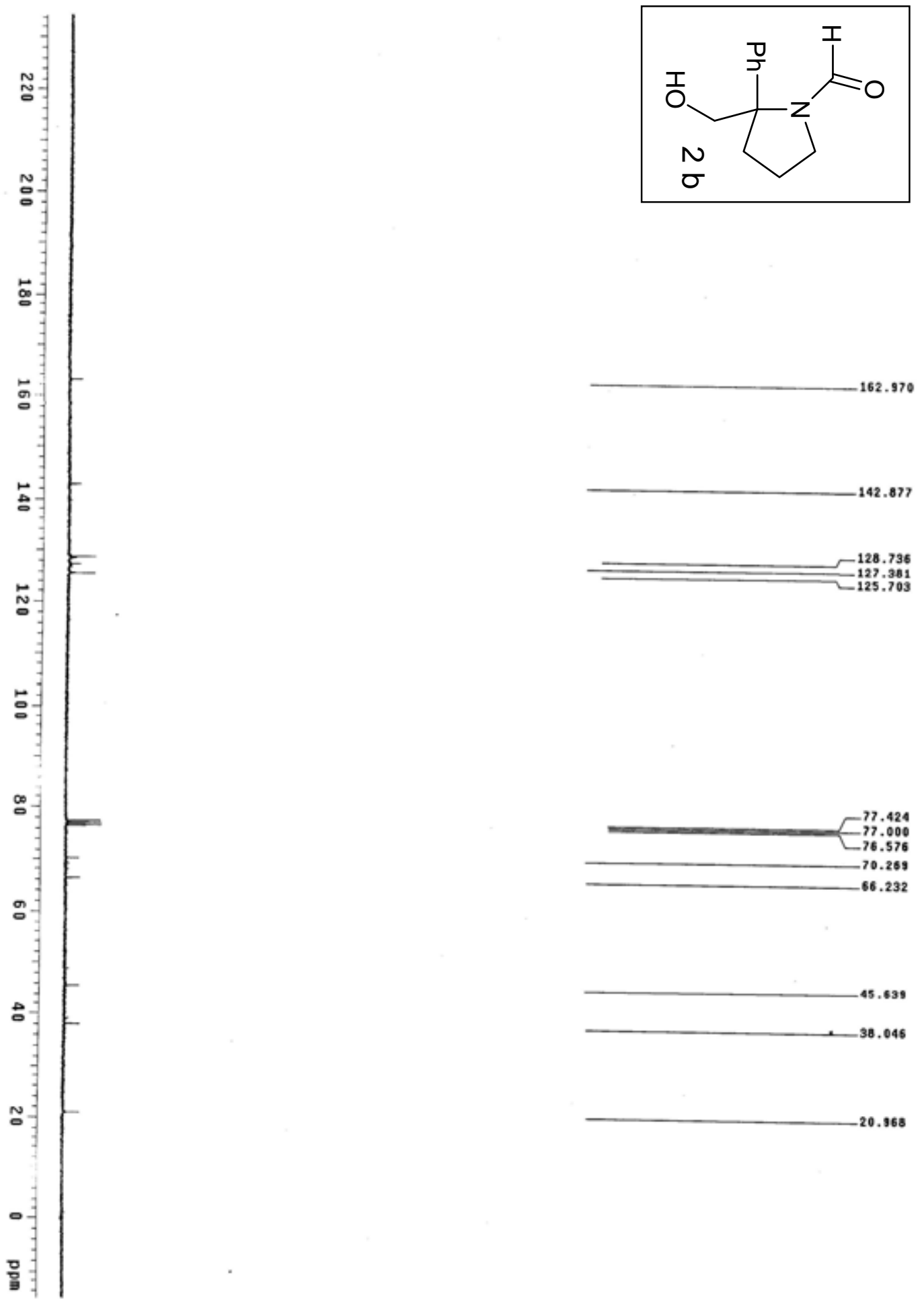

$-20.968$ 
A Tandem Semipinacol/Schmidt Reaction Leading to Versatile and Efficient Approach to

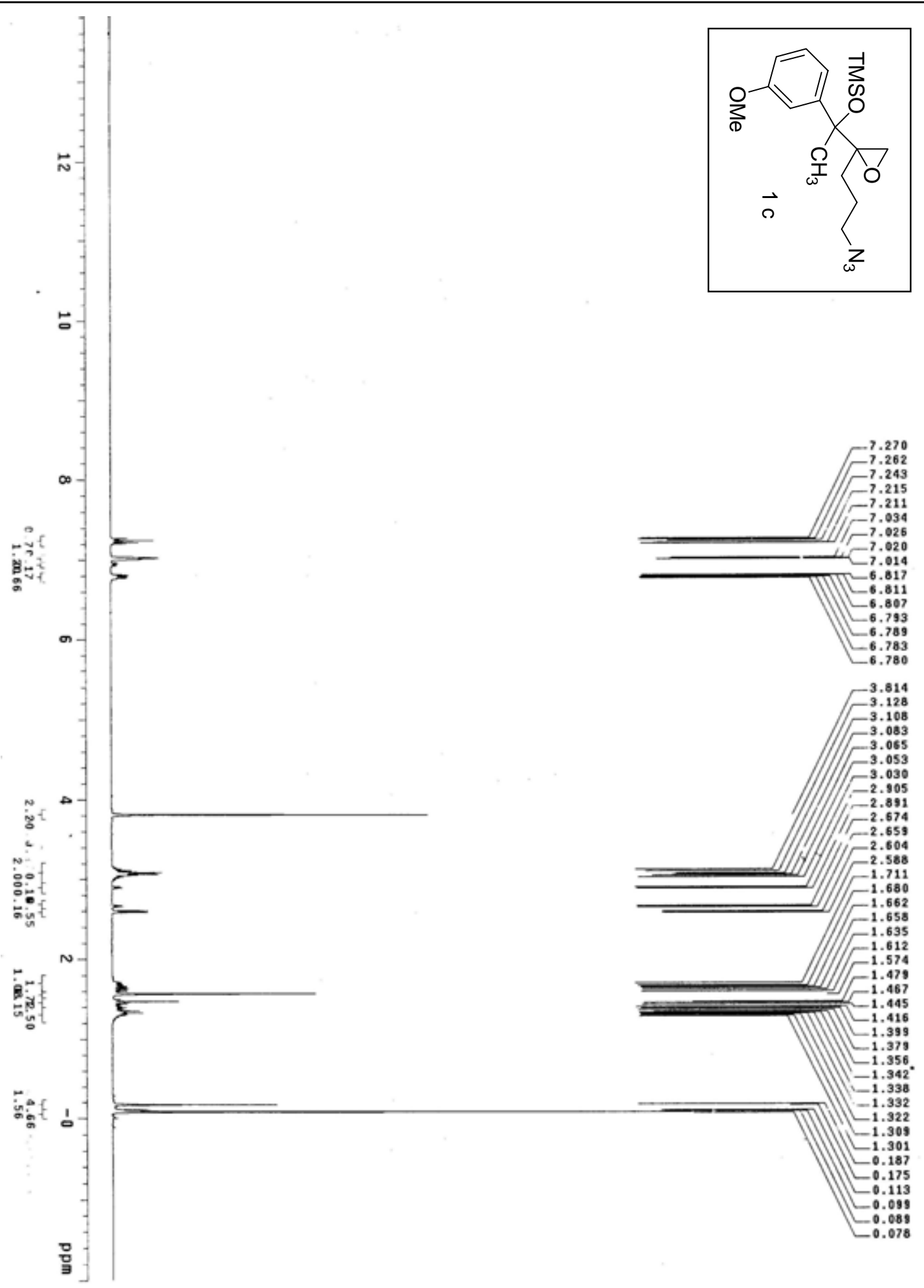


A Tandem Semipinacol/Schmidt Reaction Leading to Versatile and Efficient Approach to
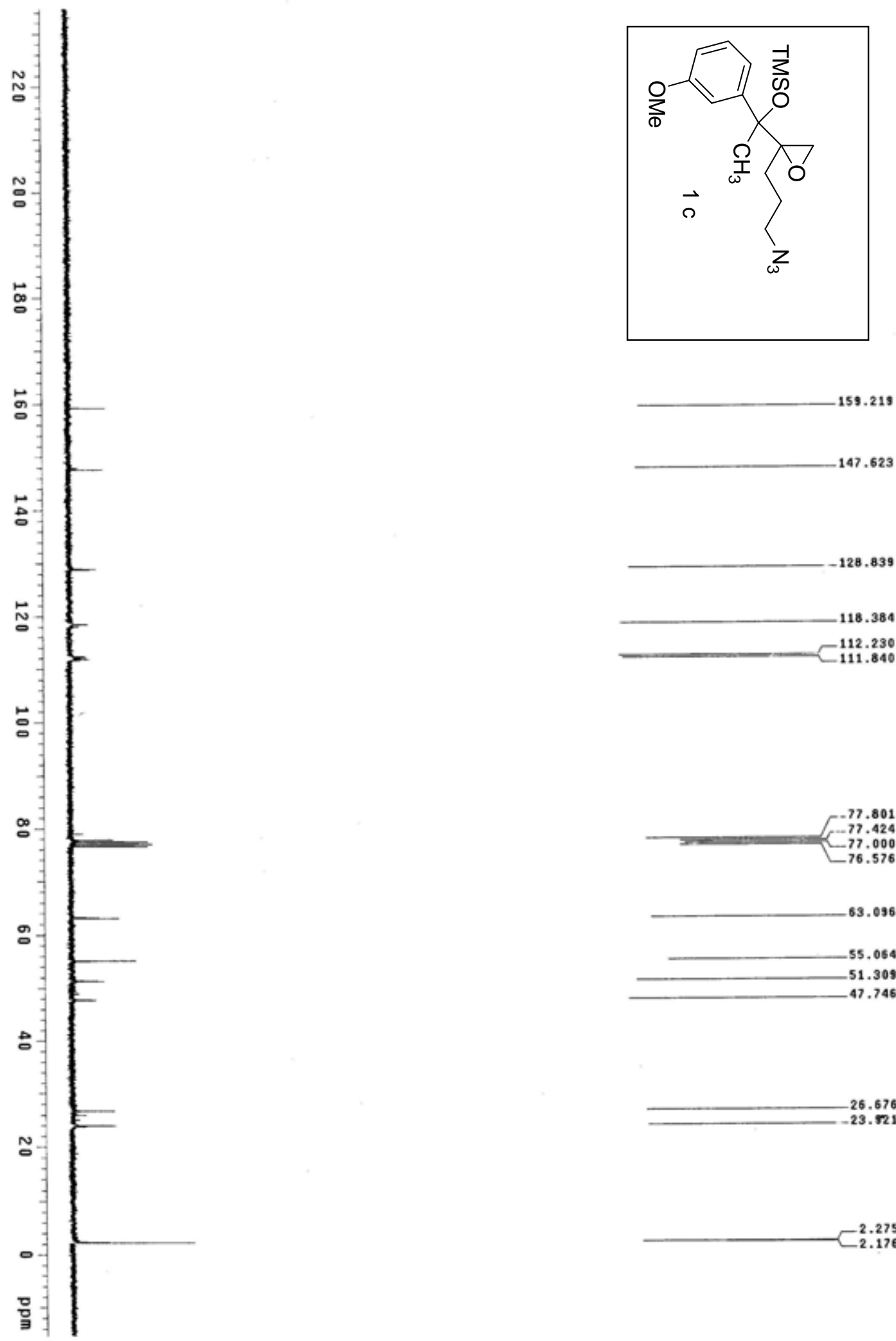

$-147.623$

128.839

18.384

112.230
111.840

26.676

-77.801
-77.424

$-77.424$

77.000
-76.576

$-63.096$

55.054

51.309

$-47.746$

$-23.821$ 
A Tandem Semipinacol/Schmidt Reaction Leading to Versatile and Efficient Approach to

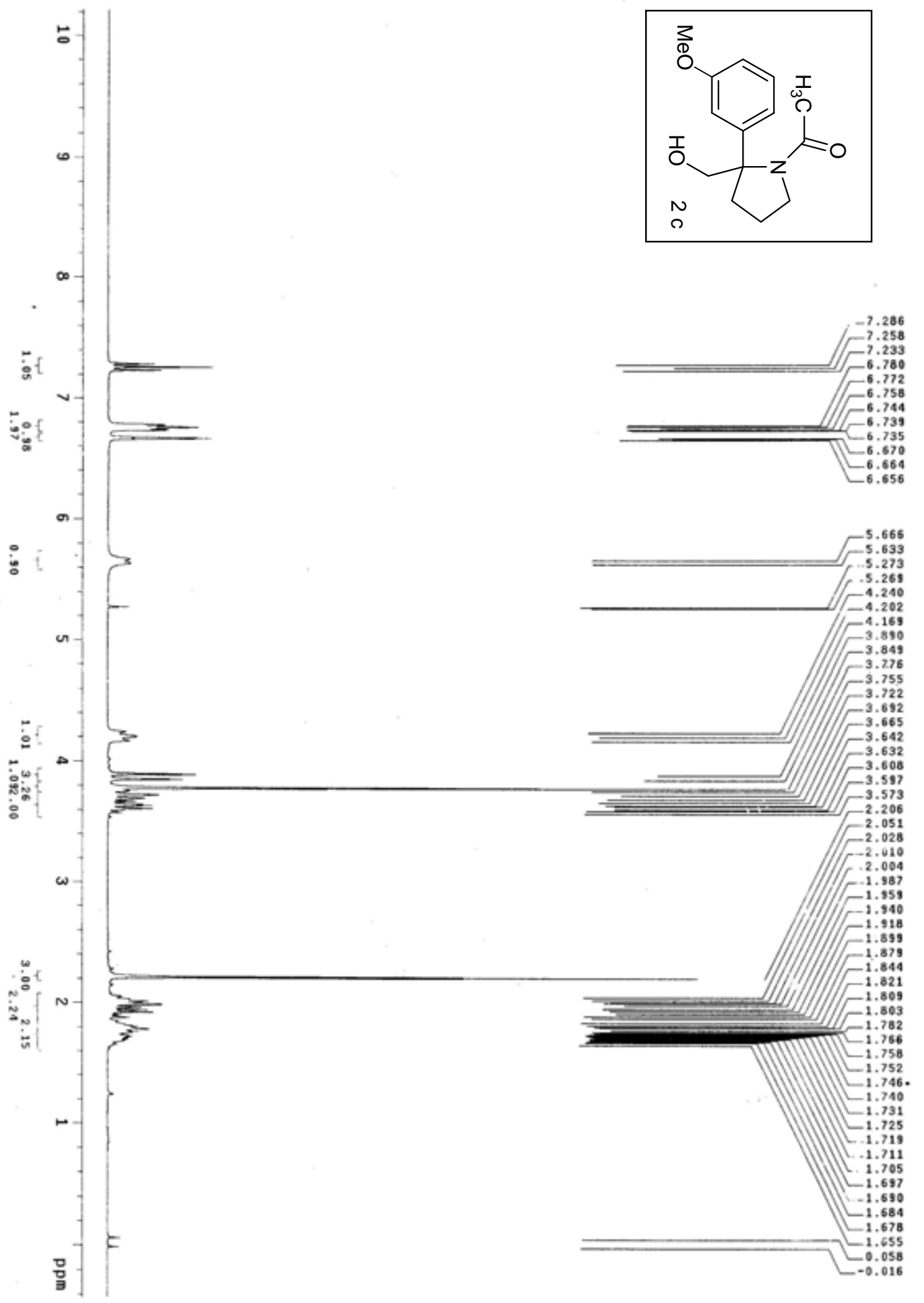


A Tandem Semipinacol/Schmidt Reaction Leading to Versatile and Efficient Approach to
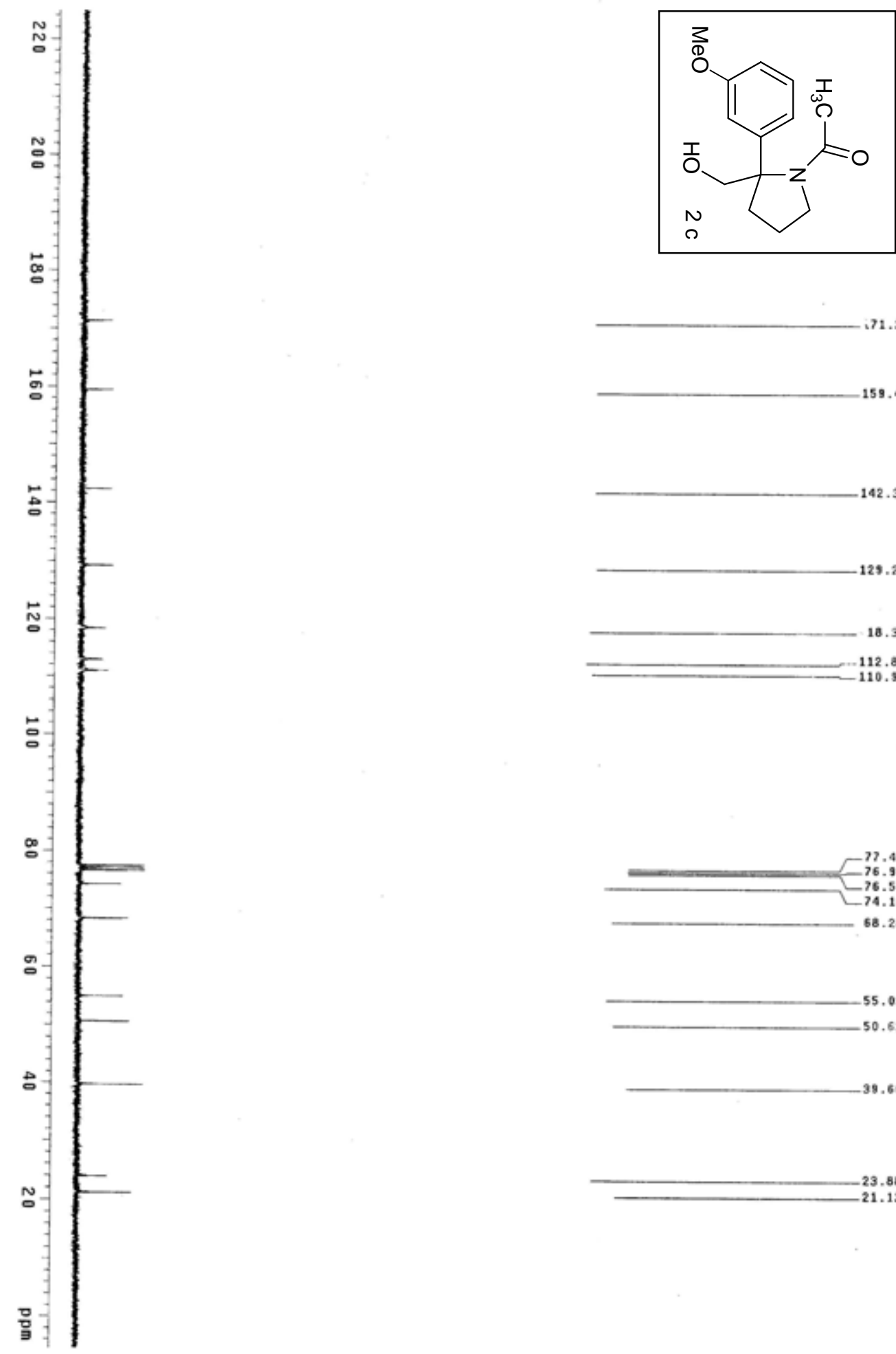

159.444

142.358

$-129.253$

18.377

112.859

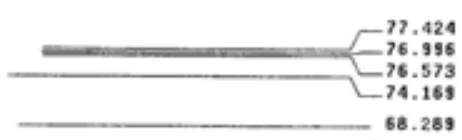

5,037

$-50.630$

$-39.664$

$-23.883$

$-21.139$ 
A Tandem Semipinacol/Schmidt Reaction Leading to Versatile and Efficient Approach to

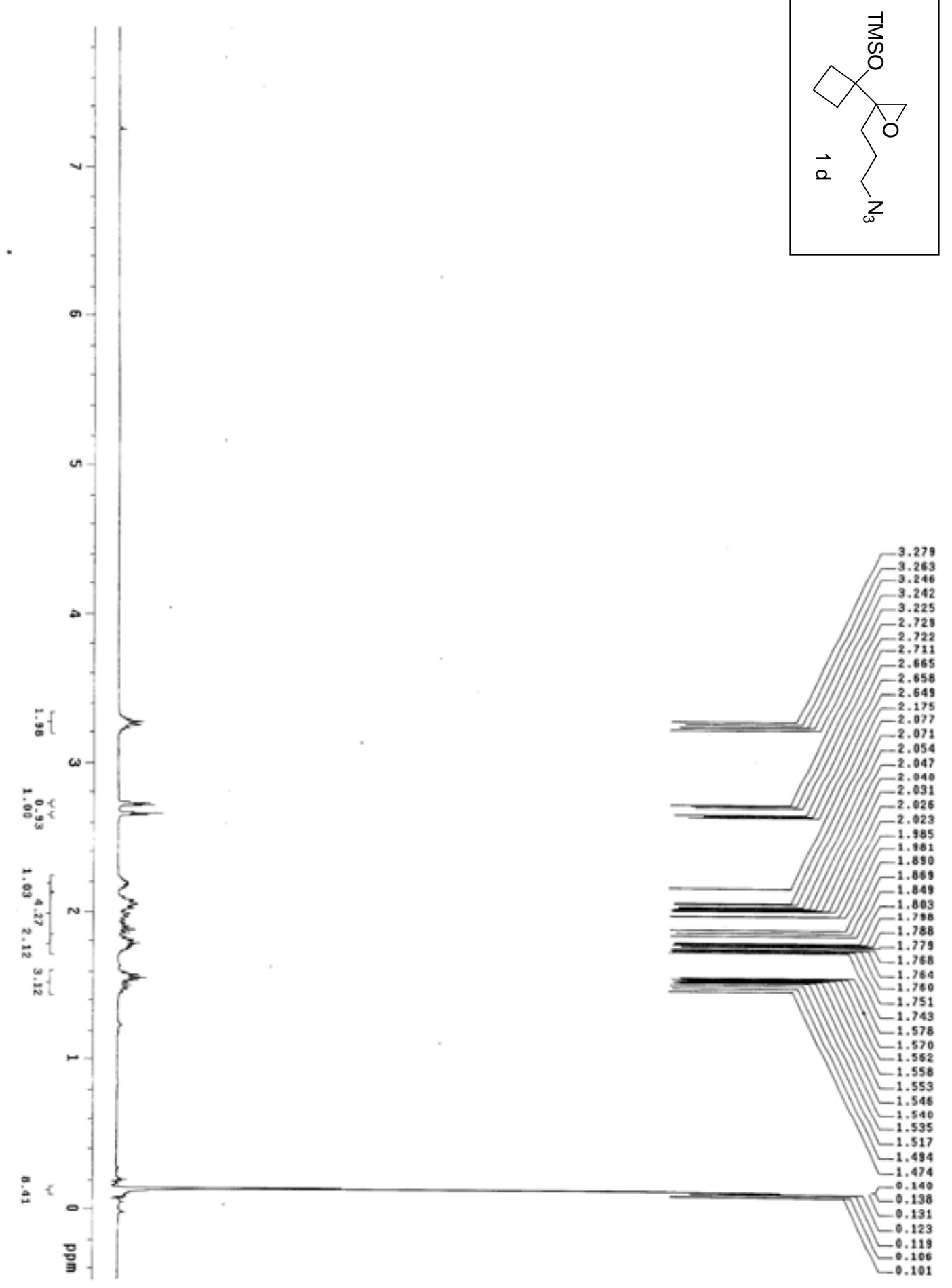


A Tandem Semipinacol/Schmidt Reaction Leading to Versatile and Efficient Approach to
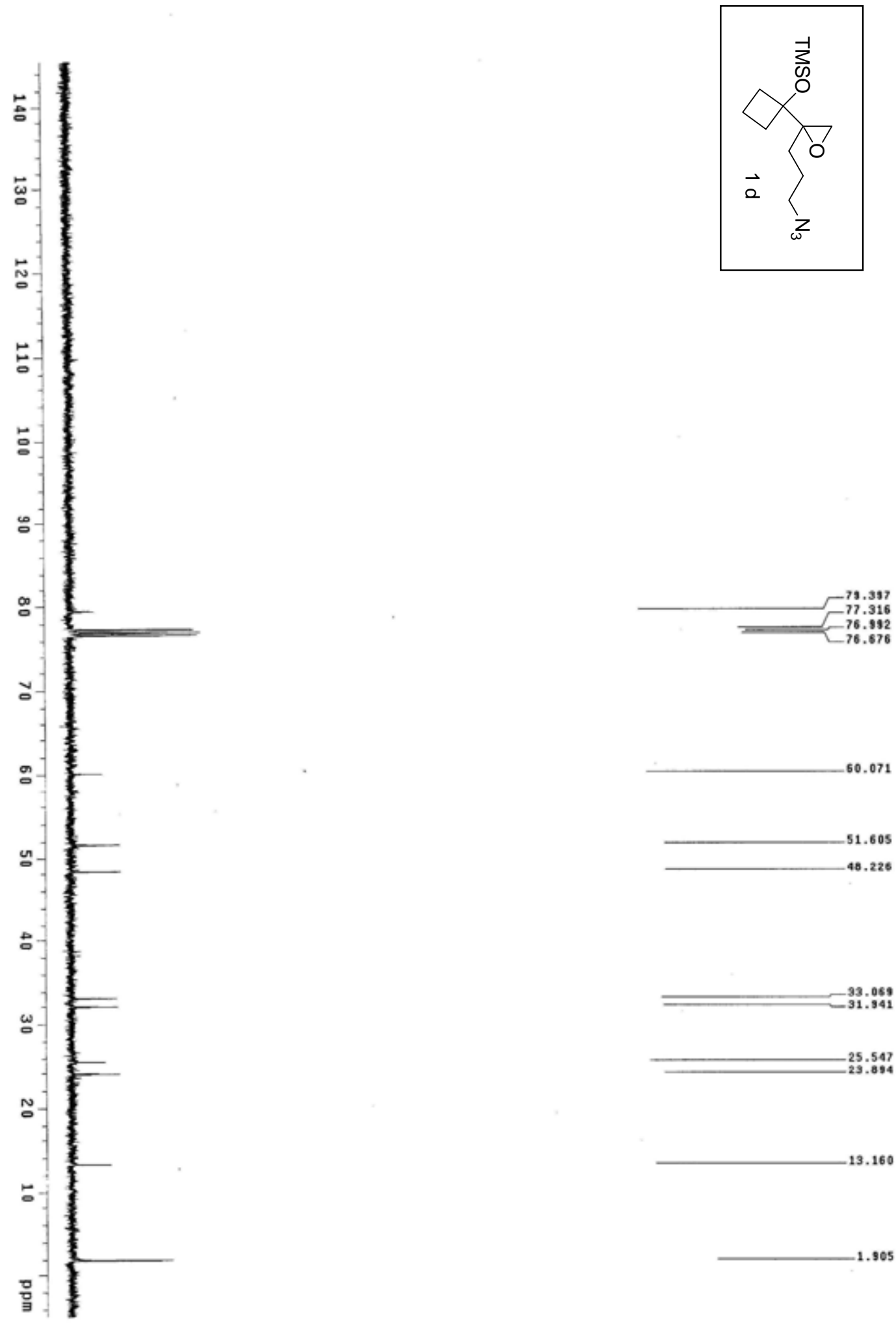

51.605

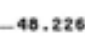


A Tandem Semipinacol/Schmidt Reaction Leading to Versatile and Efficient Approach to
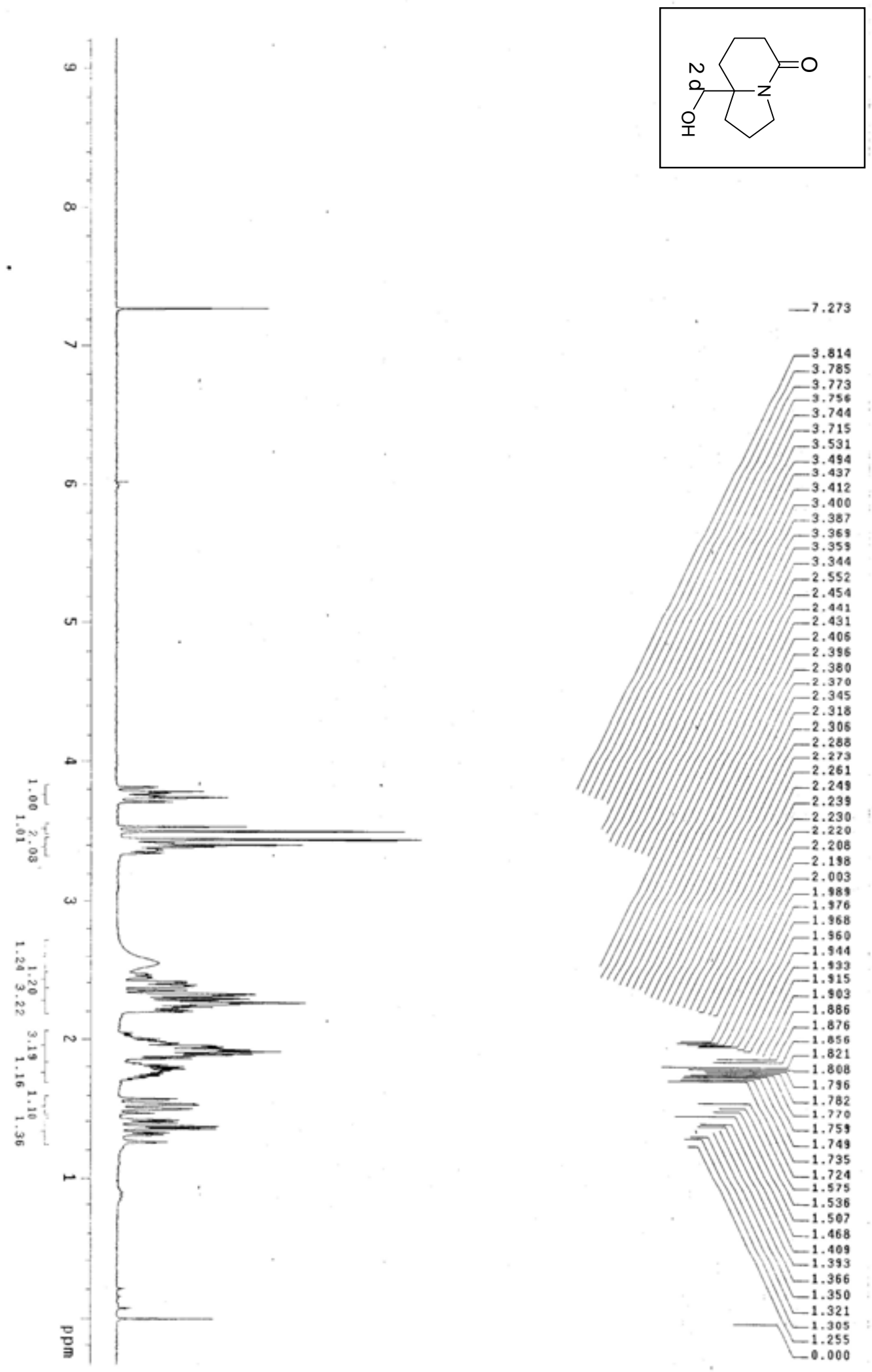
A Tandem Semipinacol/Schmidt Reaction Leading to Versatile and Efficient Approach to
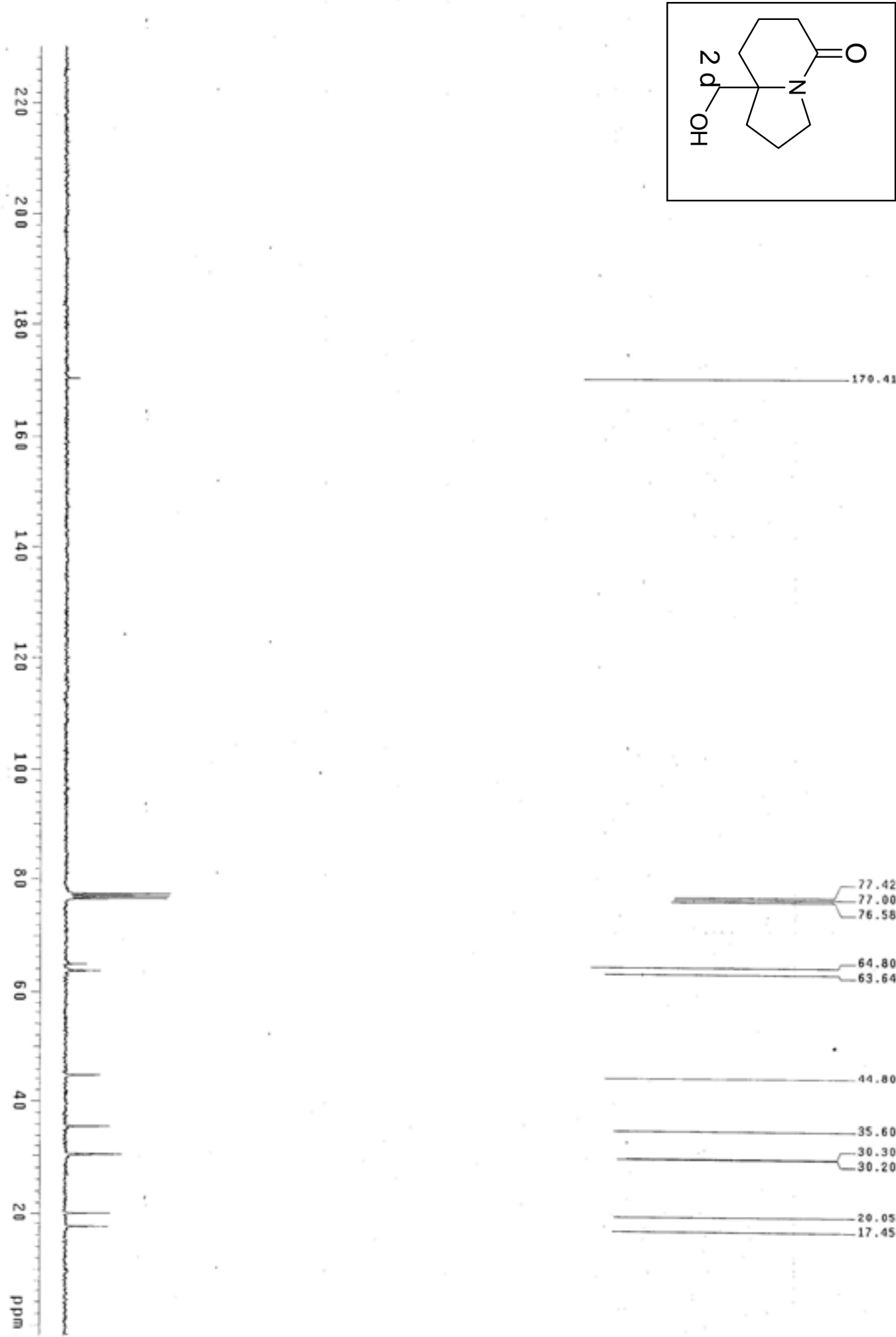
A Tandem Semipinacol/Schmidt Reaction Leading to Versatile and Efficient Approach to
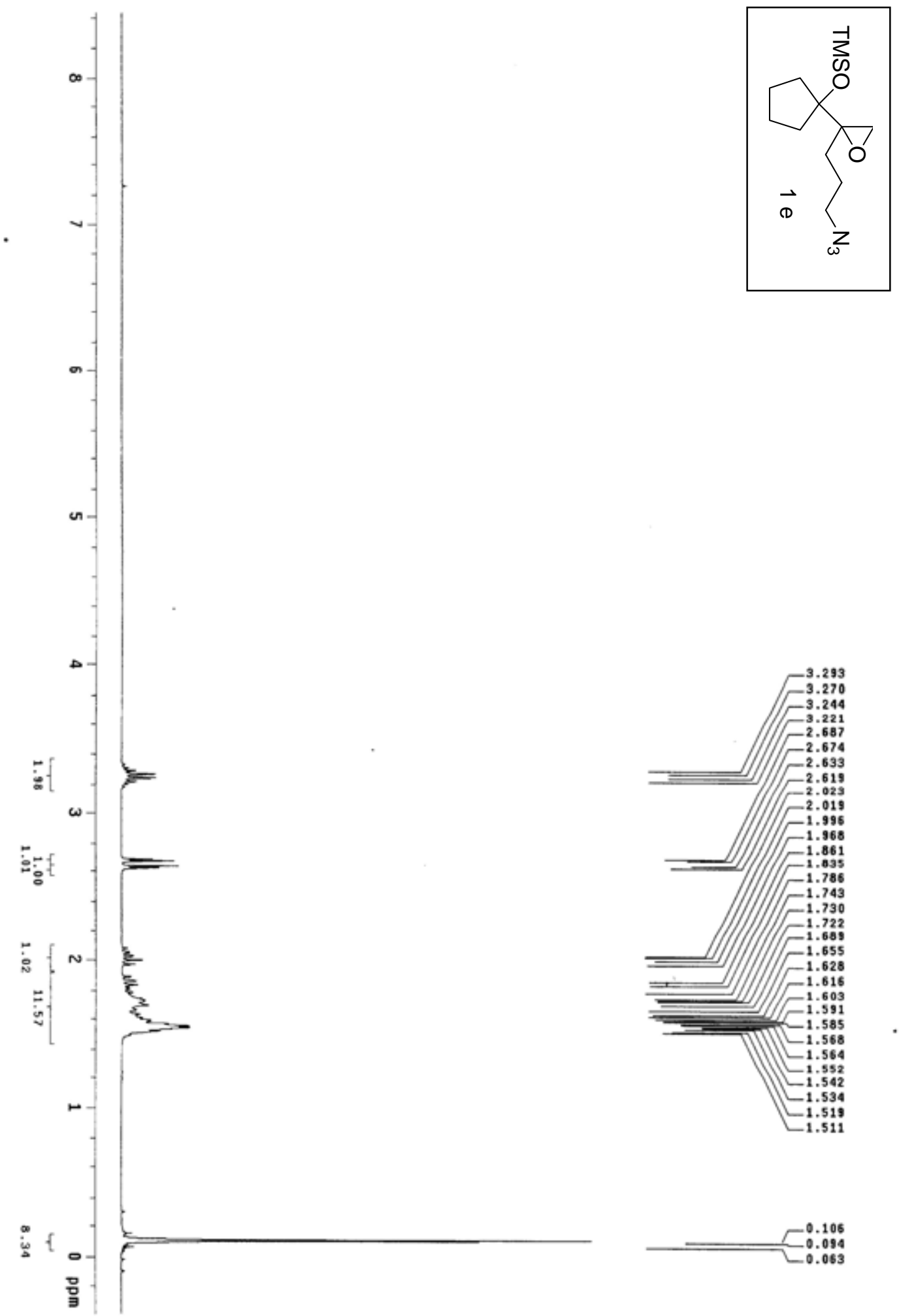
A Tandem Semipinacol/Schmidt Reaction Leading to Versatile and Efficient Approach to
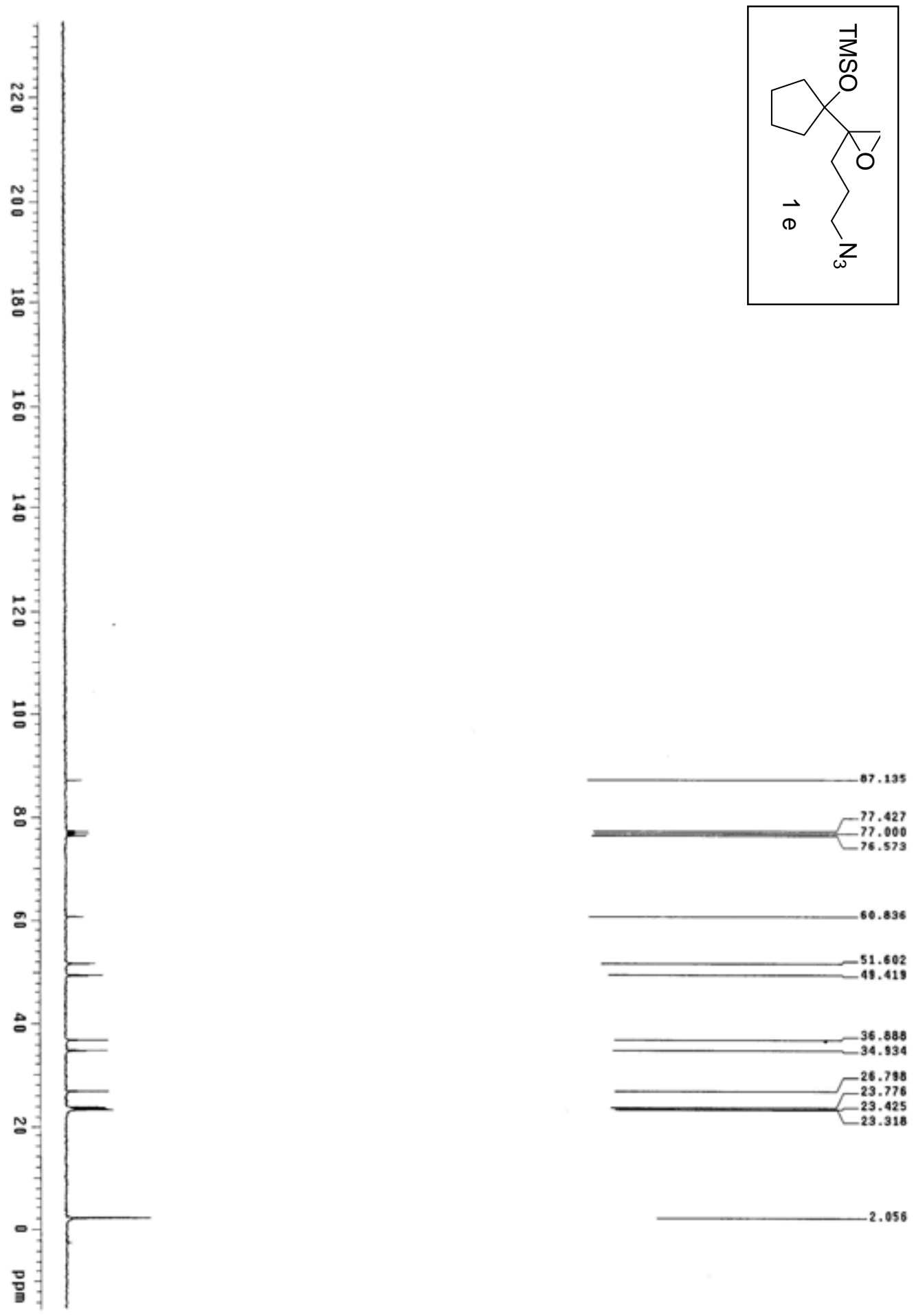

1.602

49.419

-36.888
-34.934

$-26.798$

$-23.776$

-23.425
-2318

$-2,056$ 
A Tandem Semipinacol/Schmidt Reaction Leading to Versatile and Efficient Approach to
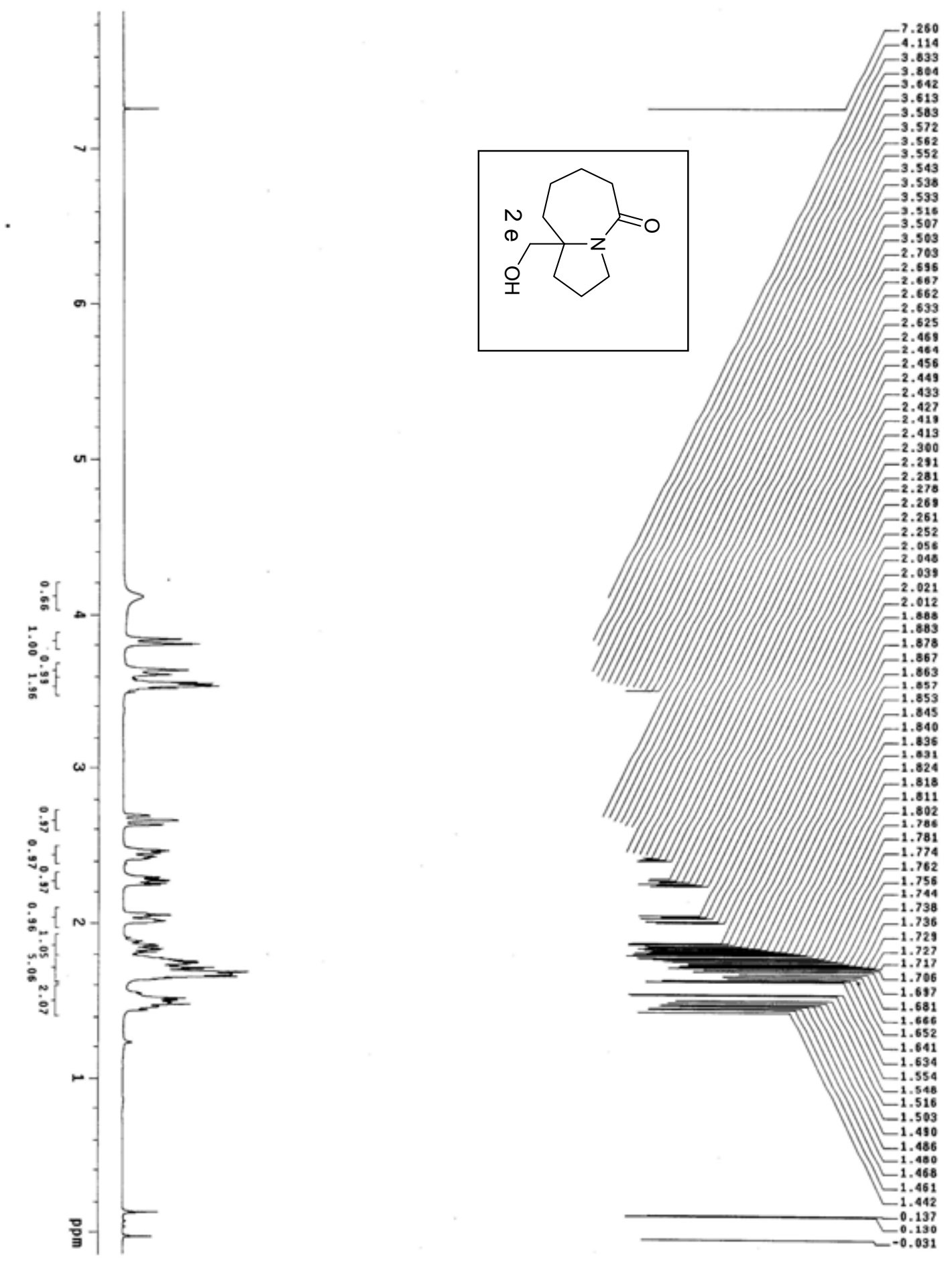
A Tandem Semipinacol/Schmidt Reaction Leading to Versatile and Efficient Approach to
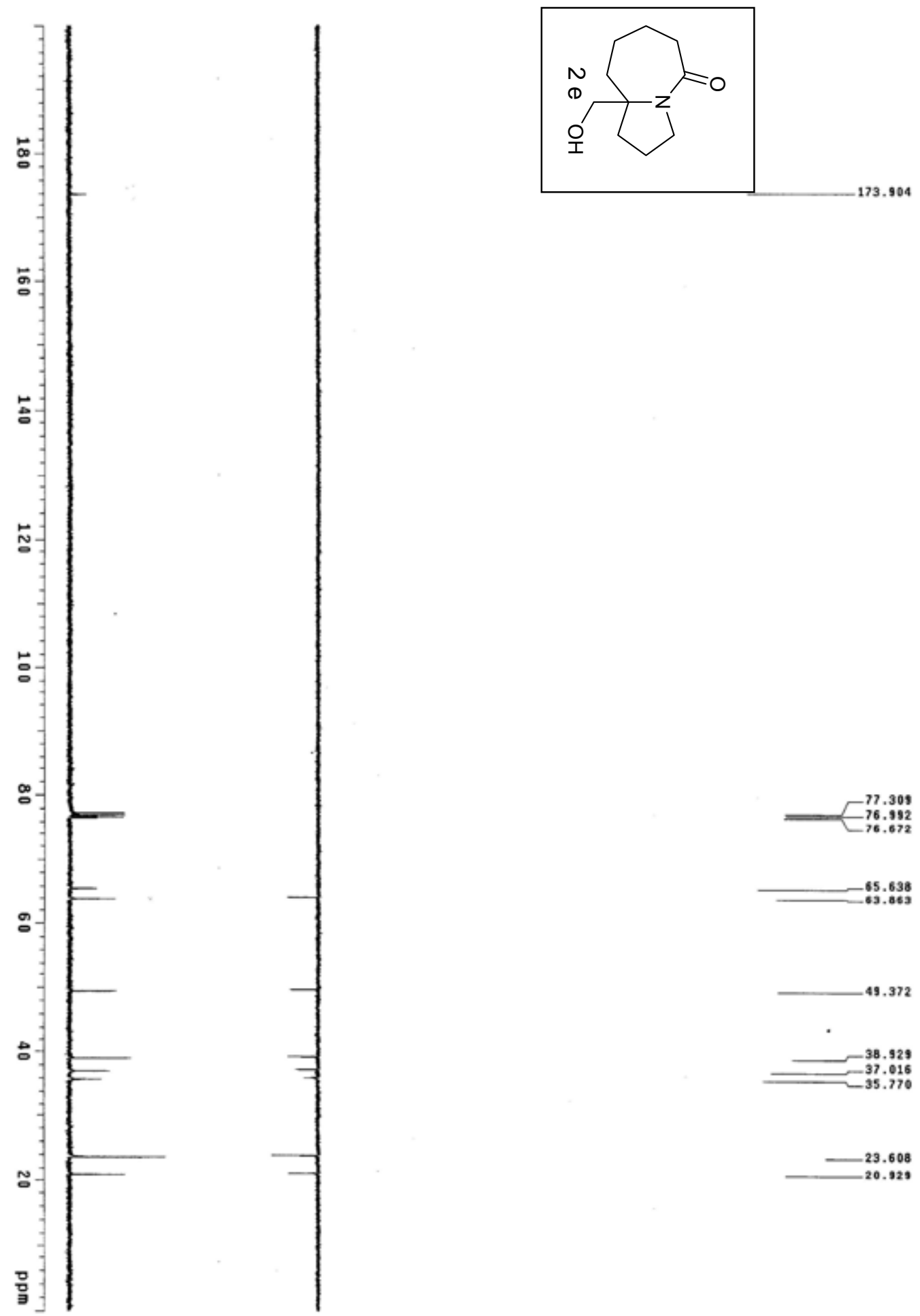
A Tandem Semipinacol/Schmidt Reaction Leading to Versatile and Efficient Approach to
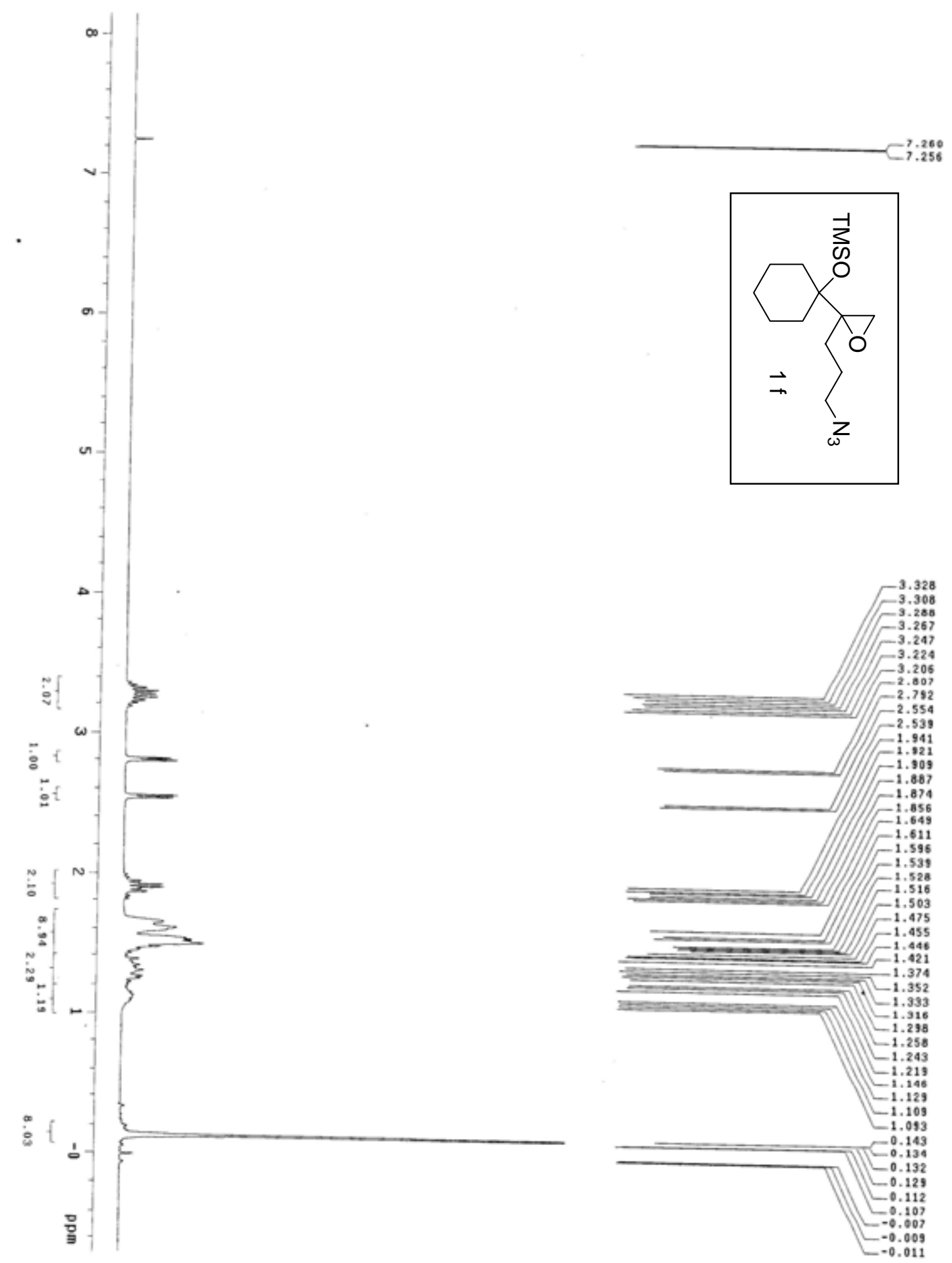
A Tandem Semipinacol/Schmidt Reaction Leading to Versatile and Efficient Approach to
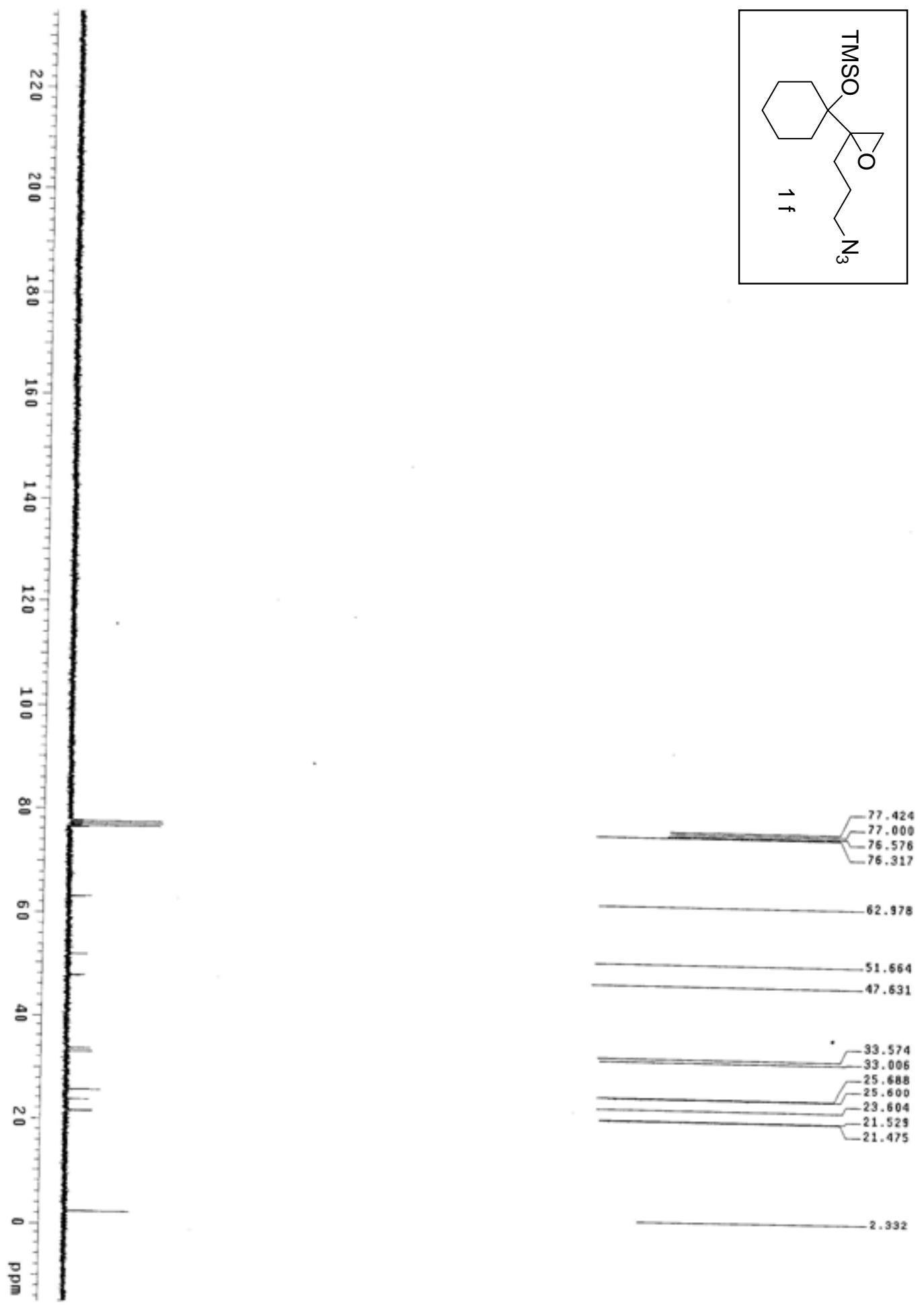
A Tandem Semipinacol/Schmidt Reaction Leading to Versatile and Efficient Approach to
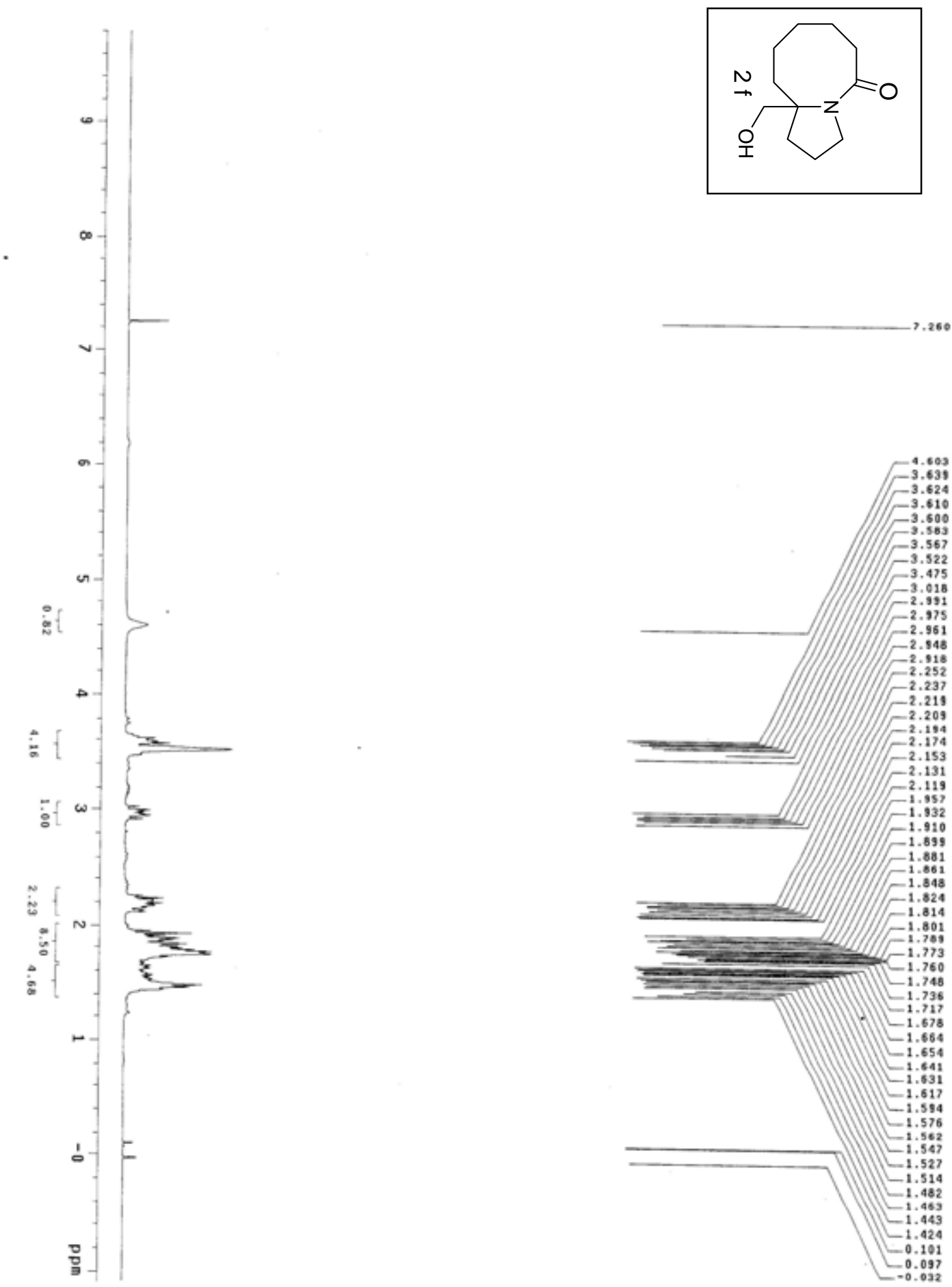
A Tandem Semipinacol/Schmidt Reaction Leading to Versatile and Efficient Approach to
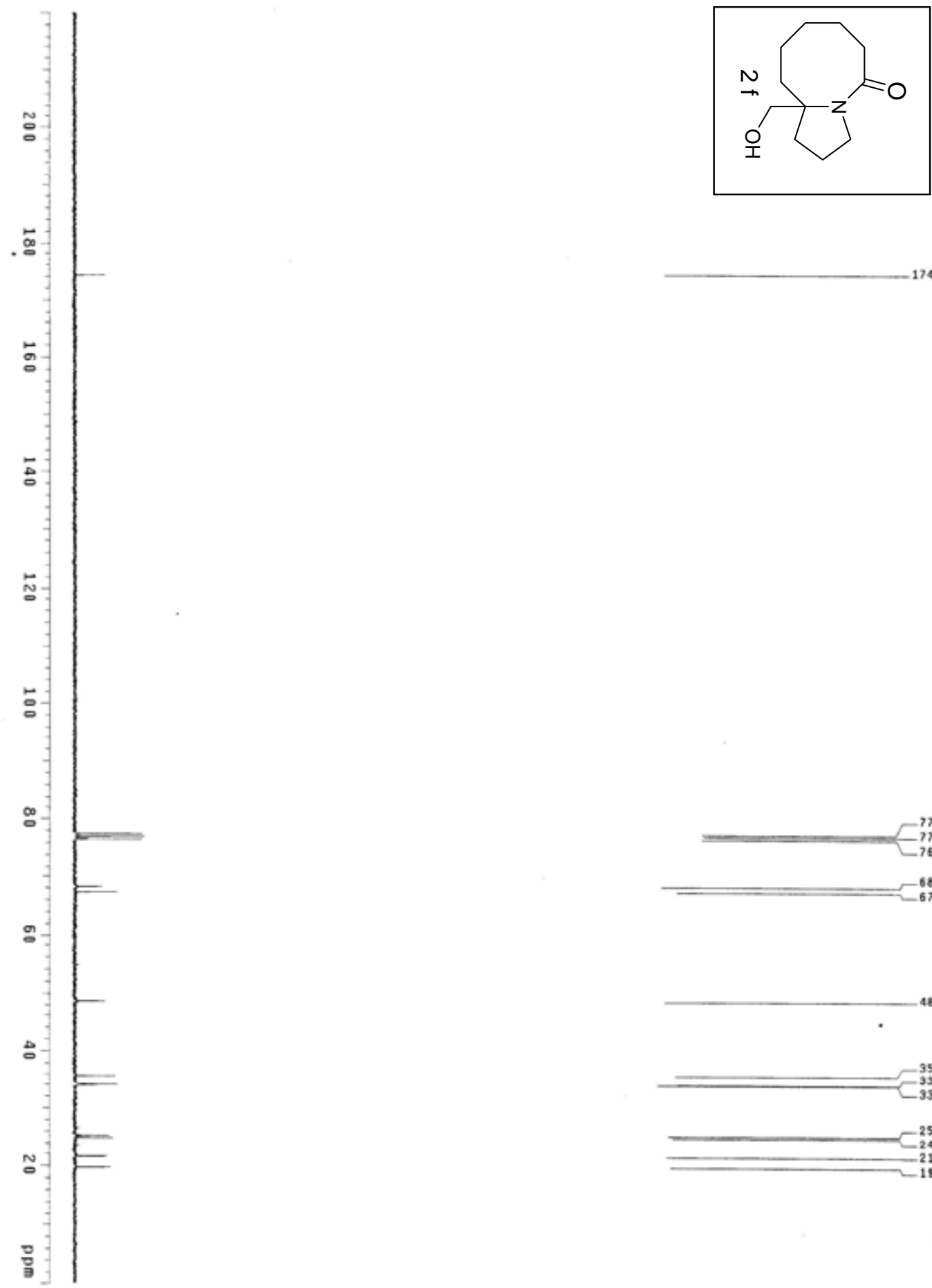

$-174.203$ 
A Tandem Semipinacol/Schmidt Reaction Leading to Versatile and Efficient Approach to
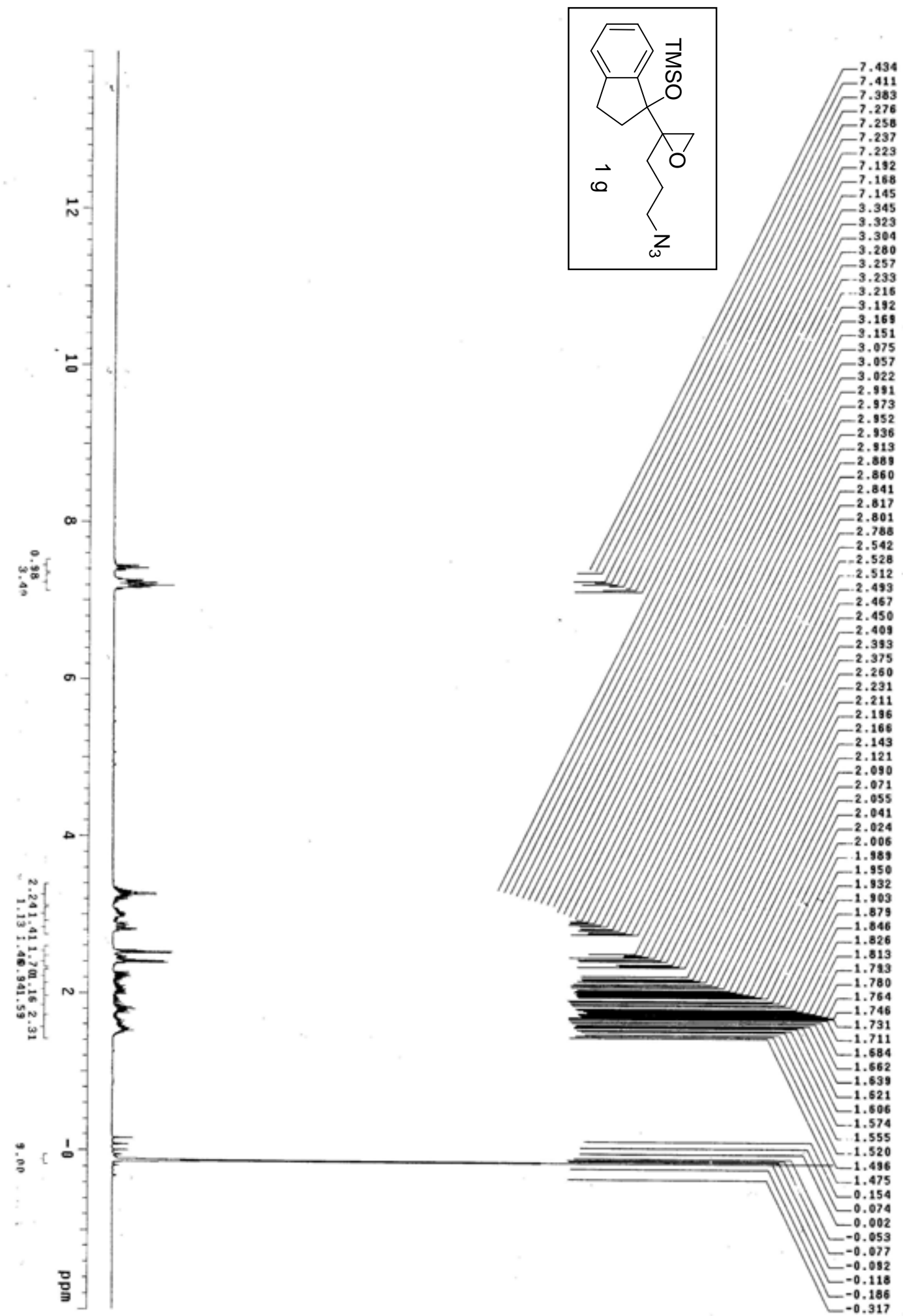
A Tandem Semipinacol/Schmidt Reaction Leading to Versatile and Efficient Approach to
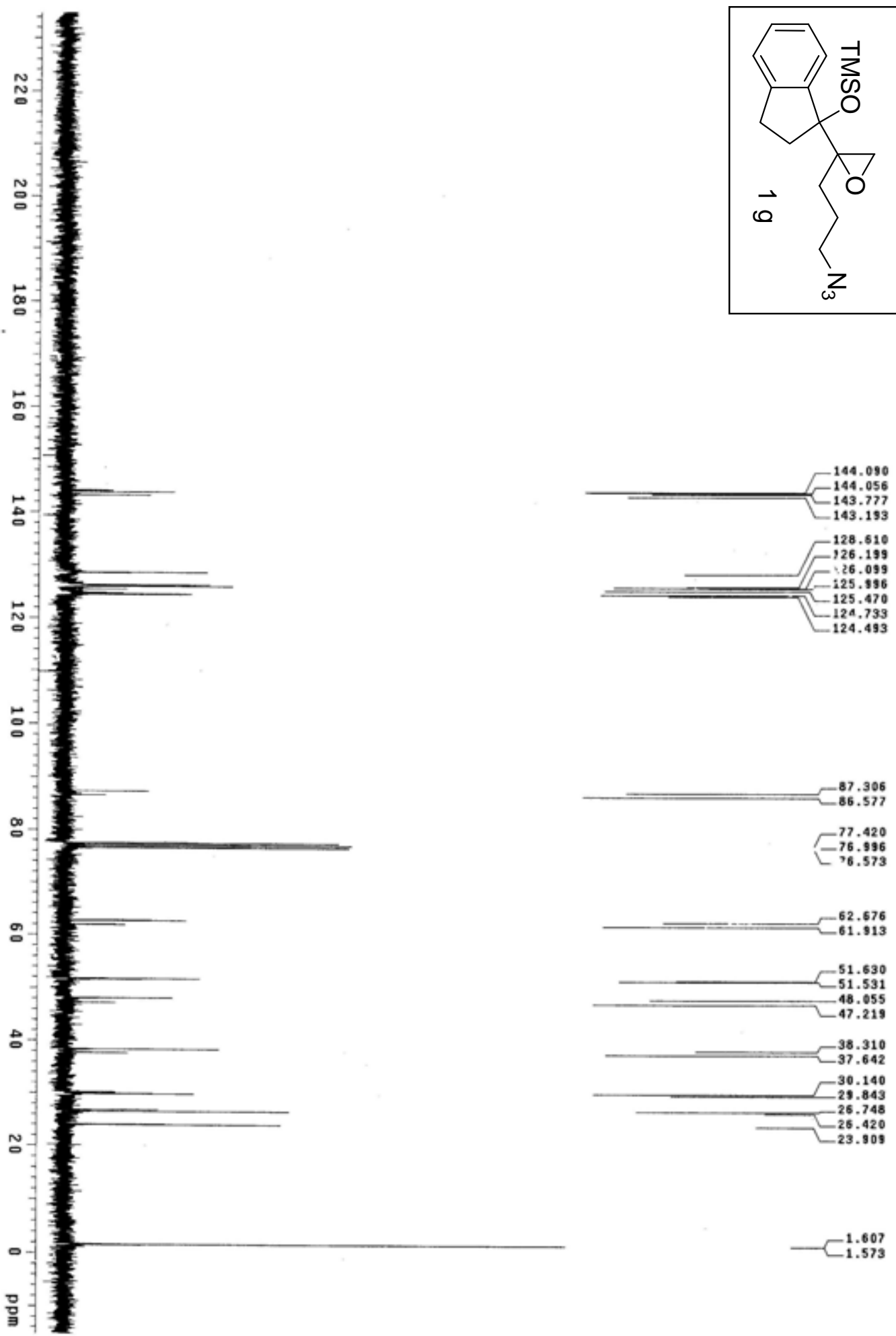
A Tandem Semipinacol/Schmidt Reaction Leading to Versatile and Efficient Approach to
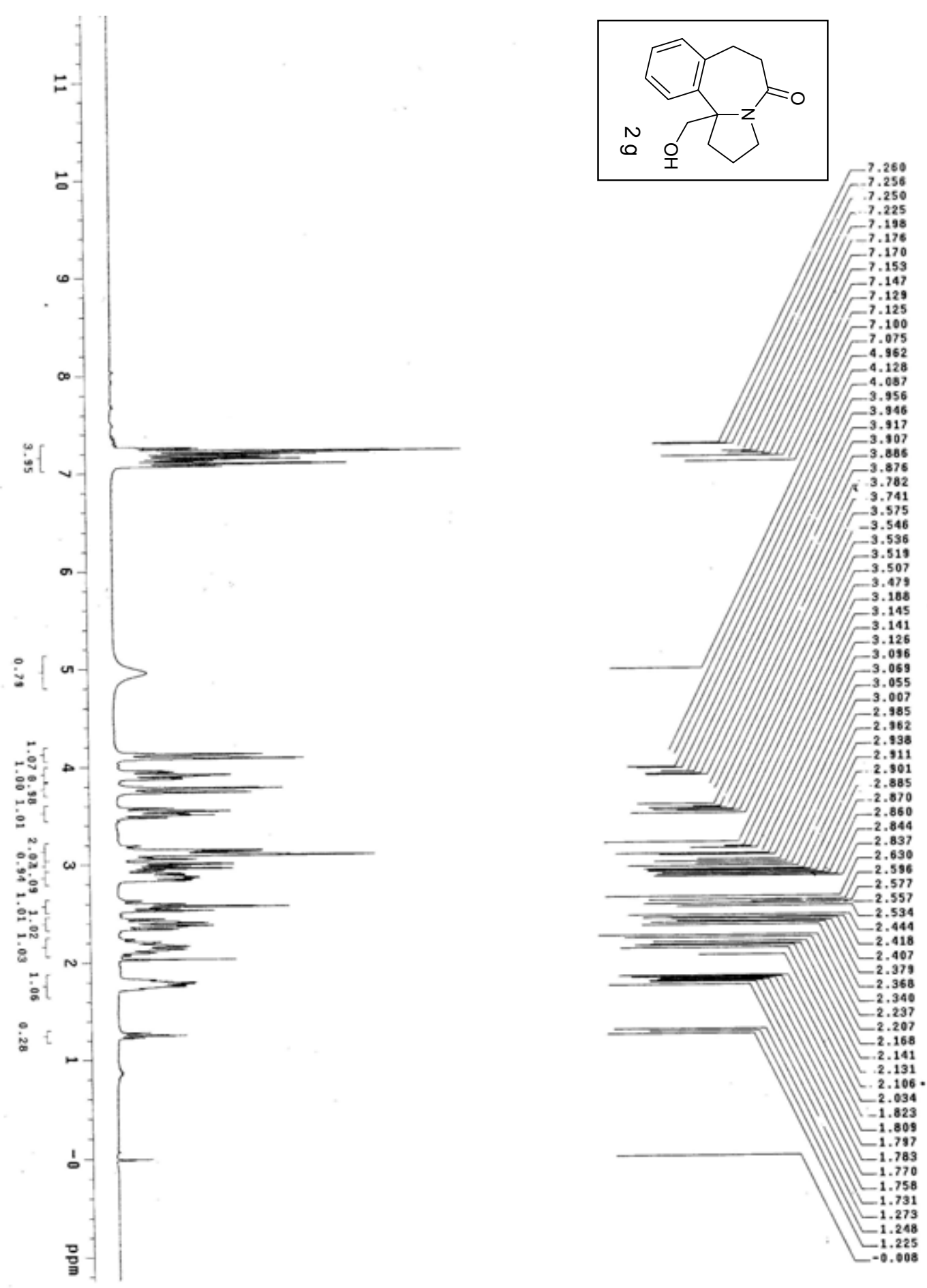
A Tandem Semipinacol/Schmidt Reaction Leading to Versatile and Efficient Approach to Azaquaternary Alkaloid Skeletons
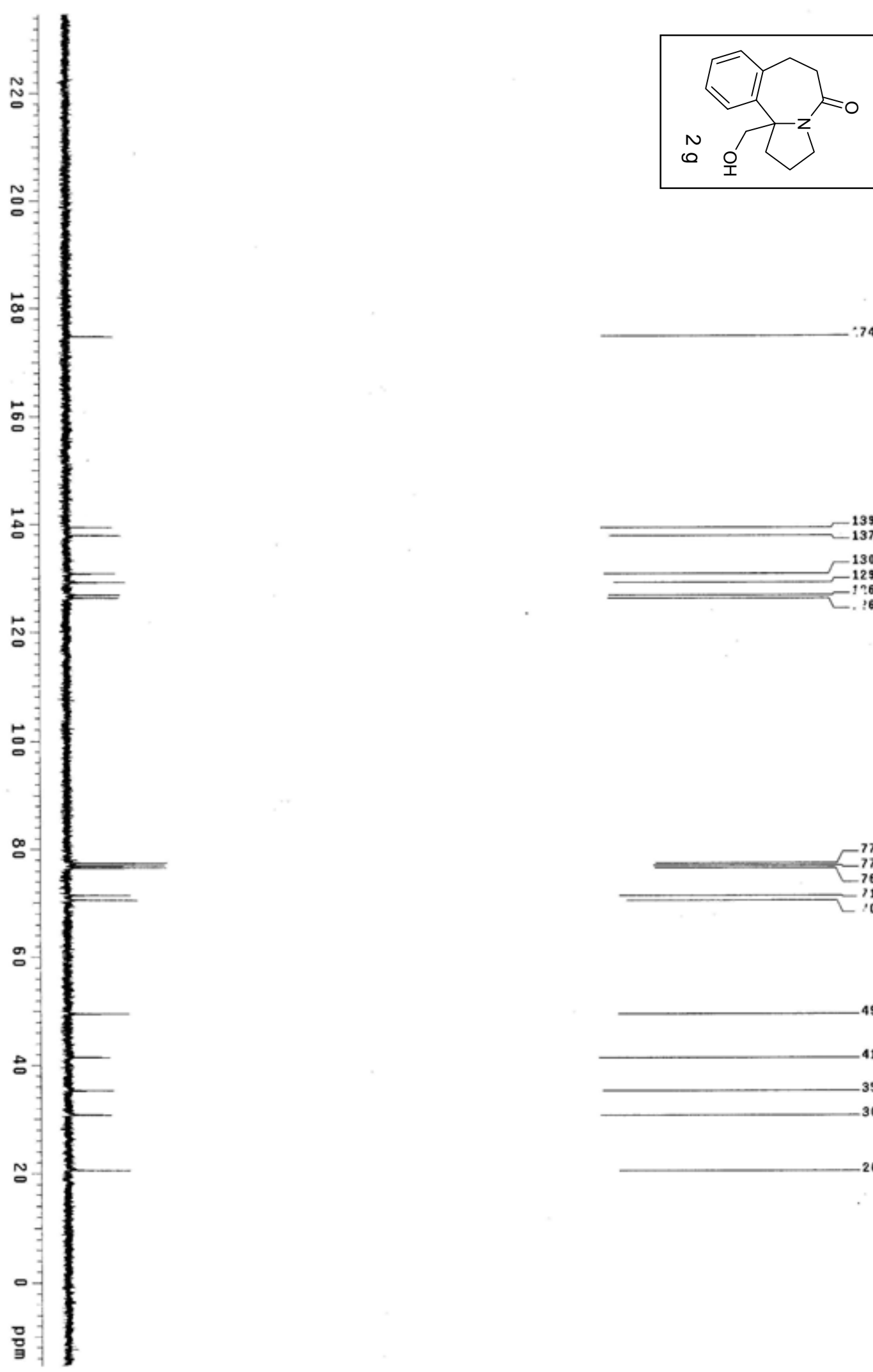

074.749
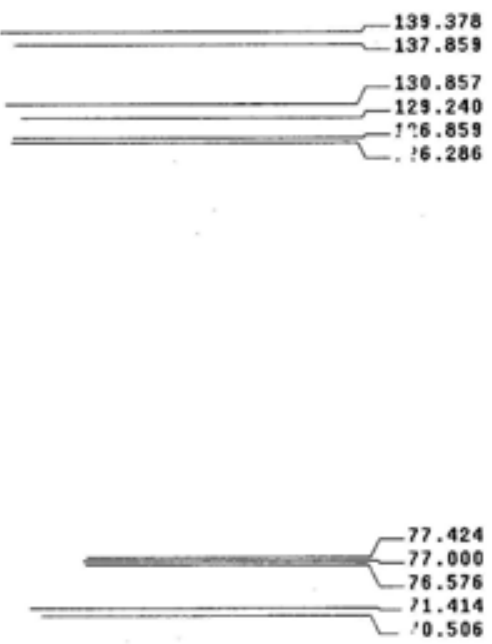

49.531

41.438

$-35.299$

$-30.759$

$-20.556$ 
A Tandem Semipinacol/Schmidt Reaction Leading to Versatile and Efficient Approach to
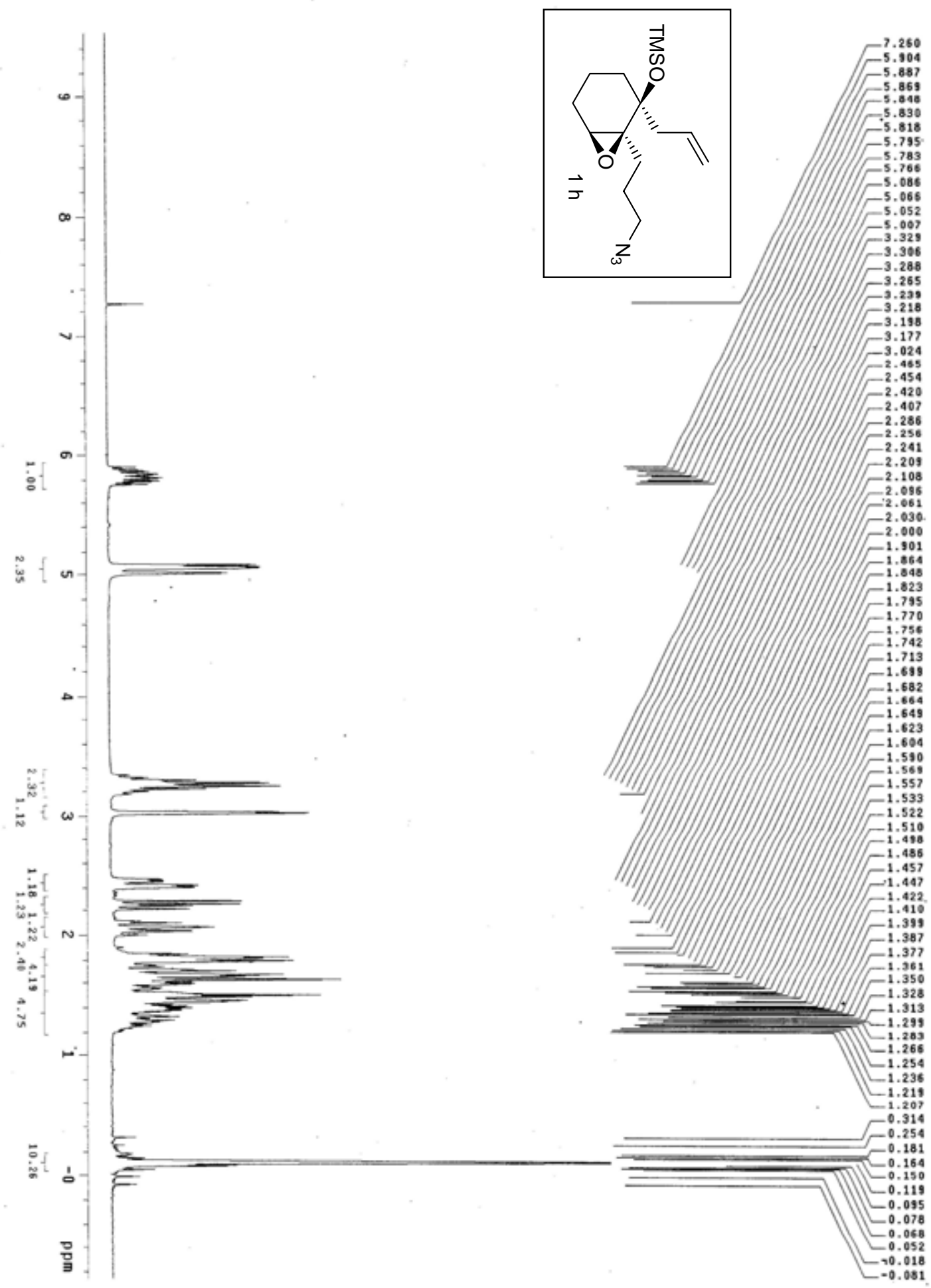
A Tandem Semipinacol/Schmidt Reaction Leading to Versatile and Efficient Approach to
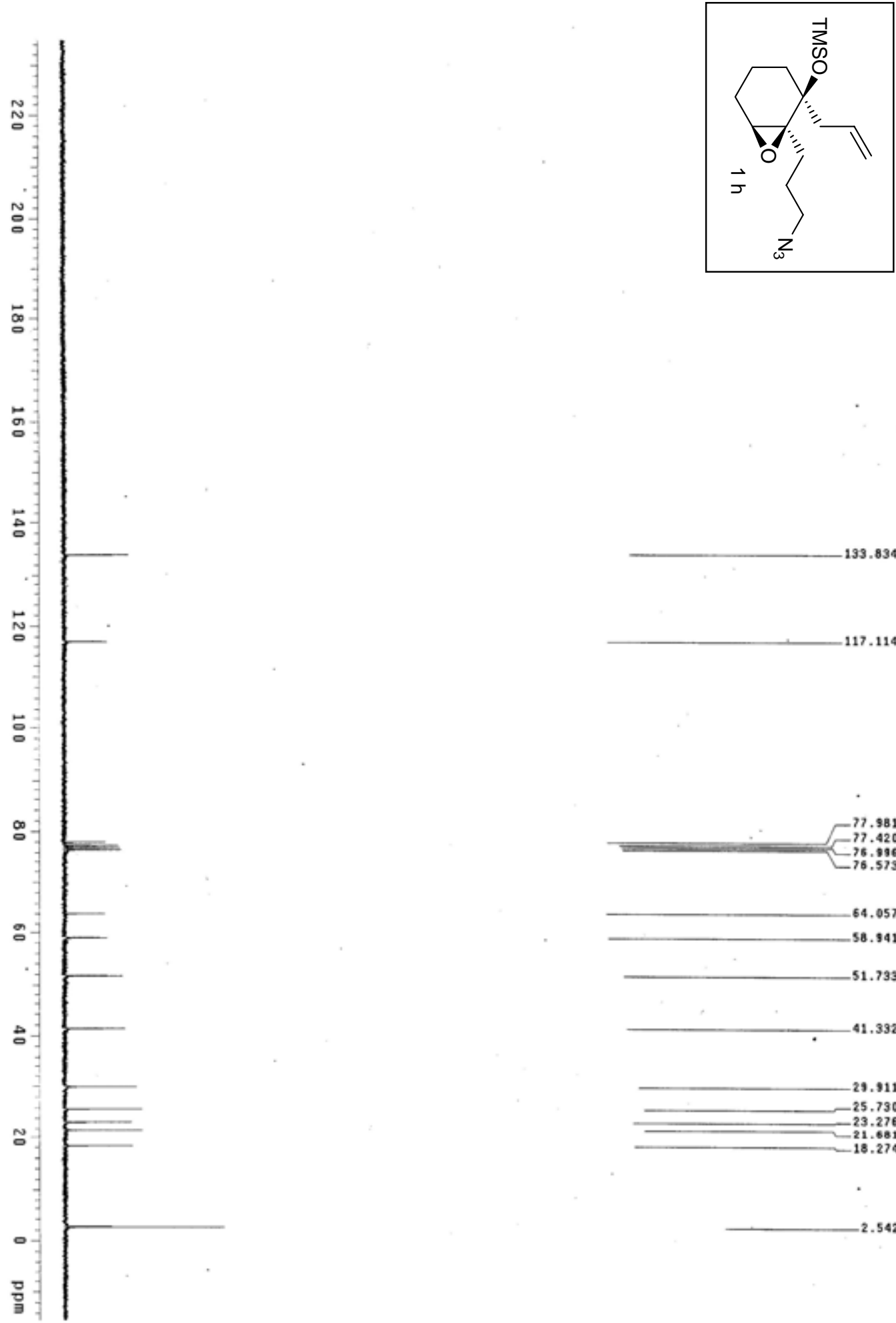

$-193.834$

117.114
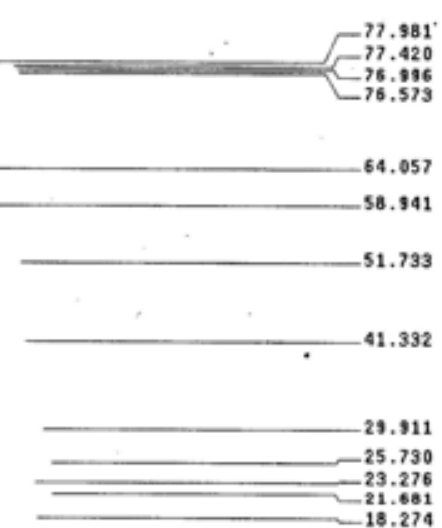

2.542 
A Tandem Semipinacol/Schmidt Reaction Leading to Versatile and Efficient Approach to
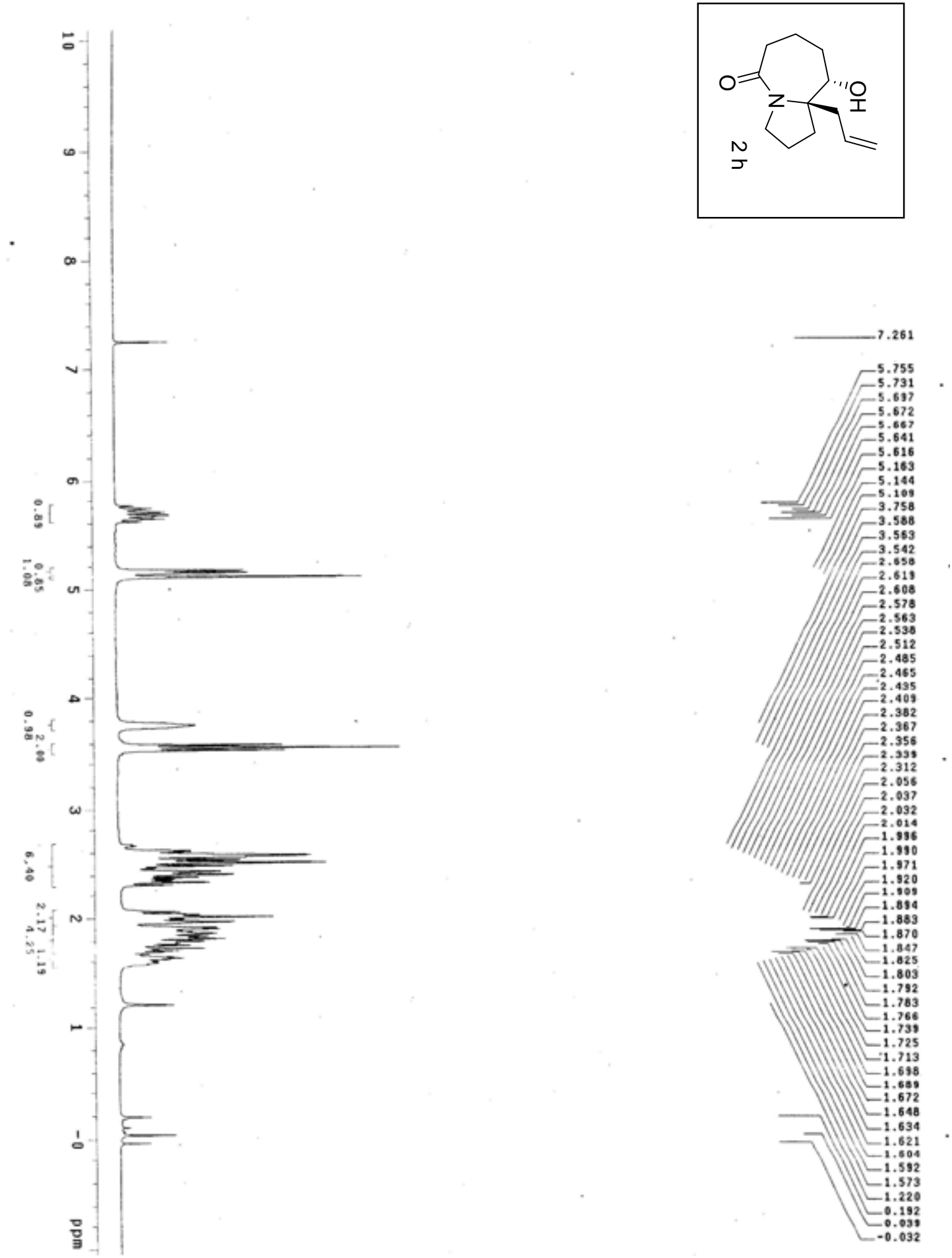
A Tandem Semipinacol/Schmidt Reaction Leading to Versatile and Efficient Approach to
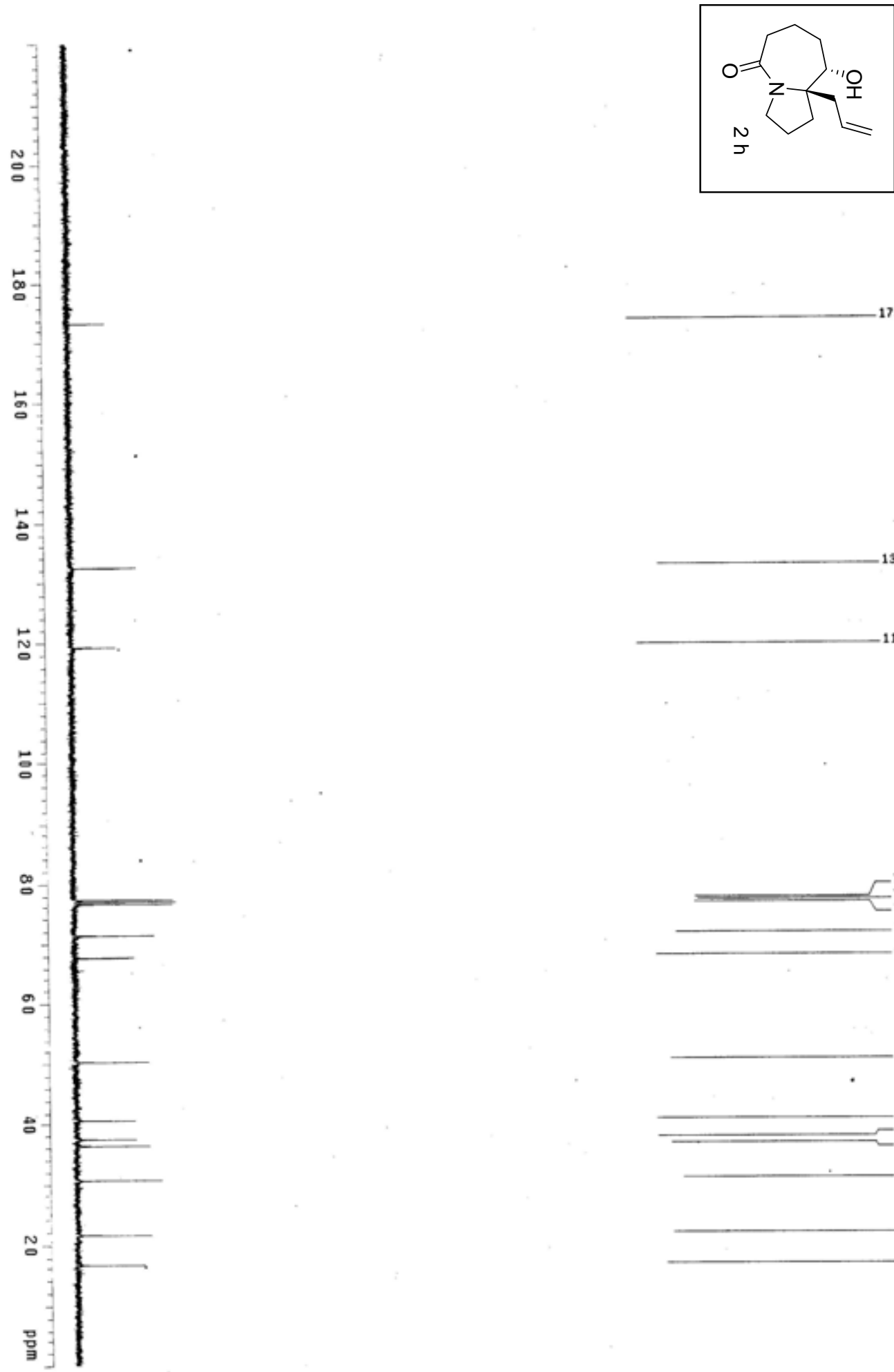

73. 451

$-132.346$

$-119.262$
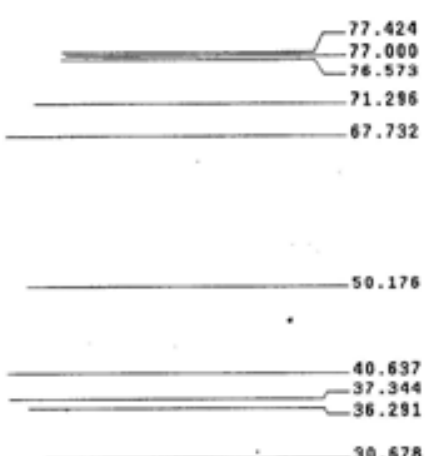

$-21.532$

$-16.591$ 
A Tandem Semipinacol/Schmidt Reaction Leading to Versatile and Efficient Approach to
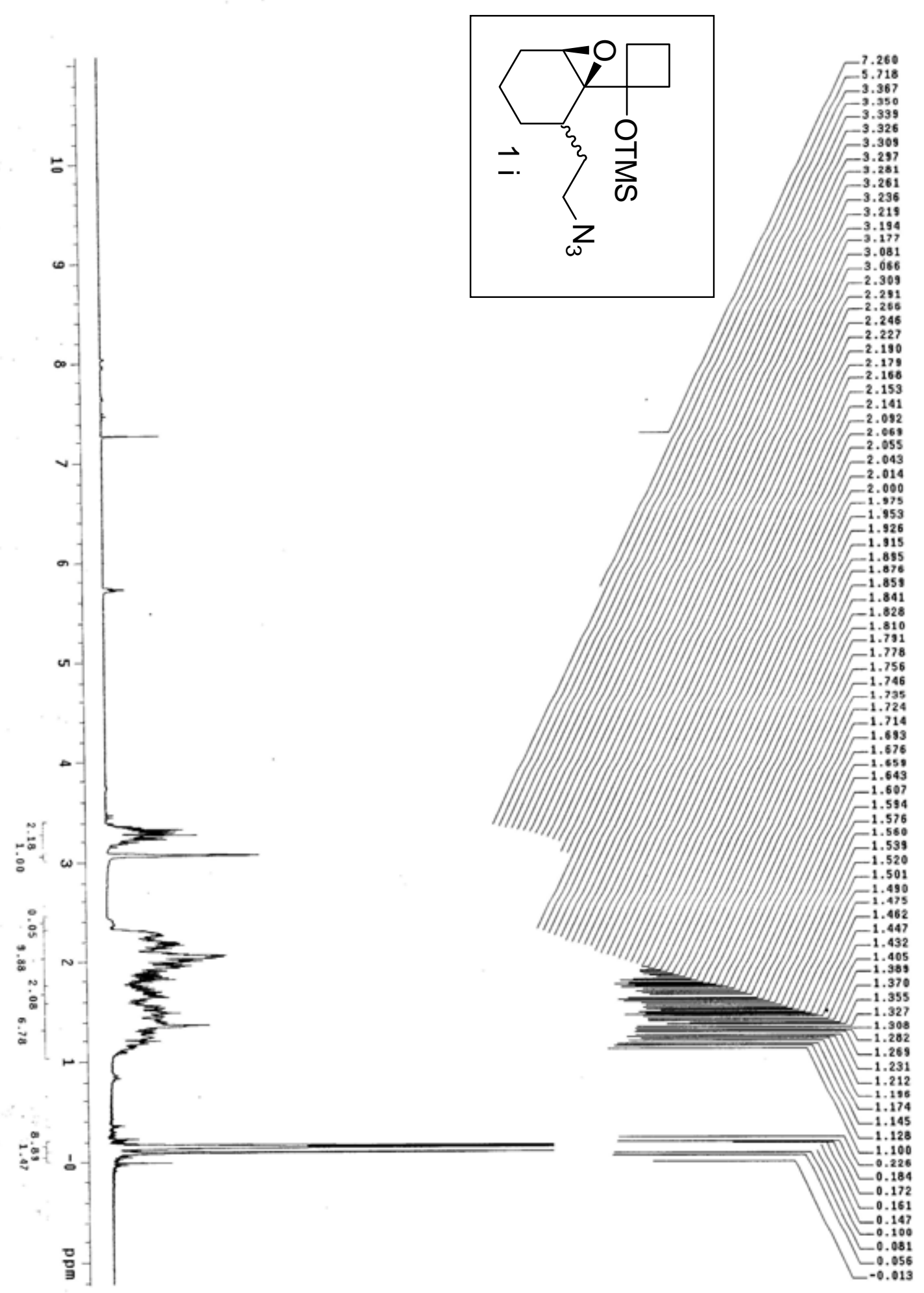
A Tandem Semipinacol/Schmidt Reaction Leading to Versatile and Efficient Approach to
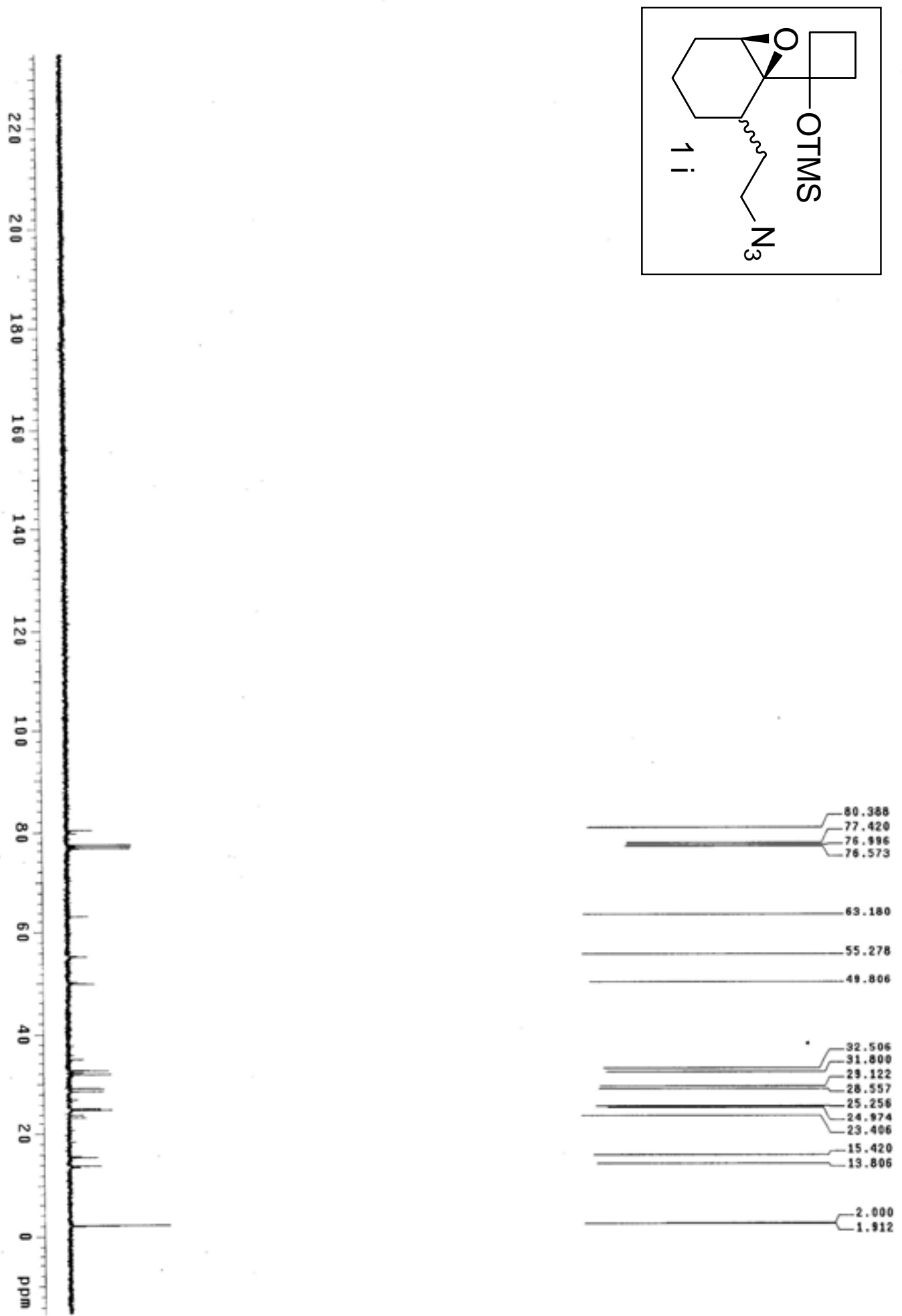
A Tandem Semipinacol/Schmidt Reaction Leading to Versatile and Efficient Approach to
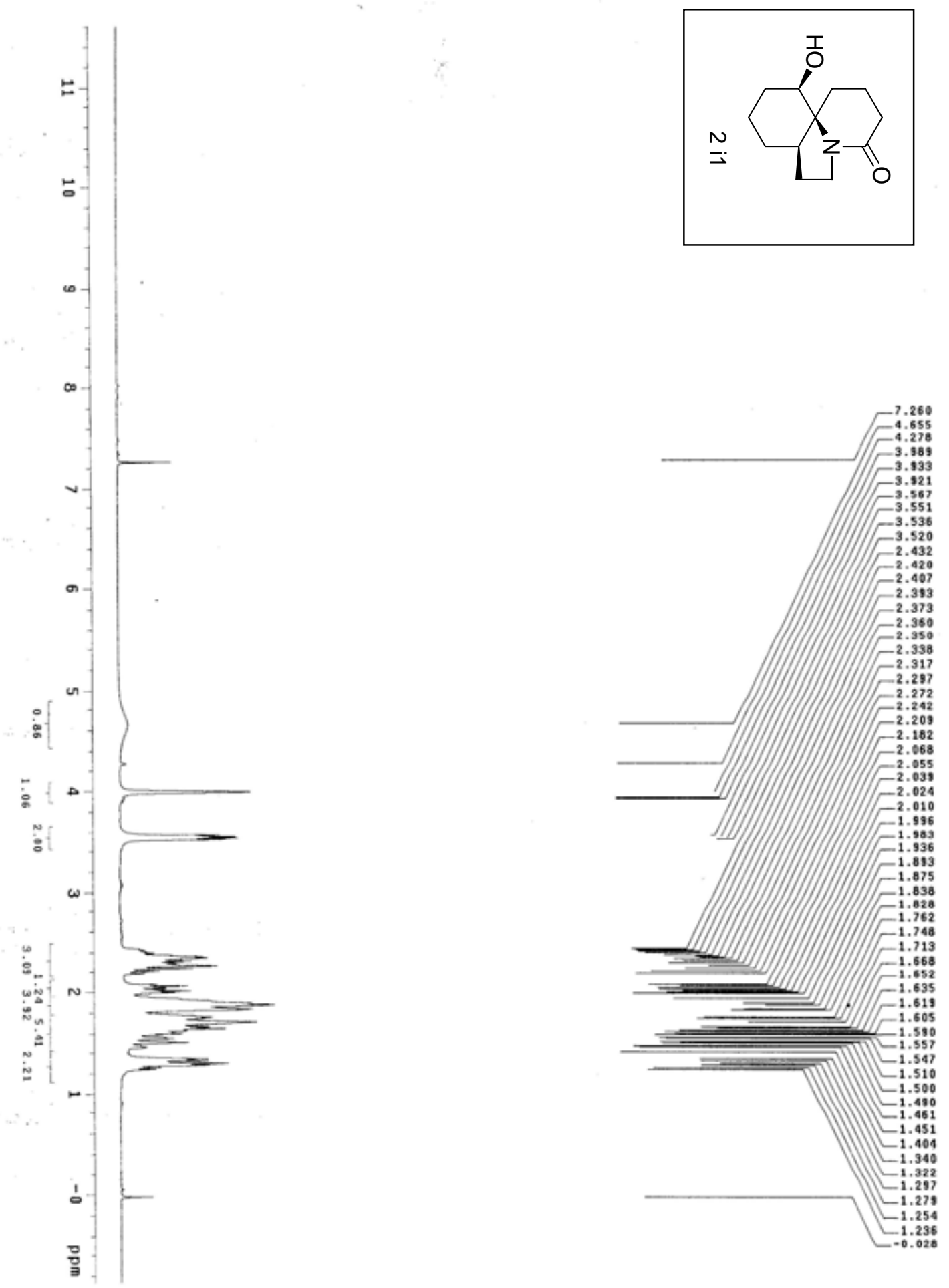
A Tandem Semipinacol/Schmidt Reaction Leading to Versatile and Efficient Approach to
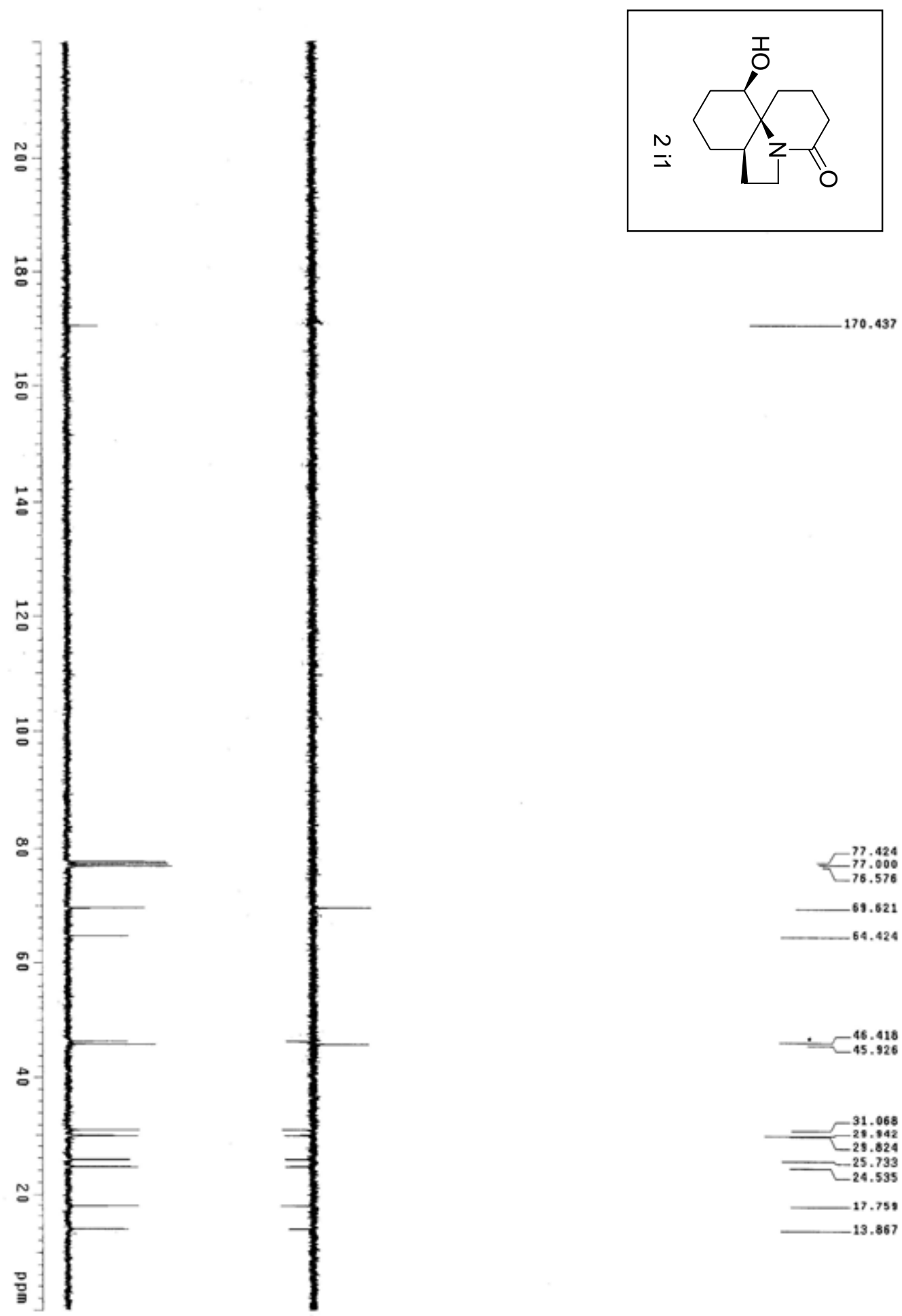
A Tandem Semipinacol/Schmidt Reaction Leading to Versatile and Efficient Approach to
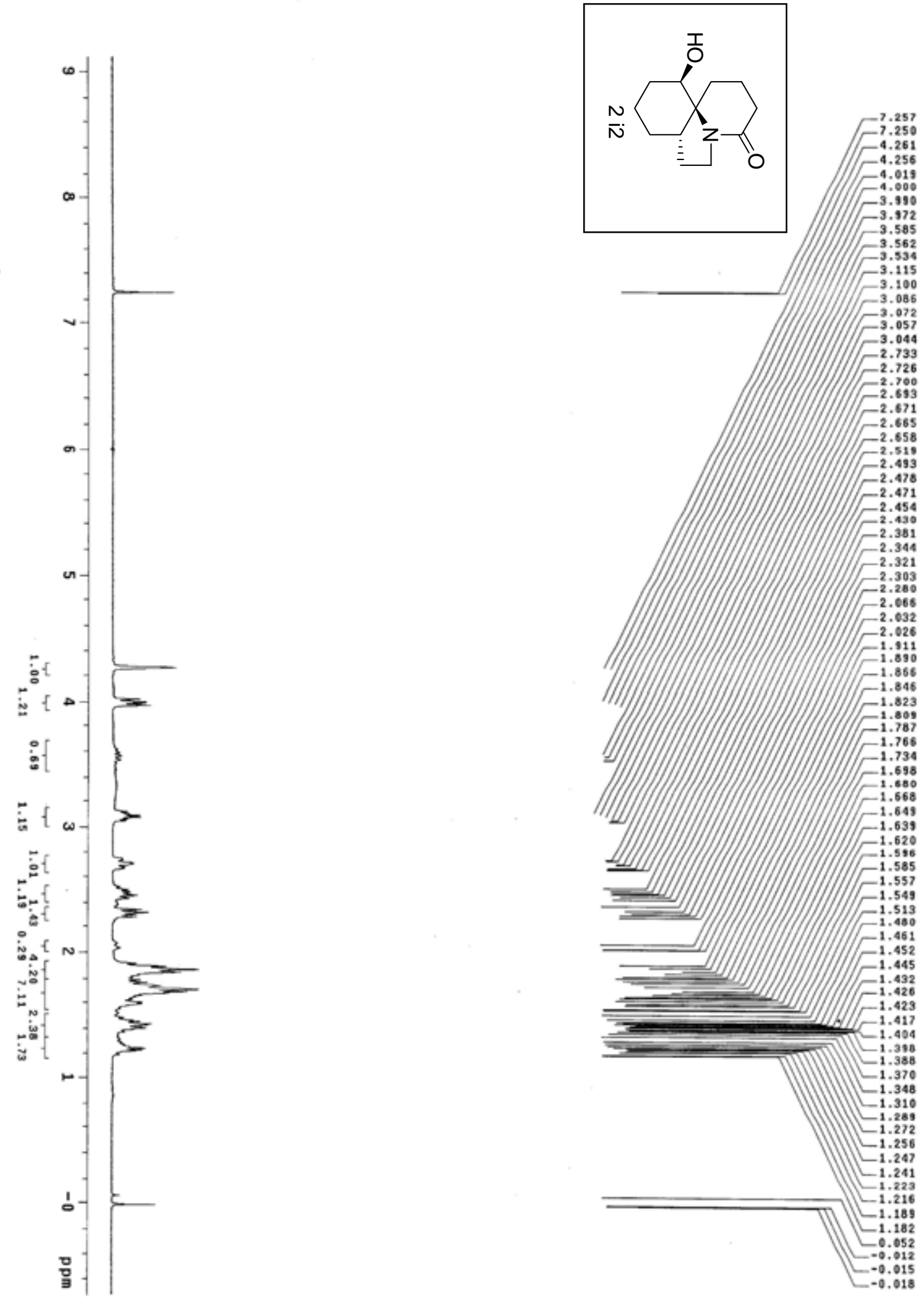
A Tandem Semipinacol/Schmidt Reaction Leading to Versatile and Efficient Approach to
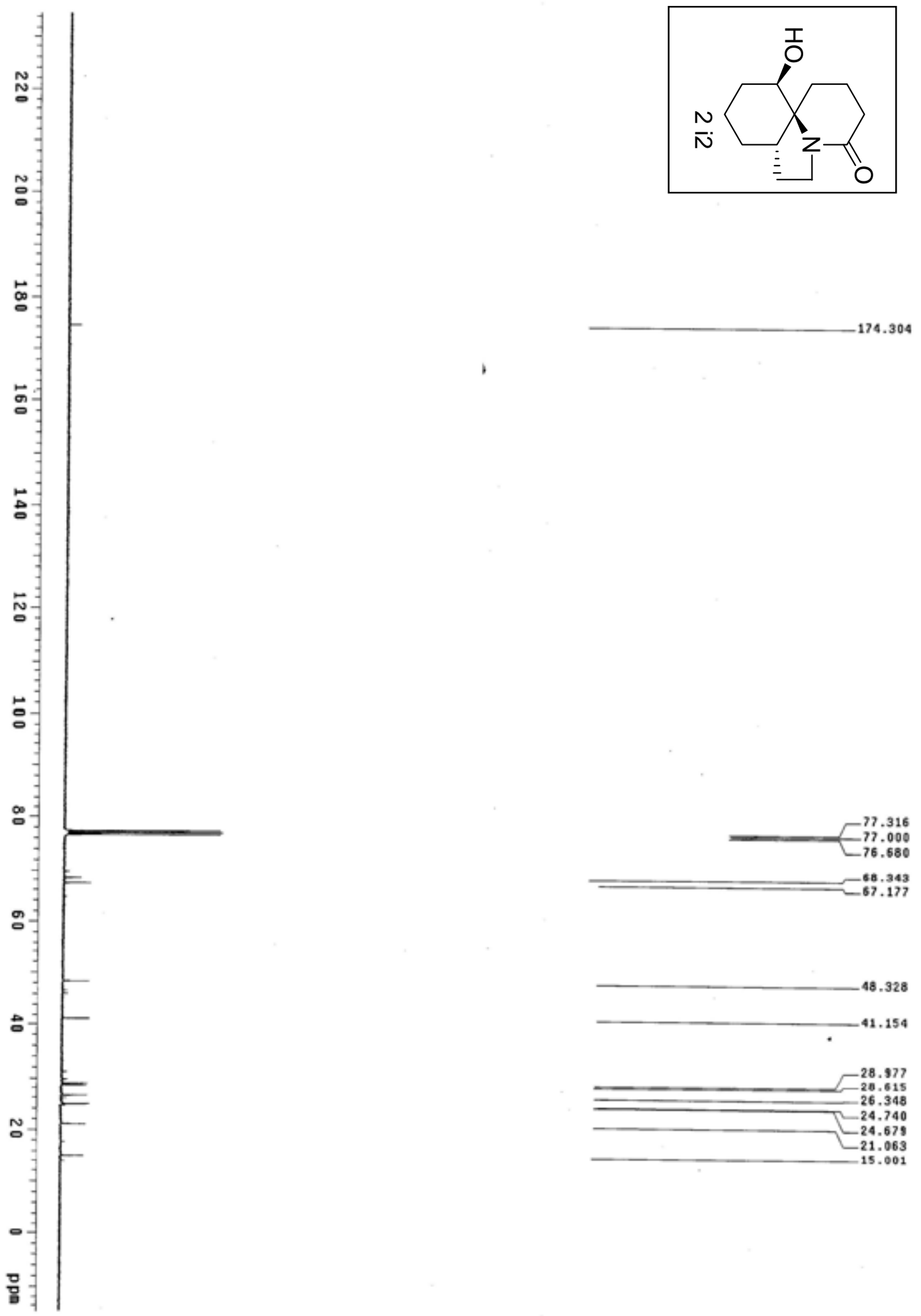
A Tandem Semipinacol/Schmidt Reaction Leading to Versatile and Efficient Approach to

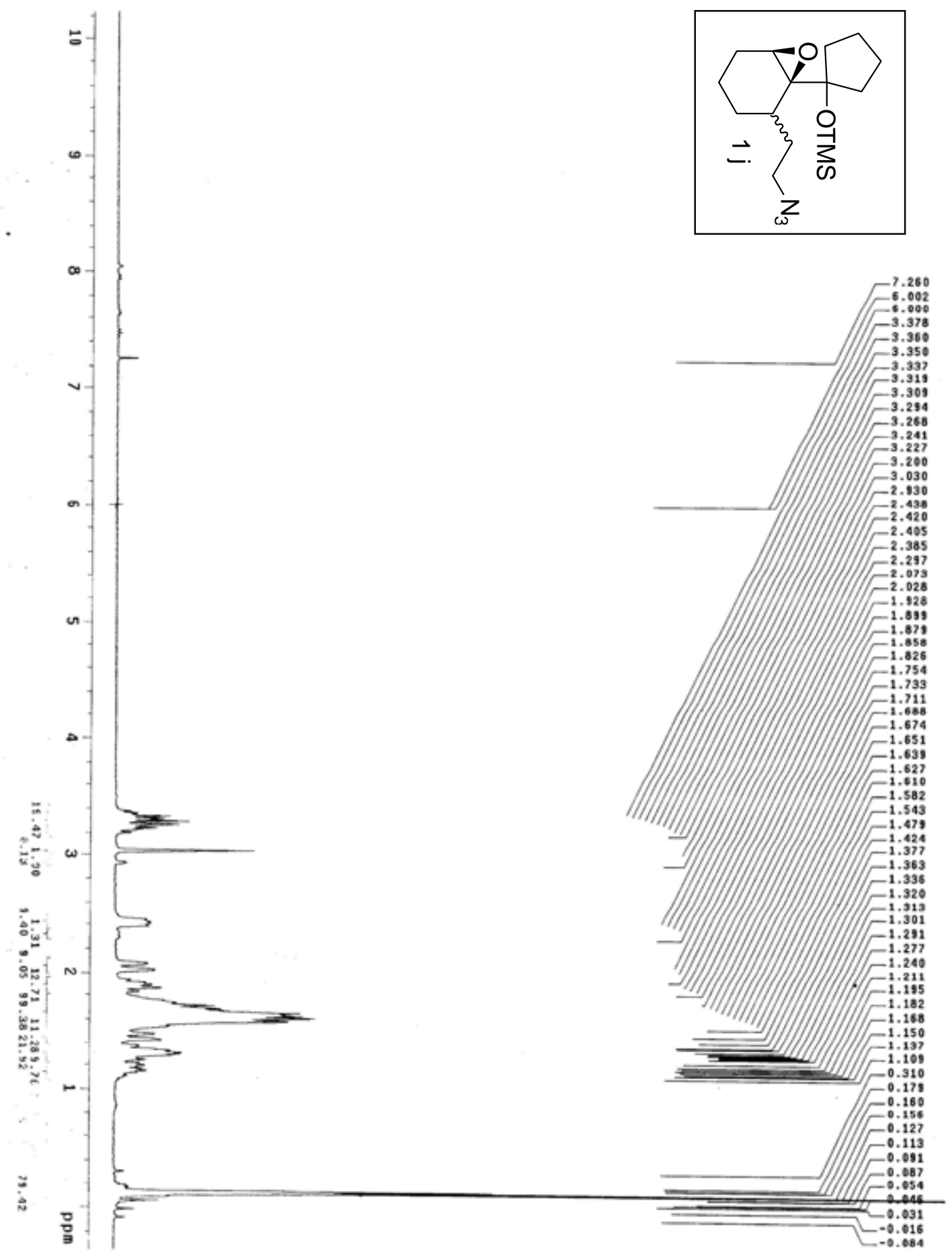


A Tandem Semipinacol/Schmidt Reaction Leading to Versatile and Efficient Approach to
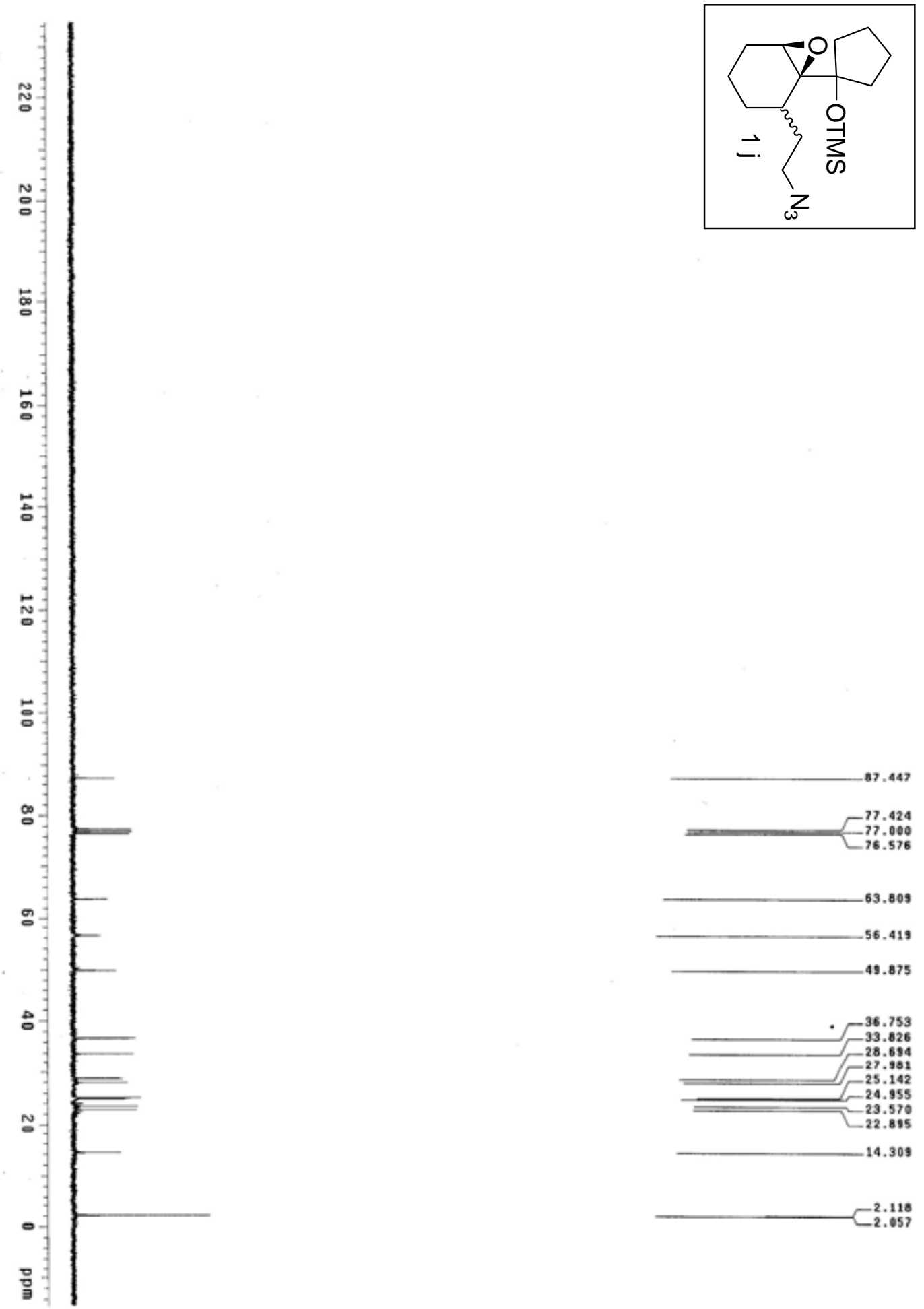

$-14.309$

$\left\ulcorner_{2.057}^{2.118}\right.$ 
A Tandem Semipinacol/Schmidt Reaction Leading to Versatile and Efficient Approach to
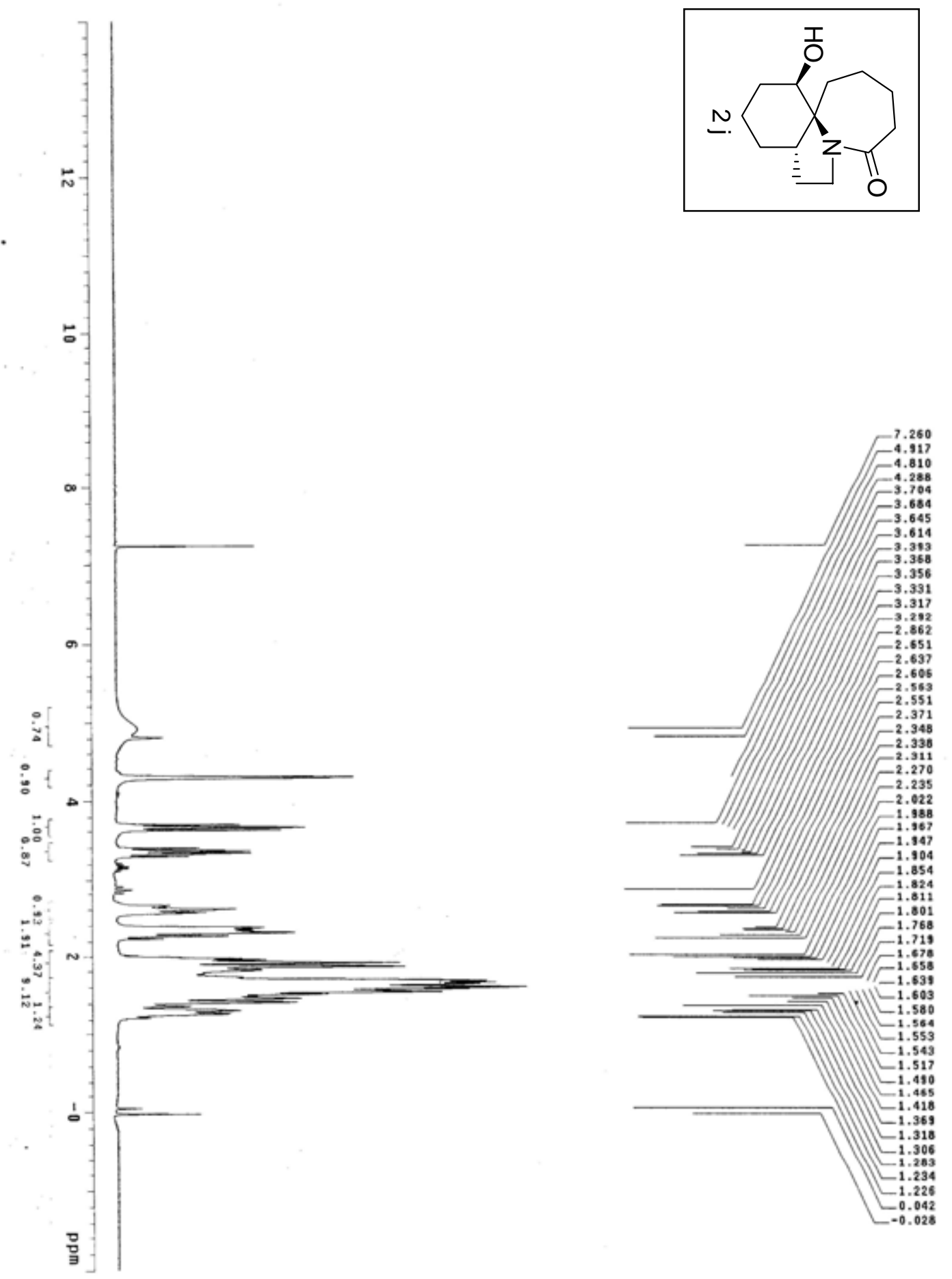
A Tandem Semipinacol/Schmidt Reaction Leading to Versatile and Efficient Approach to
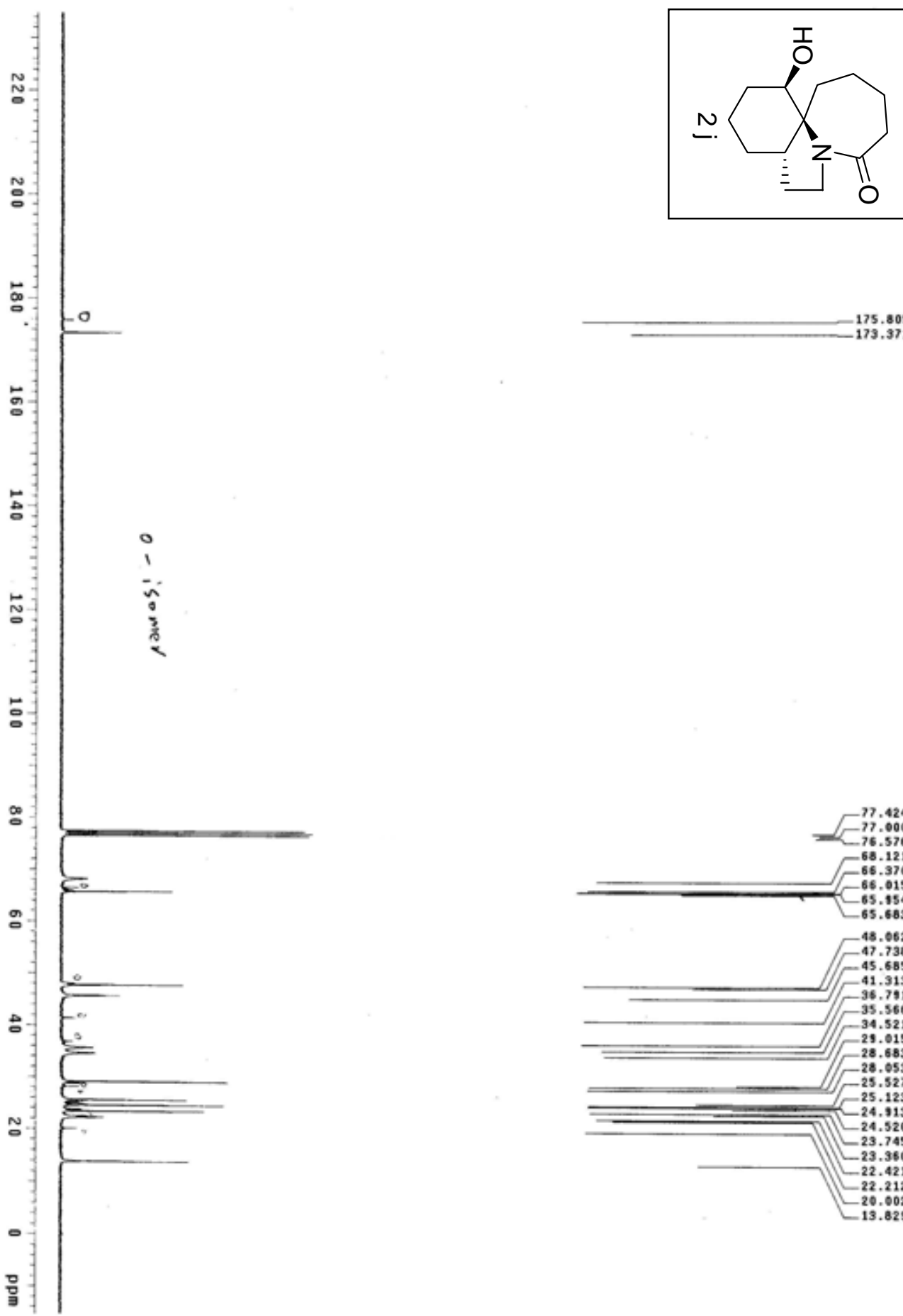

175.805

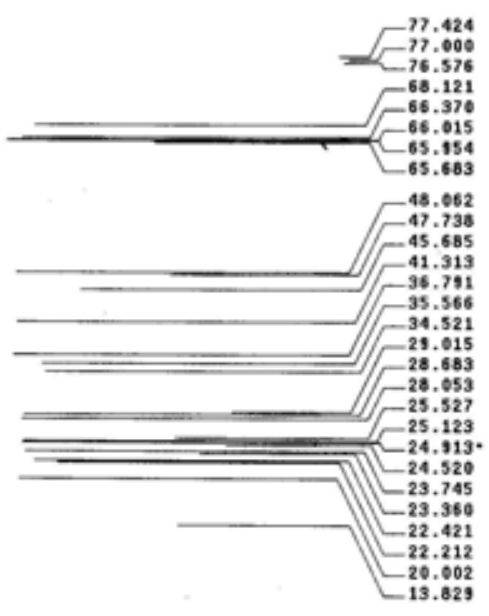


A Tandem Semipinacol/Schmidt Reaction Leading to Versatile and Efficient Approach to
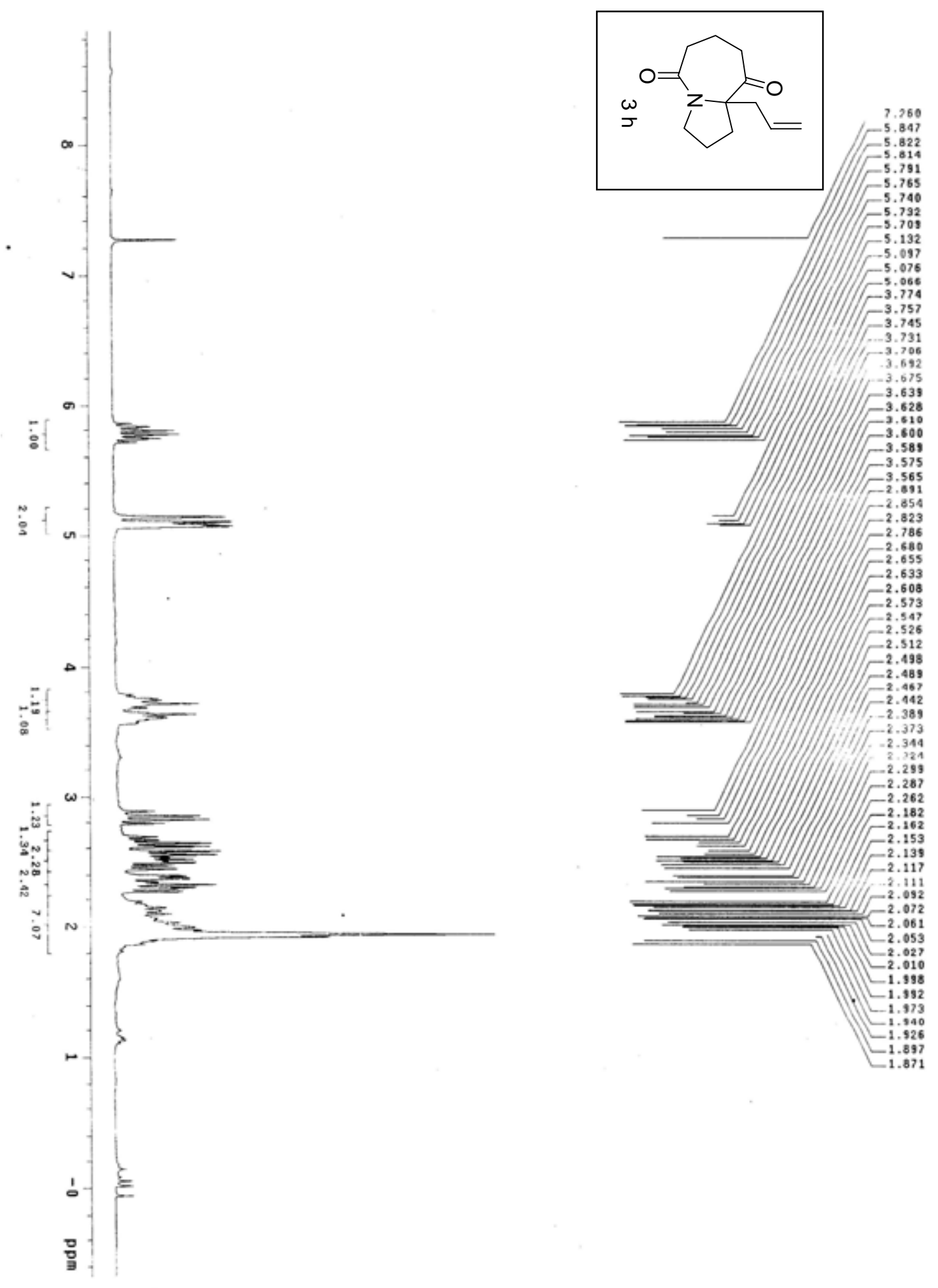
A Tandem Semipinacol/Schmidt Reaction Leading to Versatile and Efficient Approach to
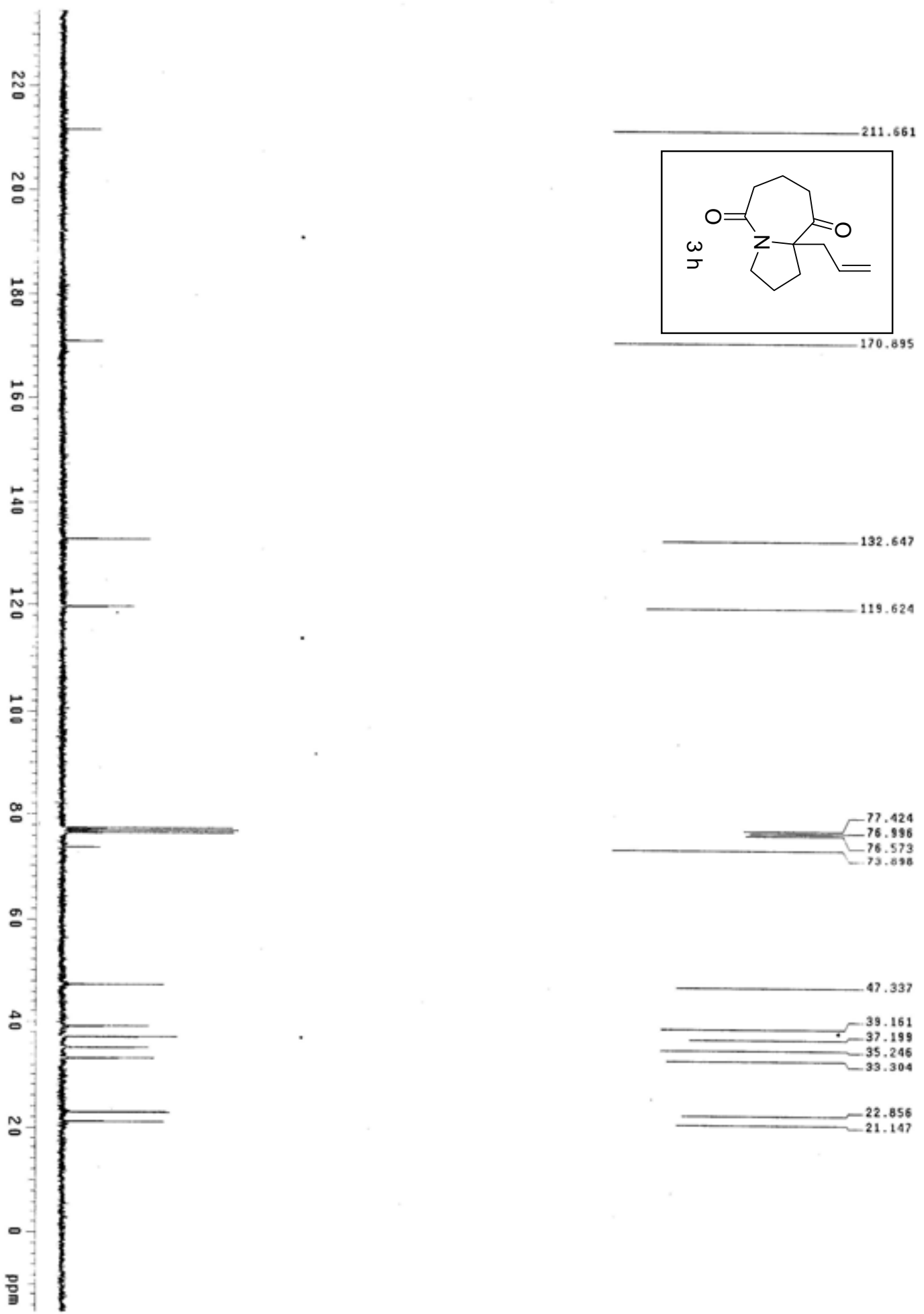

132.647

$-119.624$

$-77.424$

$-76.573$

33.690

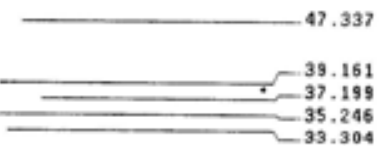

22.856

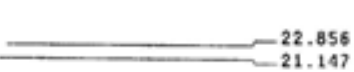


A Tandem Semipinacol/Schmidt Reaction Leading to Versatile and Efficient Approach to

850.0

608.0
$\angle 98.0$

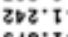

528.
$606 \cdot 1$

$006 \cdot 1=$

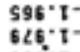

$686^{\circ} \mathrm{i}$

$800 \cdot 2$
$920 \cdot \frac{2}{2}$

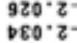

$650 \cdot 2$

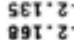

$\begin{array}{lll}89 \mathrm{r} \cdot & z- \\ 002 \cdot z- & -1\end{array}$

$802 \cdot z-10$

$898 \cdot z-1$

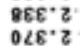

$668^{\circ} 8700$

$196 \cdot 3-$

To5. 2

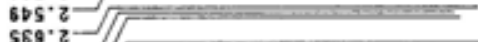

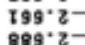

โระ. 2

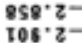

$526 \cdot 2-$

$056^{\circ} 2$

$2 z 9 \cdot \varepsilon-1$
obg. $8-1$

SL9. $8-$

$202 \cdot \varepsilon-$

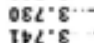

$092^{\circ}$

$\mid+5$

$\sqrt{\sqrt{1}}$

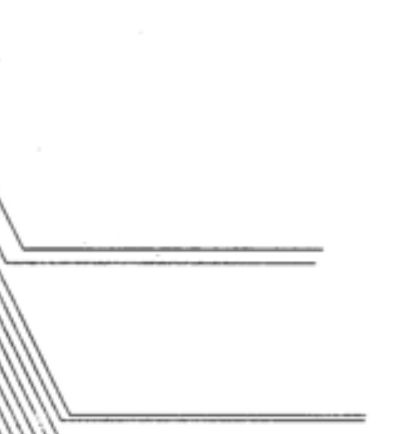

ivi
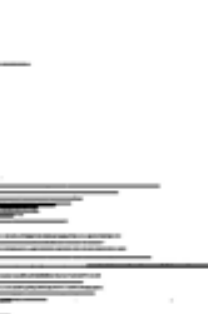

I $2 L^{\circ}$
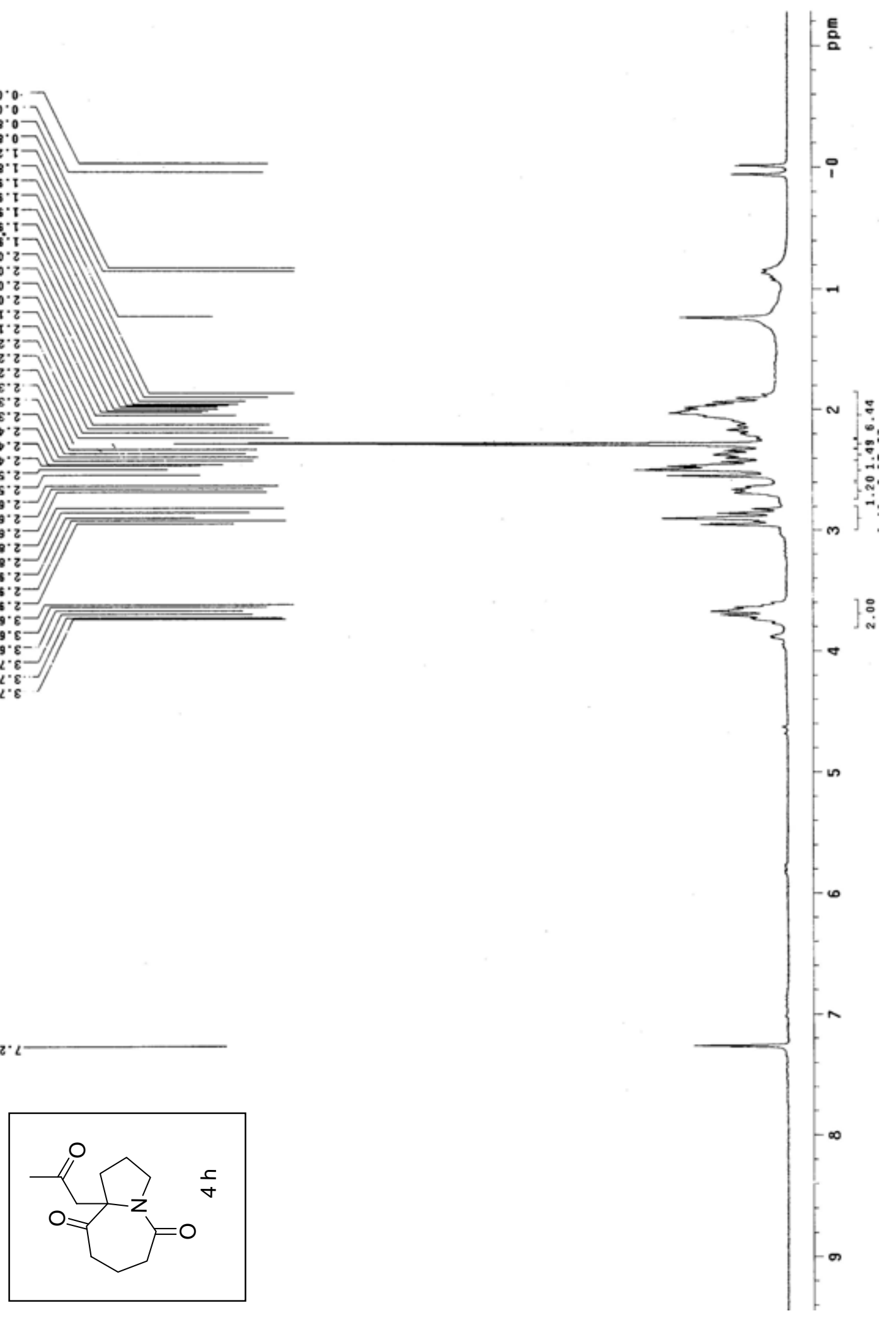

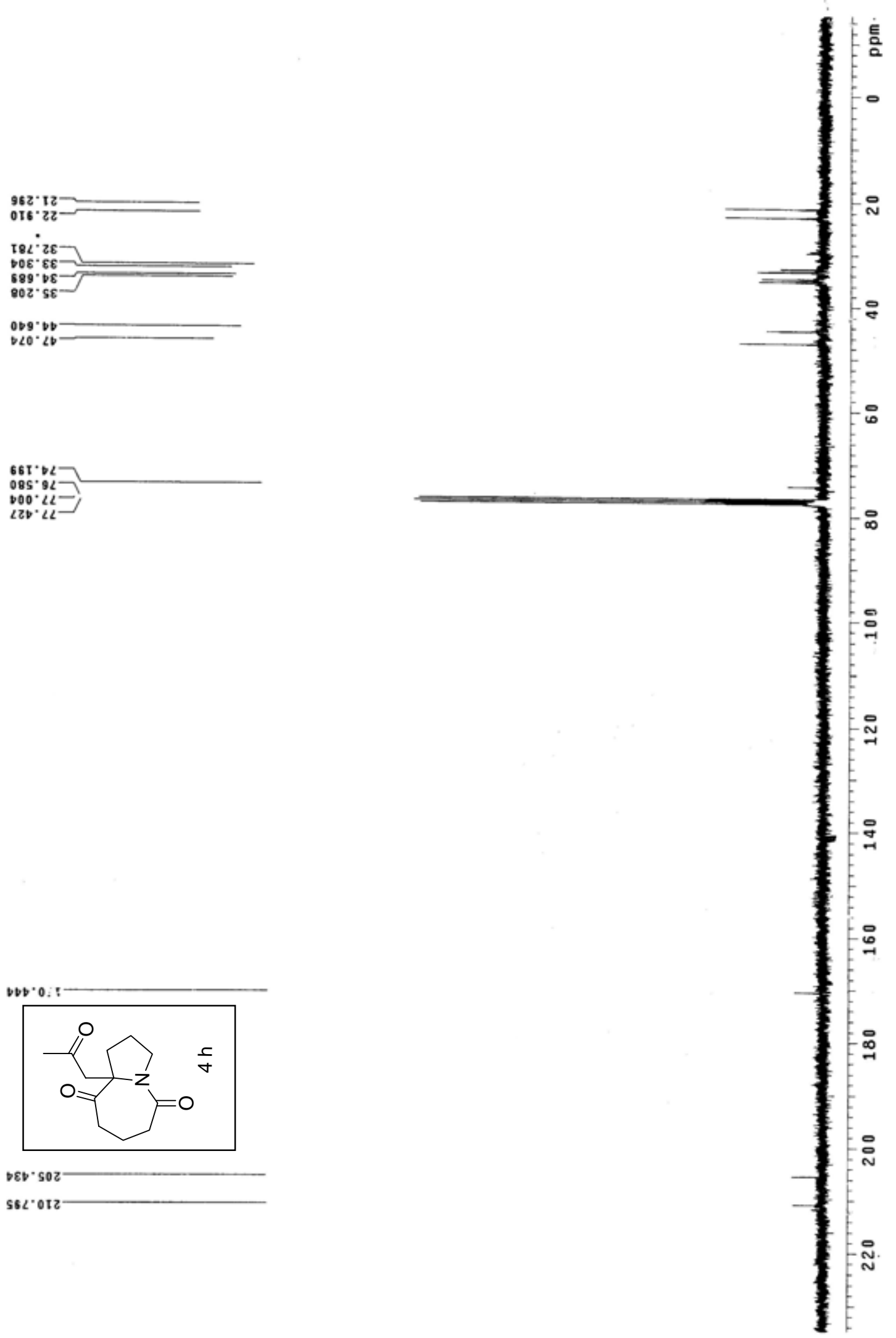

$D 8 b^{\circ} \mathrm{soz}$

SSL:0t2

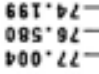

$000 \% \angle L$

$\angle z b^{\circ} \cdot 2 l-$ 
A Tandem Semipinacol/Schmidt Reaction Leading to Versatile and Efficient Approach to
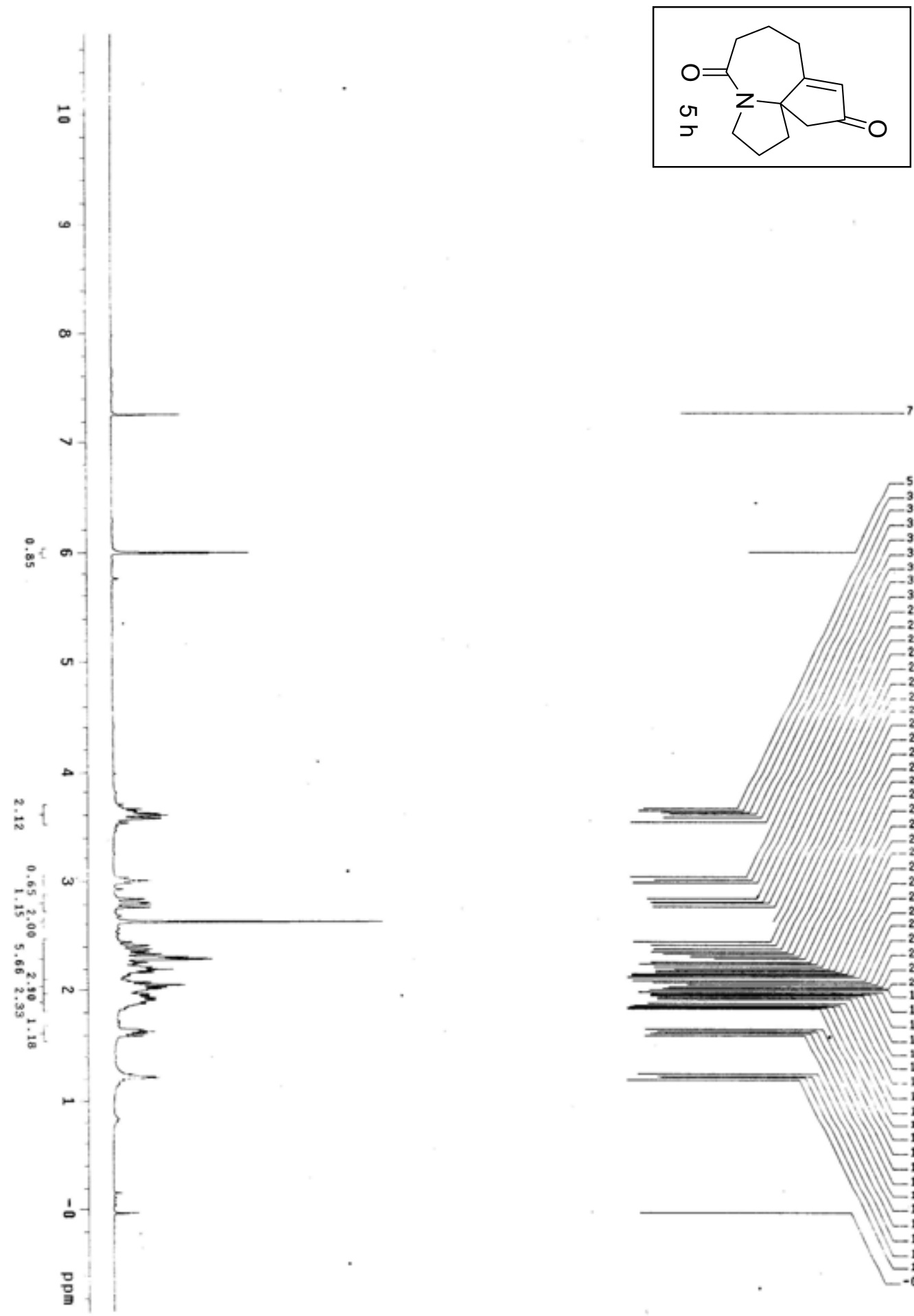

$-7.260$

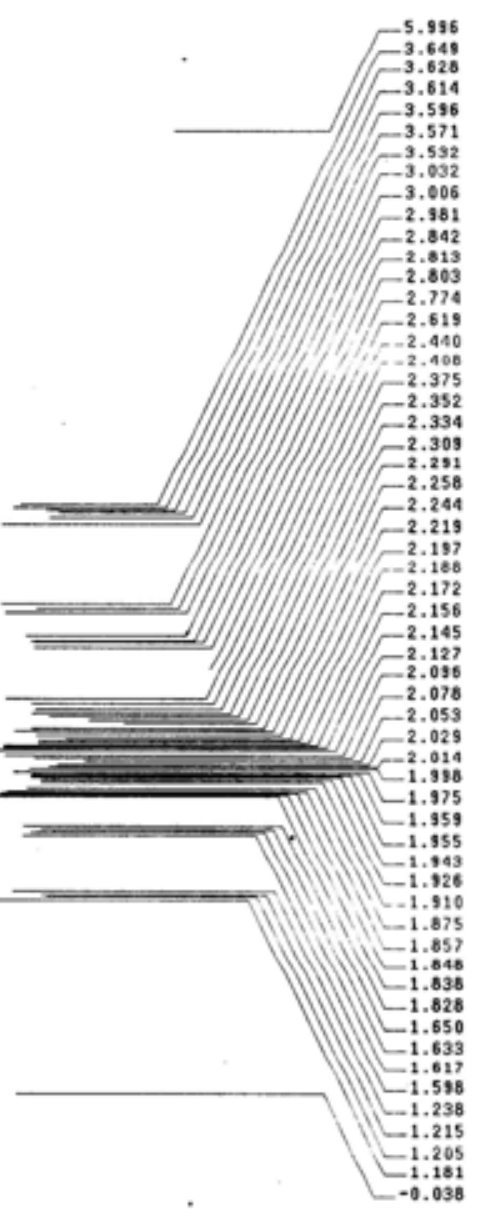


A Tandem Semipinacol/Schmidt Reaction Leading to Versatile and Efficient Approach to
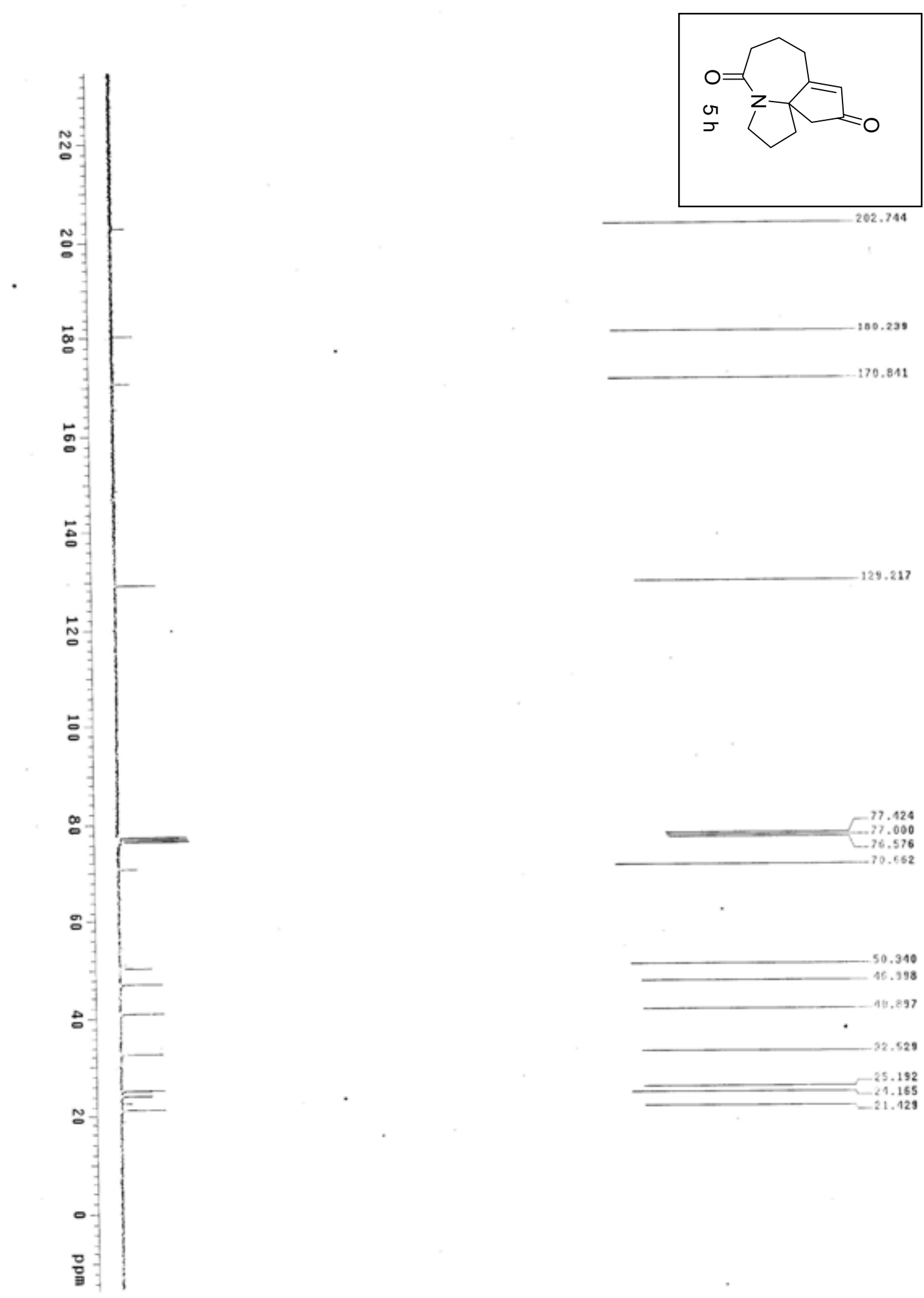

129.217

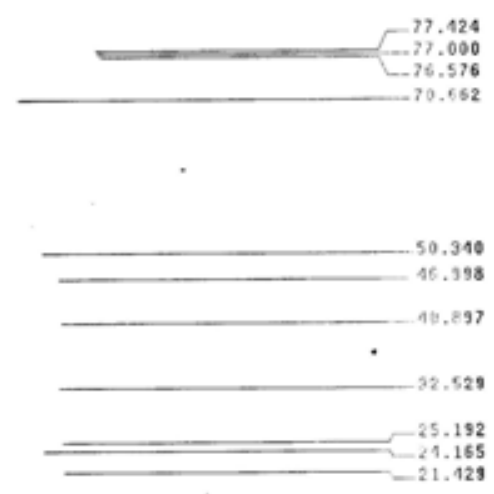

